\title{
DIFFERENTIAL STRUCTURE ON THE DUAL OF A COMPACT LIE GROUP
}

\author{
VÉRONIQUE FISCHER
}

\begin{abstract}
In this paper we define difference operators and homogeneous Sobolev-type spaces on the dual of a compact Lie group. As an application and to show that this defines a relevant differential structure, we state and prove multiplier theorems of Hörmander, Mihlin and Marcinkiewicz types together with the sharpness in the Sobolev exponent for the one of Hörmander type.
\end{abstract}

\section{Contents}

1. Introduction

2. Algebraic structures on $\widehat{G}$

2.1. Preliminaries

2.2. The algebra $\Sigma$

2.3. Difference operators

2.4. Fundamental difference operators

2.5. The structure of differential algebra

2.6. Annihilators of fundamental difference operators

3. Interpretation as Fourier multipliers

3.1. The Peter-Weyl theorem

3.2. Fourier multipliers

3.3. The Laplace-Beltrami operator and its Fourier transform

3.4. Proof of (2) $\Rightarrow$ (11) in Lemma 2.12

4. Homogeneous Sobolev spaces on $\widehat{G}$

4.1. First definition

4.2. First properties of $\dot{H}^{s}(\widehat{G}), s \in \mathbb{N}$

4.3. Definition and properties of $\dot{H}^{s}(\widehat{G}), s \in \mathbb{R}$

4.4. The spaces $\dot{L}_{s}^{\infty}(\widehat{G}: \Sigma)$

4.5. Weak Leibniz estimates

5. Function of $\widehat{\mathcal{L}}$ in $\dot{H}^{s}(\widehat{G})$

5.1. Statements

5.2. Proof of Proposition 5.1

5.3. Proof of Proposition 5.2

Date: June 2018.

2010 Mathematics Subject Classification. 43A22, 43A77, 22E15,12H05.

Key words and phrases. harmonic analysis on compact Lie groups, differential calculus over a noncommutative algebra, homogeneous Sobolev spaces. 
6.1. Historical perspectives 31

6.2. The space $\mathcal{M}_{s}$

6.3. Multiplier theorems on $G \quad 36$

6.4. Proof of Theorem 6.10

6.5. A Marcinkiewicz type condition 39

References

\section{INTRODUCTION}

The notion of differential structure on e.g. smooth manifolds relies on fundamental ideas from geometry, analysis and algebra. It is motivated by questions coming not only from mathematics but also from mathematical and theoretical physics. A modern instance of these ideas and motivations is to consider algebras equipped with operations that generalise differential calculus, the most interesting cases being non-commutative [2]. The first part of this paper is driven by this general quest and proposes an intrinsic differential structure on the dual $\widehat{G}$ of a compact Lie group $G$, namely difference operators and homogeneous Sobolev spaces. The second part is devoted to show how this structure provides the natural framework from an analytical viewpoint.

This structure has already been alluded to in the previous paper of the author [9] where intrinsic difference operators were introduced in order to study the pseudo-differential calculus on the group $G$. The ideas seem to have appeared in the reverse chronological order of what has happened on $\mathbb{R}^{n}$ and on manifolds: there, the standard pseudodifferential theory (on which micro-local and semi-classical analysis relies) emerged in the 60's from the study of singular integrals on $\mathbb{R}^{n}$ (Calderón-Zygmund theory), especially multiplier problems. We refer to Section 6.1 for a (personal) historical viewpoint on multiplier problems.

In the second and last part of this paper, we discuss Fourier multipliers on compact Lie groups with conditions analogue to Marcinkiewicz, Mihlin and Hörmander's. A multiplier symbol $\sigma$ is now a field over the dual $\widehat{G}$ of the group $G$; therefore any two multiplier symbols (and a fortiori operators) may not necessarily commute. The first study in this context goes back to 1971 with Coifman and Weiss' monograph [4] where they developed the CalderónZygmund theory in the setting of spaces of homogeneous types and as an application studied the Fourier multipliers of $S U(2)$, see also [5, 6]. Until the recent investigations of Ruzhansky and Wirth [21], the research into Fourier multipliers on compact Lie groups had been focused on central multipliers [3, 25, 28, 30, 31, 32]. To the author's knowledge, the rest of the literature on the Fourier $L^{p}$-multiplier problem on Lie groups 'in the Hörmander sense' is restricted to the motion group (Rubin in 1976 [19]), to the Heisenberg group stemming from the work of de Michele and Mauceri in 1979 [7], and to the graded nilpotent Lie groups with the recent work of the author with Ruzhansky [10].

Our multiplier theorems will be given in Sections 6.3 and 6.5. More precisely we show a result 'of Hörmander type' and from it, we deduce theorems of Mihlin and of Marcinkiewicz types. The proof is classical and relies on the Calderón-Zygmund theory adapted to the setting of spaces of homogeneous type as in [4]. Our approach uses the differential structures 
on the unitary dual $\widehat{G}$ of the group developed in the first part of the paper, in particular homogeneous Sobolev spaces $\dot{H}^{s}(\widehat{G})$. We reformulate the Hörmander condition using these and show that it is indeed a hypothesis for an $L^{p}$-multiplier theorem, see Sections 6.2 and 6.3 respectively. Furthermore this hypothesis is sharp in the exponent $s$ of the Sobolev space, the critical value being half the topological dimension of the group.

The ideas developed in this paper can be of help in classical problems of harmonic analysis on Lie groups: for example, Marcinkiewicz conditions for products of groups (to obtain generalisation of Marcinkiewicz' [16] $L^{p}$-multiplier result for double trigonometric series still an open problem as formulated in [27, p.63]), case of homogeneous domains such as the real and complex spheres, applications to the study of sub-laplacians, $L^{p}-L^{q}$-boundedness of Fourier symbols under a Lizorkin-type condition, estimates of the $L^{p}-L^{q}$-bounds of particular operators such as spectral projectors. Furthermore, the objects developed in this paper may be of interest in modern non-commutative harmonic analysis (e.g. questions studied in [13]), and also in areas where mathematics and theoretical physics intersect such as quantum Riemannian geometry (see e.g. [2]).

The paper is organised as follows. In Section 2, we define the differential structures on algebras of fields over $\widehat{G}$. In Section $[3$, we recall the interpretation of fields over $\widehat{G}$ as Fourier multipliers. In Section 4 , we define the homogeneous Sobolev spaces on $\widehat{G}$, which allow us to state and prove our multiplier theorems in Section 6. The function of the Laplace-Beltrami operator will be studied in Section 5, and used to state our Hörmander condition and prove its sharpness.

Notation: $\mathbb{N}_{0}=\{0,1,2, \ldots\}$ denotes the set of non-negative integers and $\mathbb{N}=\{1,2, \ldots\}$ the set of positive integers. If $\mathcal{H}_{1}$ and $\mathcal{H}_{2}$ are two Hilbert spaces, we denote by $\mathscr{L}\left(\mathcal{H}_{1}, \mathcal{H}_{2}\right)$ the Banach space of the bounded operators from $\mathcal{H}_{1}$ to $\mathcal{H}_{2}$. If $\mathcal{H}_{1}=\mathcal{H}_{2}=\mathcal{H}$ then we write $\mathscr{L}\left(\mathcal{H}_{1}, \mathcal{H}_{2}\right)=\mathscr{L}(\mathcal{H})$. We may allow ourselves to write $A \asymp B$ when the quantity $A$ and $B$ are equivalent in the sense that there exists a constant such that $C^{-1} A \leq B \leq C A$.

\section{Algebraic structures on $\widehat{G}$}

In this section we develop the differential structure on the unitary dual of a (connected) compact Lie group. After some necessary preliminaries in Section 2.1, we define the difference operators in Section 2.3, and the algebras on which it is defined in Section 2.2. We give a precise definition of the fundamental difference operators in Section 2.4 and we obtain a structure of differential algebra on $\widehat{G}$ in Section 2.5. In the last section, we study the annihilators of the difference operators.

2.1. Preliminaries. In this paper, $G$ always denotes a connected compact Lie group and $n$ is its dimension. Its Lie algebra $\mathfrak{g}$ is the tangent space of $G$ at the neutral element $e_{G}$. We refer to classical textbooks, e.g. [14] and [11] for pedagogical presentationa and proofs of their properties.

The complexification of the real Lie algebra $\mathfrak{g}$ is the complex Lie algebra $\mathfrak{g}_{\mathbb{C}}:=\mathbb{C} \otimes \mathfrak{g}$. We denote by $\mathfrak{U}\left(\mathfrak{g}_{\mathbb{C}}\right)$ its universal enveloping algebra. Having fixed a basis $\left(X_{j}\right)_{j=1}^{n}$ for $\mathfrak{g}$, a basis 
of $\mathfrak{U}\left(\mathfrak{g}_{\mathbb{C}}\right)$ is given by

$$
X^{\alpha}:=X_{1}^{\alpha_{1}} \ldots X_{n}^{\alpha_{n}}, \quad \alpha=\left(\alpha_{1}, \ldots, \alpha_{n}\right) \in \mathbb{N}_{0}^{n},
$$

with the usual convention $X^{0}=\mathrm{I}$. For $d \in \mathbb{N}_{0}$, we also denote by $\mathfrak{U}_{d}\left(\mathfrak{g}_{\mathbb{C}}\right)$ the subspace of $\mathfrak{U}\left(\mathfrak{g}_{\mathbb{C}}\right)$ of elements of degree $\leq d$, that is, the vector space of the complex linear combination of $X^{\alpha},|\alpha| \leq d$.

A representation of $G$ is any continuous group homomorphism $\pi$ from $G$ to the set of automorphisms of a finite dimensional complex space. We will denote this space $\mathcal{H}_{\pi}$. Note that continuity implies smoothness. If $\pi$ is a representation of the group $G$, then we keep the same notation $\pi$ for the corresponding infinitesimal representation of $\mathfrak{g}$ and of $\mathfrak{U}\left(\mathfrak{g}_{\mathbb{C}}\right)$ given via

$$
\pi(X)=\partial_{t=0} \pi\left(\exp _{G}(t X)\right) .
$$

Two representations $\pi_{1}$ and $\pi_{2}$ of $G$ are equivalent when there exists a map $U: \mathcal{H}_{\pi_{1}} \rightarrow \mathcal{H}_{\pi_{2}}$ intertwining the representations, that is, such that $\pi_{2} U=U \pi_{1}$. A representation of $G$ is irreducible when the only sub-spaces invariant under $G$ are trivial. The dual of the group $G$, denoted by $\widehat{G}$, is the set of irreducible representations of $G$ modulo equivalence. We also consider the set $\operatorname{Rep}(G)$ of the equivalence classes of representations modulo equivalence. When possible, we will often identify a representation of $G$ and its class in $\widehat{G}$ or $\operatorname{Rep}(G)$. As a topological set, $\widehat{G}$ is discrete and countable.

The tensor product of two representations $\pi_{1}, \pi_{2} \in \operatorname{Rep}(G)$ is denoted as $\pi_{1} \otimes \pi_{2} \in \operatorname{Rep}(G)$. For $\pi \in \operatorname{Rep}(G)$ and $n \in \mathbb{N}$, we write $\pi^{\otimes n}=\pi \otimes \ldots \otimes \pi \in \operatorname{Rep}(G)$ for the tensor product of $n$ copies of $\pi$. We adopt the convention that $\pi^{\otimes 0}=1_{\widehat{G}}$ is the trivial representation.

2.2. The algebra $\Sigma$. We denote by $\Sigma=\Sigma(G)$ the set of fields of operators over $\widehat{G}$ on the Hilbert space $\oplus_{\pi \in \widehat{G}} \mathcal{H}_{\pi}$ :

$$
\Sigma=\Sigma(G)=\left\{\sigma=\left\{\sigma(\pi) \in \mathscr{L}\left(\mathcal{H}_{\pi}\right): \pi \in \widehat{G}\right\}\right\} .
$$

For a general definition of fields of operators, see e.g. [8, Part II Ch 2]. One checks easily that $\Sigma$ is an algebra (over $\mathbb{C}$ ) for the product of linear mappings.

Since any representation $\pi \in \operatorname{Rep}(G)$ may be written as a finite direct sum $\pi=\oplus_{j} \tau_{j}$ of $\tau_{j} \in \widehat{G}$, any field of operator over $\oplus_{\pi \in \widehat{G}} \mathcal{H}_{\pi}$ may be naturally extended over $\oplus_{\pi \in \operatorname{Rep}(G)} \mathcal{H}_{\pi}$ via $\sigma(\pi):=\oplus_{j} \sigma\left(\tau_{j}\right)$. We will often identify an element $\sigma \in \Sigma$ with its natural extension as a collection over $\operatorname{Rep}(G)$ :

$$
\sigma=\{\sigma(\pi), \pi \in \widehat{G}\} \sim \sigma=\{\sigma(\pi), \pi \in \operatorname{Rep}(G)\} .
$$

Let us recall that the elements of $\mathfrak{U}\left(\mathfrak{g}_{\mathbb{C}}\right)$ also provide elements of $\Sigma$ :

Example 2.1. If $X \in \mathfrak{g}$, then $\mathcal{F}_{G}(X):=\{\pi(X): \pi \in \widehat{G}\} \in \Sigma$. In this case again, the extension to a collection over $\operatorname{Rep}(G)$ is straightforward as it coincides with the definition of $\pi(X)$ for $\pi \in \operatorname{Rep}(G)$, see (2.1).

More generally, for any $Y \in \mathfrak{U}\left(\mathfrak{g}_{\mathbb{C}}\right), \mathcal{F}_{G}(Y):=\{\pi(Y): \pi \in \widehat{G}\} \in \Sigma$. Furthermore, $\mathcal{F}_{G}\left(Y_{1} Y_{2}\right)=\mathcal{F}_{G}\left(Y_{1} Y_{2}\right)$ for any $Y_{1}, Y_{2} \in \mathfrak{U}\left(\mathfrak{g}_{\mathbb{C}}\right)$.

In Section 3.1, we will see more generally that the group Fourier transform $\mathcal{F}_{G}(f)=\widehat{f}$ of a distribution $f \in \mathcal{D}^{\prime}(G)$ defines an element of $\Sigma$. 
2.3. Difference operators. Here we recall and explain further the intrinsic definition of difference operators given in [9, Section 3.2].

For any representation $\tau, \pi \in \operatorname{Rep}(G)$ and $\sigma \in \Sigma(G)$, we define the linear mapping $\Delta_{\tau} \sigma(\pi)$ on $\mathcal{H}_{\tau} \otimes \mathcal{H}_{\pi}$ via:

$$
\Delta_{\tau} \sigma(\pi):=\sigma(\tau \otimes \pi)-\sigma\left(\mathrm{I}_{\tau} \otimes \pi\right) .
$$

Hence $\Delta_{\tau} \sigma$ is a field over $\widehat{G}$ of operators on the Hilbert space $\oplus_{\pi \in \widehat{G}} \mathcal{H}_{\pi} \otimes \mathcal{H}_{\tau}$; note that this Hilbert space may be described as

$$
\oplus_{\pi \in \widehat{G}} \mathcal{H}_{\pi} \otimes \mathcal{H}_{\tau} \simeq L^{2}(G) \otimes \mathcal{H}_{\tau}
$$

or equivalently, the Hilbert space of square-integrable $\mathcal{H}_{\tau}$-valued functions on $G$.

Example 2.2. The dual of the torus $\mathbb{T}=\mathbb{R} / 2 \pi \mathbb{Z}$, is $\widehat{\mathbb{T}}=\left\{e_{\ell}, \ell \in \mathbb{Z}\right\}$ where $e_{\ell}(x)=e^{i \ell x}, x \in \mathbb{T}$. Since $e_{\ell} \otimes e_{m}=e_{\ell+m}$, we see:

$$
\Delta_{e_{\ell}} \sigma\left(e_{m}\right)=\sigma\left(e_{\ell+m}\right)-\sigma\left(e_{m}\right) .
$$

Consequently, identifying $\widehat{\mathbb{T}}$ with the lattice $\mathbb{Z}$, the difference operators are the usual discrete (forward or backward) difference operator.

Example 2.3. Let us consider $\mathrm{I}:=\left\{\mathrm{I}_{\pi}, \pi \in \widehat{G}\right\} \in \Sigma$. Then $\Delta_{\tau} \mathrm{I}=0$ for any $\tau \in \operatorname{Rep}(G)$.

For any $\sigma \in \Sigma$ and $\tau \in \operatorname{Rep} G, \Delta_{\tau} \sigma$ is in $\Sigma_{\tau}$ which is the set defined as follows:

Definition 2.4. For any $\tau \in \operatorname{Rep} G, \Sigma_{\tau}$ denotes the set of fields over $\widehat{G}$ on the Hilbert space $\oplus_{\pi \in \widehat{G}} \mathcal{H}_{\pi} \otimes \mathcal{H}_{\tau}$ :

$$
\Sigma_{\tau}=\left\{\omega=\left\{\omega(\pi) \in \mathcal{H}_{\pi} \otimes \mathcal{H}_{\tau}: \pi \in \widehat{G}\right\}\right\} .
$$

One checks easily that $\Sigma_{\tau}$ is an algebra (over $\mathbb{C}$ ) for the product of linear mappings. Example 2.2 motivates us to observe that if $\tau$ is of dimension 1 , then $\Sigma_{\tau}=\Sigma$.

As for $\Sigma$, we extend by linearity a field $\sigma \in \Sigma_{\tau}$ to a field over $\operatorname{Rep}(G)$, see (2.2) and the preceding paragraph. We now observe that Definition 2.3 also makes sense for fields $\sigma \in \Sigma_{\varphi}$ for some $\varphi \in \operatorname{Rep} G$. Note that the resulting object is then valued in $\Sigma_{\varphi \otimes \tau}$. We have obtained the following definition:

Definition 2.5. For any $\tau, \varphi \in \operatorname{Rep}(G)$, the difference operator $\Delta_{\tau}: \Sigma_{\varphi} \rightarrow \Sigma_{\varphi \otimes \tau}$ is the operator defined via (2.3).

The difference operators $\Delta_{\tau}$ satisfy the following:

Lemma 2.6 (Leibniz property). For any $\sigma_{1}, \sigma_{2} \in \Sigma_{\varphi}(G)$ and $\varphi, \tau, \pi \in \operatorname{Rep}(G)$, we have:

$$
\Delta_{\tau}\left(\sigma_{1} \sigma_{2}\right)(\pi)=\Delta_{\tau}\left(\sigma_{1}\right)(\pi) \sigma_{2}\left(\mathrm{I}_{\tau} \otimes \pi\right)+\sigma_{1}(\tau \otimes \pi) \Delta_{\tau}\left(\sigma_{2}\right)(\pi),
$$

The proof is identical to the one given in [9, Section 4.1]:

Proof. We have:

$$
\begin{aligned}
& \Delta_{\tau}\left(\sigma_{1} \sigma_{2}\right)(\pi)=\left(\sigma_{1} \sigma_{2}\right)(\tau \otimes \pi)-\left(\sigma_{1} \sigma_{2}\right)\left(\mathrm{I}_{\tau} \otimes \pi\right) \\
& \quad=\left(\sigma_{1}(\tau \otimes \pi)-\sigma_{1}\left(\mathrm{I}_{\tau} \otimes \pi\right)\right) \sigma_{2}(\tau \otimes \pi)+\sigma_{1}\left(\mathrm{I}_{\tau} \otimes \pi\right)\left(\sigma_{2}(\tau \otimes \pi)-\sigma_{2}\left(\mathrm{I}_{\tau} \otimes \pi\right)\right) .
\end{aligned}
$$

Thus (2.4) follows. 
Our definition of difference operators allows us to compose difference operators. Naturally, up to the order of tensors, difference operators commute.

Example 2.7. If $\tau, \tau_{1}, \pi \in \operatorname{Rep}(G)$ and $X \in \mathfrak{g}_{\mathbb{C}}$, we have

$$
\Delta_{\tau} \Delta_{\tau_{1}} \mathcal{F}_{G}(X)=0
$$

Proof. We compute easily

$$
\left(\tau_{1} \otimes \pi\right)(X)=\partial_{t=0}\left(\tau_{1} \otimes \pi\right)\left(e^{t X}\right)=\tau_{1}(X) \otimes \mathrm{I}_{\pi}+\mathrm{I}_{\tau_{1}} \otimes \pi(X) .
$$

Hence we obtain readily

$$
\forall \tau_{1}, \pi \in \operatorname{Rep}(G) \quad \forall X \in \mathfrak{g}_{\mathbb{C}} \quad\left(\tau_{1} \otimes \pi\right)(X)=\tau_{1}(X) \otimes \mathrm{I}_{\pi}+\mathrm{I}_{\tau_{1}} \otimes \pi(X) .
$$

Therefore for any $X \in \mathfrak{g}_{\mathbb{C}}$ and $\tau_{1}, \pi \in \operatorname{Rep}(G)$

$$
\begin{aligned}
\Delta_{\tau_{1}} \mathcal{F}_{G}(X)(\pi) & =\mathcal{F}_{G}(X)\left(\tau_{1} \otimes \pi\right)-\mathcal{F}_{G}(X)\left(\mathrm{I}_{\tau_{1}} \otimes \pi\right)=\left(\tau_{1} \otimes \pi\right)(X)-\left(\mathrm{I}_{\tau_{1}} \otimes \pi\right)(X) \\
& =\tau_{1}(X) \otimes \mathrm{I}_{\pi} .
\end{aligned}
$$

Since $\Delta_{\tau_{1}} \mathcal{F}_{G}(X)(\pi)$ is constant in $\pi$, the result follows.

Using Examples 2.3 and 2.7 with the Leibniz property (2.4) recursively, we obtain:

Example 2.8. For any $\beta \in \mathbb{N}_{0}^{n}$, any $\tau_{1}, \ldots, \tau_{m} \in \operatorname{Rep}(G)$ with $m>|\beta|$, and any $\pi \in \operatorname{Rep}(G)$,

$$
\Delta_{\tau_{1}} \ldots \Delta_{\tau_{m}} \mathcal{F}_{G}\left(X^{\beta}\right)=0
$$

Hence $\Delta_{\tau_{1}} \ldots \Delta_{\tau_{m}} \mathcal{F}_{G}(Y)=0$ for any $Y \in \mathfrak{U}_{m-1}\left(\mathfrak{g}_{\mathbb{C}}\right)$.

2.4. Fundamental difference operators. The connected compact Lie group $G$ admits a finite set of fundamental representations:

$$
\operatorname{Fund}(G) \subset \widehat{G} \subset \operatorname{Rep}(G),
$$

in the sense that any representation in $\widehat{G}$ will occur in a tensor products of representations in $\operatorname{Fund}(G)$. See below for a constructive proof of this via the highest weight theory.

Definition $2.9([9])$. The fundamental difference operators are the difference operators

$$
\Delta_{\varphi}, \quad \varphi \in \operatorname{Fund}(G)
$$

corresponding to fundamental representations.

We will denote by $\varphi_{1}, \ldots, \varphi_{f}$ the fundamental representations of $G$ :

$$
\operatorname{Fund}(G)=\left\{\varphi_{1}, \ldots, \varphi_{f}\right\}
$$

For $\alpha \in \mathbb{N}_{0}^{f}$, we will write:

$$
\varphi^{\otimes \alpha}:=\varphi_{1}^{\otimes \alpha_{1}} \otimes \ldots \otimes \varphi_{f}^{\otimes \alpha_{f}} \quad \text { and } \quad \Delta^{\alpha}=\Delta_{\varphi_{1}}^{\alpha_{1}} \ldots \Delta_{\varphi_{f}}^{\alpha_{f}} .
$$


Construction of the fundamental representations. If the compact connected Lie group $G$ is semisimple and simply connected, then its fundamental representations are the ones whose highest weights are the fundamental weights of its Lie algebra. This can be generalised to any compact (connected) Lie group $G$ without further hypotheses in the following way. For background material, we refer to [14] (especially Chapters IV and V) and [11] (especially Section 12). We will also need the following well-known properties and conventions about lattices:

Lemma 2.10. Let $V$ ba a real vector space of finite dimension $n$.

(1) A lattice $\Gamma$ of $V$ is a discrete subgroup of $(V,+)$ or equivalently a set of the form $\oplus_{j=1}^{n} \mathbb{Z} e_{j}$ for a basis $\left(e_{j}\right)_{j=1}^{n}$ of $V$. We may call $\left(e_{j}\right)_{j=1}^{n}$ a basis for $\Gamma$.

(2) If $\Gamma$ is a lattice of $V$, then

$$
\Gamma^{*}:=\left\{\lambda \in V^{*}: \lambda(v) \in \mathbb{Z} \text { for all } v \in \Gamma\right\}
$$

is a lattice of the dual $V^{*}$ of $V$; we may call $\Gamma^{*}$ the dual lattice.

Let us set some notation and convention. Firstly, we consider a compact connected Lie group $G$ of dimension $n$ and centre $Z$. We denote by $\mathfrak{g}$ its Lie algebra and by $\mathfrak{z}$ the centre of its Lie algebra which is also the Lie algebra of $Z$. The derived algebra $\mathfrak{g}_{s s}:=[\mathfrak{g}, \mathfrak{g}]$ of $\mathfrak{g}$ is semi-simple and we have $\mathfrak{g}=\mathfrak{z} \oplus \mathfrak{g}_{s s}$. We denote by $G_{s s}=\exp \mathfrak{g}_{s s}$ the closed connected subgroup of $G$ whose Lie algebra is $\mathfrak{g}_{s s}$. Its centre $Z_{s s}:=Z \cap G_{s s}:=\left\{z_{\ell}, \ell=1, \ldots,\left|Z_{s s}\right|\right\}$ of $G_{s s}$ is finite. The centre of $G$ can be written as the direct product $Z=(Z)_{0} \times Z_{s s}$ where $(Z)_{0}=\exp \mathfrak{z}$ is the connected component of $Z$ at the neutral element.

Secondly, we choose a maximal torus $T$ of $G$. Its Lie algebra $\mathfrak{t}$ decomposes as $\mathfrak{t}=\mathfrak{t}_{\mathfrak{z}} \oplus \mathfrak{t}_{s s}$ where $\mathfrak{t}_{\mathfrak{z}}:=\mathfrak{t} \cap \mathfrak{z}$ and $\mathfrak{t}_{s s}:=\mathfrak{t} \cap \mathfrak{g}_{s s}$. The kernel of the homomorphism $\exp _{G}: \mathfrak{t} \rightarrow T$ between abelian groups is a lattice $\Gamma$ of $\mathfrak{t}$. In general, the lattices $\Gamma \cap \mathfrak{t}_{\mathfrak{z}}$ and $\Gamma \cap \mathfrak{t}_{s s}$ are different from the projections of $\Gamma$ onto $\mathfrak{t}_{\mathfrak{z}}$ and $\mathfrak{t}_{s s}$. Indeed $Z_{s s} \subset T \cap G_{s s}$, so for each $\ell=1, \ldots,\left|Z_{s s}\right|$, there exists $H_{s s, \ell} \in \mathfrak{t}_{s s}, H_{\mathfrak{z}, \ell} \in \mathfrak{t}_{\mathfrak{z}}$ such that $z_{\ell}=\exp H_{s s, \ell}=\exp H_{\mathfrak{z}, \ell}$. Consequently $\left|Z_{s s}\right| H_{s s, \ell} \in \Gamma_{s s}$ and $\left|Z_{s s}\right| H_{\mathfrak{z}, \ell} \in \Gamma_{\mathfrak{z}}$, and we have

$$
\Gamma=\sum_{\ell=1}^{\left|Z_{s s}\right|} \mathbb{Z}\left(H_{s s, \ell}-H_{\mathfrak{z}, \ell}\right)+\Gamma_{s s}+\Gamma_{\mathfrak{z}} .
$$

Thirdly, the set of algebraic elements on $\mathfrak{t}$ is $\Lambda_{0}:=\mathfrak{t}_{\mathfrak{z}}^{*} \bigoplus \bigoplus_{j=1}^{d_{\mathfrak{t}_{s s}}} \mathbb{Z} \omega_{s s, j}$ where $\left(\omega_{s s, j}\right)_{j=1}^{d_{\mathfrak{t}_{s s}}}$ denotes the fundamental weights of the semisimple Lie algebra $\mathfrak{g}_{s s}$ with respect to its Cartan subalgebra $\mathfrak{t}_{s s}$ having fixed an ordering on $\mathfrak{t}_{s s}^{*}$. The analytical integral elements on $\mathfrak{t}$ form the lattice $\Lambda:=\Lambda_{0} \cap \Gamma^{*}$ in $\mathfrak{t}^{*}$. We denote by $\left(\beta_{\ell}\right)_{\ell=1}^{n}$ a basis of $\Lambda$. This induces an ordering of $\mathfrak{t}^{*}$ but also of $\mathfrak{t}_{s s}^{*}$ and of $\mathfrak{t}_{\mathfrak{z}}^{*}$. We may assume that the two orderings on $\mathfrak{t}_{s s}^{*}$ coincide, so that the $\beta_{\ell}$ 's are dominant. Let $\left(\omega_{\mathfrak{z}, k}\right)_{k=1}^{d_{\mathfrak{t} \mathfrak{z}}}$ be a basis of $(\Gamma \cap \mathfrak{z})^{*}$; we may assume that the $\omega_{\mathfrak{z}, k}$ 's are positive for the ordering. Although each $\omega_{\mathfrak{z}, k}$ may not be in $\Gamma^{*}$, we check easily that $\left|Z_{s s}\right| \omega_{\mathfrak{z}, k}$ is in $\Gamma^{*}$ and in fact in $\Lambda$.

Finally, the theorem of the highest weight for compact connected Lie groups states that an irreducible representation on $G$ is characterised up to equivalence by its highest weight which is an analytical integral dominant functional on $\mathfrak{t}$, and that any analytical dominant functional $\lambda$ is the highest weight for an irreducible representation $\pi_{\lambda}$ on $G$. For instance, the 
linear functional $\tilde{\omega}_{\mathfrak{z}}:=\left|Z_{s s}\right| \sum_{k=1}^{d_{\mathfrak{t}}} \omega_{\mathfrak{z}, k}$ is analytical dominant; as it is trivial on $\mathfrak{t}_{s s}$, for any $N \in \mathbb{Z}$, the functional $N \tilde{\omega}_{\mathfrak{z}}$ is also analytical dominant and its corresponding representation $\pi_{N \tilde{\omega}_{\mathfrak{z}}}$ is one-dimensional. If $\lambda$ is a dominant analytical integral functional, then, for an integer $N \in \mathbb{N}_{0}$ large enough, the functional $\tilde{\lambda}:=\lambda+N \tilde{\omega}_{\mathfrak{z}}$ is positive for the ordering and therefore it is written as a linear combination over $\mathbb{N}_{0}$ of $\beta_{\ell}$, i.e. $\tilde{\lambda}=\sum_{\ell=1}^{n} n_{\ell} \beta_{\ell}$ with $n_{\ell} \in \mathbb{N}_{0}$. Since $\tilde{\lambda}$ is also dominant analytical with $\pi_{\tilde{\lambda}}=\pi_{N \tilde{\omega}_{\mathfrak{z}}} \otimes \pi_{\lambda}$, we have $\pi_{\lambda}=\pi_{-N \tilde{\omega}_{\mathfrak{z}}} \otimes \pi_{\tilde{\lambda}}$. Note that $\pi_{\lambda}$ occurs in $\pi_{-\tilde{\omega}_{\mathfrak{\jmath}}}^{\otimes N} \otimes \otimes_{\ell=1}^{n} \pi_{\beta_{\ell}}^{\otimes n_{\ell}}$. Hence we can choose $\pi_{\beta_{1}}, \ldots, \pi_{\beta_{n}}, \bar{\pi}_{-\tilde{\omega}_{\mathfrak{\jmath}}}$ as a set of fundamental representations.

2.5. The structure of differential algebra. In this section, we define a structure of differential algebra for the space of finite linear combinations of elements in $A_{a}:=\bigoplus_{|\alpha|=a} \Sigma_{\varphi \otimes \alpha}$

$$
A:=\sum_{a=0}^{+\infty} A_{a}=\sum_{\alpha \in \mathbb{N}_{0}^{f}}^{\oplus} \Sigma_{\varphi \otimes \alpha} .
$$

The construction is of interest of its own, although we will not use it per se in this paper.

As already noted, for each $\tau \in \operatorname{Rep}(G), \Sigma_{\tau}$ is an algebra over $\mathbb{C}$. Furthermore, for each $\tau, \varphi \in \operatorname{Rep}(G)$, we can define the left and right action of $\Sigma_{\varphi}$ on $\Sigma_{\tau \otimes \varphi}$ via

$$
\left(\sigma_{\varphi} \cdot \sigma_{\tau \otimes \varphi}\right)(\pi)=\left(\mathrm{I}_{\tau} \otimes \sigma_{\varphi}(\pi)\right) \sigma_{\tau \otimes \varphi}(\pi) \quad \text { and } \quad\left(\sigma_{\tau \otimes \varphi} \cdot \sigma_{\varphi}\right)(\pi)=\sigma_{\tau \otimes \varphi}(\pi) \sigma_{\varphi}(\tau \otimes \pi),
$$

for $\sigma_{\varphi} \in \Sigma_{\varphi}, \sigma_{\tau \otimes \varphi} \in \Sigma_{\tau \otimes \varphi}$. Hence $\Sigma_{\tau \otimes \varphi}$ is a bi-module over $\Sigma_{\varphi}$ and the Leibniz formula (2.4) may be reformulated as:

$$
\Delta_{\tau}\left(\sigma_{1}, \sigma_{2}\right)=\left(\Delta_{\tau} \sigma_{1}\right) \cdot \sigma_{2}+\sigma_{1} \cdot\left(\Delta_{\tau} \sigma_{2}\right), \quad \sigma_{1}, \sigma_{2} \in \Sigma_{\varphi}
$$

As each $\Sigma_{\tau}$ is an algebra over $\mathbb{C}$, the space $A$ has a natural structure of algebra over $\mathbb{C}$. Furthermore, the actions defined in the paragraph above equip $A$ with a structure of bi-module over $A_{0}=\Sigma$ as well as of a differential ring with the $\Delta_{\varphi}, \varphi \in \operatorname{Fund}(G)$ as derivations.

2.6. Annihilators of fundamental difference operators. Here, we study the elements of $\Sigma$ which are annihilated by difference operators. More precisely, we obtain the following characterisation:

Proposition 2.11. Let $\sigma \in \Sigma$.

Case $s=1$ : The following are equivalent:

(1) $\Delta_{\varphi} \sigma=0$ for any $\varphi \in \operatorname{Fund}(G)$,

(2) $\Delta_{\varphi} \sigma=0$ for any $\varphi \in \operatorname{Rep}(G)$,

(3) $\sigma=\sigma\left(1_{\widehat{G}}\right) \mathrm{I}$.

Case $s \in \mathbb{N}$ : For any $s \in \mathbb{N}$, the following are equivalent:

(1) $\Delta^{\alpha} \sigma=0$ for all $\alpha \in \mathbb{N}_{0}^{f},|\alpha|=s$,

(2) $\Delta_{\tau_{1}} \ldots \Delta_{\tau_{s}} \sigma=0$ for all $\tau_{1}, \ldots, \tau_{s} \in \operatorname{Rep}(G)$

(3) $\sigma \in \mathcal{F}_{G}\left(\mathfrak{U}_{s-1}\left(\mathfrak{g}_{\mathbb{C}}\right)\right)$.

The case $s=1$ is easily proved: 
Proof of Proposition 2.11, case $s=1$. Let $\sigma \in \Sigma$ be such that $\Delta_{\varphi} \sigma=0$ for any $\varphi \in$ Fund $(G)$. Then

$$
\forall \pi \in \widehat{G}, \quad \forall \varphi \in \operatorname{Fund}(G) \quad \sigma(\varphi \otimes \pi)=\sigma\left(\mathrm{I}_{\varphi} \otimes \pi\right) .
$$

Applying (2.6) to $\pi=1_{\widehat{G}}$, we obtain $\sigma(\varphi)=\sigma\left(1_{\widehat{G}}\right) \mathrm{I}_{\varphi}$ for any $\varphi \in \operatorname{Fund}(G)$. Using this and applying (2.6), we obtain $\sigma\left(\varphi \otimes \varphi^{\prime}\right)=\sigma\left(1_{\widehat{G}}\right) \mathrm{I}_{\varphi \otimes \varphi^{\prime}}$ for any $\varphi, \varphi^{\prime} \in \operatorname{Fund}(G)$. Recursively we obtain that $\sigma$ must be equal to the identity up to the constant $\sigma\left(1_{\widehat{G}}\right)$ at any representation $\pi$ which can be written as a tensor product of fundamental representations, thus by linearity for any $\pi \in \operatorname{Rep}(G)$. Hence $\sigma=\sigma\left(1_{\widehat{G}}\right) \mathrm{I}$. This shows that $(1) \Rightarrow(3)$.

By Example 2.3, $(3) \Rightarrow(2)$. We also have $(2) \Rightarrow(1)$. This shows the case $s=1$ in Proposition 2.11 .

We now turn our attention to second-order fundamental difference operators:

Lemma 2.12. Let $\sigma \in \Sigma$ be such that $\sigma\left(1_{\widehat{G}}\right)=0$. The following properties are equivalent:

(1) $\sigma \in \mathcal{F}_{G}\left(\mathfrak{g}_{\mathbb{C}}\right)$, i.e. there exists $X \in \mathfrak{g}_{\mathbb{C}}$ such that $\sigma=\mathcal{F}_{G}(X)=\{\pi(X), \pi \in \widehat{G}\}$,

(2) for all $\pi_{1}, \pi_{2} \in \operatorname{Rep}(G)$, we have

$$
\sigma\left(\pi_{1} \otimes \pi_{2}\right)=\sigma\left(\pi_{1}\right) \otimes \mathrm{I}_{\pi_{2}}+\mathrm{I}_{\pi_{1}} \otimes \sigma\left(\pi_{2}\right)
$$

(3) the same as (2) with $\pi_{1} \in \operatorname{Fund}(G)$ and $\pi_{2} \in \operatorname{Rep}(G)$,

(4) for all $\varphi, \varphi^{\prime} \in \operatorname{Fund}(G), \Delta_{\varphi} \Delta_{\varphi^{\prime}} \sigma=0$.

We check readily the implications (11) $\Rightarrow(2) \Rightarrow(3)$, and (2) $\Rightarrow$ (41), see Example 2.7, especially (2.5).

Proof of $(\underline{3}) \Rightarrow(2)$. Let $\sigma \in \Sigma$ satisfying $\sigma\left(1_{\widehat{G}}\right)=0$ and Property (3). Let $\varphi, \varphi^{\prime} \in \operatorname{Fund}(G)$ and $\pi \in \operatorname{Rep}(G)$. Property (3) implies

$$
\begin{aligned}
\sigma\left(\varphi \otimes \varphi^{\prime} \otimes \pi\right) & =\sigma(\varphi) \otimes \mathrm{I}_{\varphi^{\prime} \otimes \pi}+\mathrm{I}_{\varphi} \sigma\left(\varphi^{\prime} \otimes \pi\right) \\
& =\sigma(\varphi) \otimes \mathrm{I}_{\varphi^{\prime}} \otimes \mathrm{I}_{\pi}+\mathrm{I}_{\varphi} \otimes \sigma\left(\varphi^{\prime}\right) \otimes \mathrm{I}_{\pi}+\mathrm{I}_{\varphi} \otimes \mathrm{I}_{\varphi^{\prime}} \otimes \sigma(\pi) \\
& =\sigma\left(\varphi \otimes \varphi^{\prime}\right) \otimes \mathrm{I}_{\pi}+\mathrm{I}_{\varphi \otimes \varphi^{\prime}} \otimes \sigma(\pi) .
\end{aligned}
$$

We recognise (2.7) with $\pi_{1}=\varphi \otimes \varphi^{\prime}$. Therefore (2.7) holds for any $\pi_{2} \in \operatorname{Rep}(G)$ and $\pi_{1}$ any fundamental representation or tensor of any two fundamental representations. Proceeding recursively, we see that (2.7) holds for $\pi_{1}$ being any tensors of fundamental representations. Since any representation $\pi_{1} \in \widehat{G}$ occurs in the decomposition into irreducibles of tensor of fundamental representations, this shows that (2.7) holds for any $\pi_{1} \in \widehat{G}$, and thus for any $\pi_{1} \in \operatorname{Rep}(G)$.

Proof of (4) $\Rightarrow(3)$. Let $\sigma \in \Sigma$ satisfying $\sigma\left(1_{\widehat{G}}\right)=0$ and Property (44). Let $\varphi^{\prime} \in \operatorname{Fund}(G)$. Each entry of $\Delta_{\varphi^{\prime}} \sigma$ is an element of $\Sigma$ annihilated by all the difference operators $\Delta_{\varphi}, \varphi \in$ Fund $(G)$, and, by the case $s=1$ which is already proved, is of the form $c$ I for some complex constant $c$. Writing with tensors, this means that there exists $M_{\varphi^{\prime}} \in \mathscr{L}\left(\mathcal{H}_{\varphi^{\prime}}\right)$ such that

$$
\forall \pi \in \operatorname{Rep}(G) \quad \Delta_{\varphi^{\prime}} \sigma(\pi)=M_{\varphi^{\prime}} \otimes \mathrm{I}_{\pi} .
$$

Applying this to $\pi=1_{\widehat{G}}$, we obtain

$$
M_{\varphi^{\prime}}=\Delta_{\varphi^{\prime}} \sigma\left(1_{\widehat{G}}\right)=\sigma\left(\varphi^{\prime} \otimes 1_{\widehat{G}}\right)-\sigma\left(\mathrm{I}_{\tau} \otimes 1_{\widehat{G}}\right)=\sigma\left(\varphi^{\prime}\right)
$$


since $\sigma\left(1_{\widehat{G}}\right)=0$. Therefore $\Delta_{\varphi^{\prime}} \sigma(\pi)=\sigma\left(\varphi^{\prime}\right) \otimes \mathrm{I}_{\pi}$ and Property (3) holds.

Hence Lemma 2.12 will be proved when we show the implication (2) $\Rightarrow(1)$. Let us postpone the proof of this implication until Section 3.4 and show the general case $s \in \mathbb{N}$ recursively. We will need the simple computational remark:

$$
\forall \sigma \in \Sigma, \pi_{1}, \pi_{2}, \pi \in \operatorname{Rep}(G) \quad \Delta_{\pi_{1} \otimes \pi_{2}} \sigma(\pi)=\Delta_{\pi_{1}} \sigma\left(\pi_{2} \otimes \pi\right)+\mathrm{I}_{\pi_{1}} \otimes \Delta_{\pi_{2}} \sigma(\pi) .
$$

which follows from the definition of difference operators.

Proof of Proposition 2.11, general case. First we observe that $(2) \Rightarrow(1)$ and, by Example 2.8, $(3) \Rightarrow(2)$. So it remains to show $(1) \Rightarrow(3)$. Let us prove this recursively on $s$. The equivalences for $s=1,2$ are already proved (or assumed). Let us assume that the equivalences have been established for $s=1,2, \ldots, s_{0}$.

Let $\sigma \in \Sigma$ annihilated by the difference operators $\Delta^{\alpha},|\alpha|=s_{0}+1$. Then by (2.8), it is annihilated by the difference operators $\Delta_{\tau} \Delta^{\alpha}$ for any $\alpha \in \mathbb{N}_{0}^{f},|\alpha|=s_{0}$, and $\tau$ being a fundamental representation or a tensor of two fundamental representations, and recursively any tensor of fundamental representations, therefore any non-trivial representation in fact. Since $\Delta_{1_{\widehat{G}}}$ is zero on $\Sigma$, we can take $\tau$ even trivial. Then for each $\tau \in \operatorname{Rep}(G)$, the $\mathcal{H}_{\tau}$-entries of $\Delta_{\tau} \sigma$ is annihilated by any product of $s_{0}$ fundamental operators and therefore we have:

$$
\Delta_{\tau} \sigma(\pi)=\sum_{|\beta|=s_{0}} M_{\tau, \beta} \otimes \pi(X)^{\beta}+\text { l.o.t. }
$$

where $M_{\tau, \beta} \in \mathscr{L}\left(\mathcal{H}_{\tau}\right)$ and l.o.t. stand for lower order terms, that is here, modulo $\mathscr{L}\left(\mathcal{H}_{\tau}\right) \otimes$ $\pi\left(\mathfrak{U}_{s_{0}-1}\left(\mathfrak{g}_{\mathbb{C}}\right)\right)$. Note that this writing is unique. So for each $\beta \in \mathbb{N}_{0}^{n}$, we have obtained $\left\{M_{\tau, \beta}, \tau \in \operatorname{Rep}(G)\right\} \in \Sigma$ which is 0 at $\tau=1_{\widehat{G}}$.

Furthermore, for any $\tau, \tau^{\prime} \in \operatorname{Rep}(G)$, we have:

$$
\begin{aligned}
\Delta_{\tau \otimes \tau^{\prime}} \sigma(\pi) & =\sum_{|\beta|=s_{0}} M_{\tau \otimes \tau^{\prime}, \beta} \otimes \pi(X)^{\beta}+\text { l.o.t., } \\
\Delta_{\tau} \sigma\left(\tau^{\prime} \otimes \pi\right) & =\sum_{|\beta|=s_{0}} M_{\tau, \beta} \otimes\left(\tau^{\prime} \otimes \pi\right)(X)^{\beta}+\text { l.o.t. } \\
& =\sum_{|\beta|=s_{0}} M_{\tau, \beta} \otimes \mathrm{I}_{\tau^{\prime}} \otimes \pi(X)^{\beta}+\text { l.o.t. }
\end{aligned}
$$

so that $(2.8)$ implies that

$$
\sum_{|\beta|=s_{0}} M_{\tau \otimes \tau^{\prime}, \beta} \otimes \pi(X)^{\beta}=\sum_{|\beta|=s_{0}}\left(M_{\tau, \beta} \otimes \mathrm{I}_{\tau^{\prime}}+\mathrm{I}_{\tau} \otimes M_{\tau^{\prime}, \beta}\right) \otimes \pi(X)^{\beta} .
$$

Hence each field $\left\{M_{\tau, \beta}, \tau \in \operatorname{Rep}(G)\right\}$ satisfies Property (21) of Lemma 2.12. It has to be of the form $\mathcal{F}_{G}\left(Y_{\beta}\right)$ for some $Y_{\beta} \in \mathfrak{g}_{\mathbb{C}}$. Because of (2.9) $, \sigma_{1}:=\sigma-\sum_{|\beta|=s_{0}} \mathcal{F}_{G}\left(Y_{\beta} X^{\beta}\right) \in \Sigma$ is such that $\Delta_{\tau} \sigma$ is of the form (2.9) with $s_{0}$ replaced by $s_{0}-1$. Proceeding as above, we obtain recursively on $s_{0}$ that $\sigma \in \mathcal{F}_{G}\left(\mathfrak{U}_{s_{0}-1}\left(\mathfrak{g}_{\mathbb{C}}\right)\right)$. This concludes the proof of Proposition 2.11.

Proposition 2.11 and Lemma 2.12 are proved once we show the implication (2) $\Rightarrow(11)$ in Lemma 2.12. This will be done in Section 3.4. Our proof will use the interpretation of $\Sigma$ as Fourier multipliers which we now present. 


\section{INTERPRETATION AS FouRIER MULTIPLIERS}

Here we recall the well-known viewpoint on the space $\Sigma$ as the space of symbols for Fourier multiplier operators. This will be an opportunity to set notation and conventions, especially regarding differential operators such as the Laplace-Beltrami operator. References include [27, 15, 3, 5, 6, 4, 7, 25, 28, 30, 32. Eventually, in Section 3.4, we will present the missing part in the proof of Lemma 2.12 ,

3.1. The Peter-Weyl theorem. For any representation $\pi$ of $G$, one can always find an inner product (often denoted by $(\cdot, \cdot)_{\mathcal{H}_{\pi}}$ ) on $\mathcal{H}_{\pi}$ such that the map $\pi(g)$ is unitary on $\mathcal{H}_{\pi}$. If $\pi$ is a representation of $G$, then its coefficients are all the functions of the form

$$
(\pi u, v): x \mapsto(\pi(x) u, v)_{\mathcal{H}_{\pi}}, \quad u, v \in \mathcal{H}_{\pi} .
$$

These are smooth functions on $G$. If a basis $\left\{e_{1}, \ldots, e_{d_{\pi}}\right\}$ of $\mathcal{H}_{\pi}$ is fixed, then the matrix coefficients of $\pi$ are the coefficients $\pi_{i, j}=\left(\pi e_{i}, e_{j}\right)_{\mathcal{H}_{\pi}}, 1 \leq i, j \leq d_{\pi}$. The cofficients of $\pi$ form the finite-dimensional complex vector space

$$
L_{\pi}^{2}(G):=\left\{(\pi u, v), u, v \in \mathcal{H}_{\pi}\right\} .
$$

Note that $L_{\pi}^{2}(G)$ depends only on the equivalence class of $\pi$.

In this paper, we assume that a Haar measure on $G$ has been chosen and that it is normalised to be a probability measure. As is customary, for $p \in[1, \infty)$, we denote by $L^{p}(G)$ the usual Banach space of measurable function $f: G \rightarrow \mathbb{C}$ such that $|f|^{p}$ is integrable.

Theorem 3.1 (Peter-Weyl Theorem). The dual $\widehat{G}$ is discrete and countable. The Hilbert space $L^{2}(G)$ decomposes as the Hilbert direct sum $\oplus_{\pi \in \widehat{G}} L_{\pi}^{2}(G)$. Moreover, if for each $\pi \in \widehat{G}$, one fixes a realisation as a representation with an orthonormal basis of $\mathcal{H}_{\pi}$, then the functions $\sqrt{d}_{\pi} \pi_{i, j}, 1 \leq i, j \leq d_{\pi}, \pi \in \widehat{G}$, form an orthonormal basis of $G$.

If $f \in \mathcal{D}^{\prime}(G)$ is a distribution and $\pi$ is a unitary representation, its group Fourier transform at $\pi$ is denoted by

$$
\pi(f) \equiv \widehat{f}(\pi) \equiv \mathcal{F}_{G} f(\pi) \in \mathscr{L}\left(\mathcal{H}_{\pi}\right)
$$

and defined via

$$
\pi(f)=\int_{G} f(x) \pi(x)^{*} d x, \quad \text { i.e. } \quad(\pi(f) u, v)_{\mathcal{H}_{\pi}}=\int_{G} f(x)(u, \pi(x) v)_{\mathcal{H}_{\pi}} d x
$$

since the coefficient functions are smooth. In particular, we consider the Fourier transform of a function to be defined on $\operatorname{Rep}(G)$ and by restriction on $\widehat{G}$. Note $\widehat{f} \in \Sigma$, see Section 2.2 ,

If $f$ is integrable and $\pi$ unitary, we have

$$
\left\|\mathcal{F}_{G} \kappa(\pi)\right\|_{\mathscr{L}\left(\mathcal{H}_{\pi}\right)} \leq\|\kappa\|_{L^{1}(G)} .
$$

One checks easily that the group Fourier transform maps the convolution of two distributions $f_{1}, f_{2} \in \mathcal{D}^{\prime}(G)$ to the matrix product or composition of their group Fourier transforms:

$$
\mathcal{F}_{G}\left(f_{1} * f_{2}\right)=\widehat{f}_{2} \widehat{f}_{1} .
$$

Recall that the (non-commutative) convolution on $G$ is defined in the usual way:

$$
f * g(x)=\int_{G} f(y) g\left(y^{-1} x\right) d y, \quad f, g \in \mathcal{D}^{\prime}(G) .
$$


The Peter-Weyl theorem yields the Plancherel formula:

$$
\int_{G}|f(x)|^{2} d x=\sum_{\pi \in \widehat{G}} d_{\pi}\|\pi(f)\|_{H S\left(\mathcal{H}_{\pi}\right)}^{2}, \quad f \in L^{2}(G),
$$

and the Fourier inversion formula

$$
f(x)=\sum_{\pi \in \widehat{G}} d_{\pi} \operatorname{Tr}(\pi(x) \pi(f)), \quad f \in \mathcal{D}(G), x \in G .
$$

The finite linear sums of vectors in some $L_{\pi}^{2}(G), \pi \in \widehat{G}$ form the vector space:

$$
L_{\text {fin }}^{2}(G):=\sum_{\pi \in \widehat{G}}^{\perp} L_{\pi}^{2}(G) \text {. }
$$

As each $L_{\pi}^{2}(G)$ is a finite dimensional subspace of $\mathcal{D}(G), L_{\text {fin }}^{2}(G) \subset \mathcal{D}(G)$. The Peter-Weyl Theorem can be stated equivalently as follows: $L_{f i n}^{2}(G)$ is dense in $L^{2}(G)$ and

$$
d_{\pi} \widehat{\pi}\left(\pi^{\prime}\right)=\delta_{\pi=\pi^{\prime}} \mathrm{I}_{\pi^{\prime}}
$$

for any two representations $\pi, \pi^{\prime} \in \widehat{G}$. Here $\mathrm{I}_{\pi^{\prime}}$ is the identity operator on $\mathcal{H}_{\pi^{\prime}}$ and (3.3) means that, when $\pi$ is realised as a matrix representation, we have $d_{\pi} \widehat{\pi}_{i, j}\left(\pi^{\prime}\right)=0$ for any $i, j$ satisfying $1 \leq i \neq j \leq d_{\pi}$, and $d_{\pi} \widehat{\pi}_{i, 1}\left(\pi^{\prime}\right)=1$ for any $i$ satisfying $1 \leq i \leq d_{\pi}$.

3.2. Fourier multipliers. Recall that, on the torus, a Fourier multiplier corresponding to the symbol $\sigma: \mathbb{Z} \rightarrow \mathbb{C}$ is the operator $\mathrm{Op}(\sigma)$ given via

$$
\operatorname{Op}(\sigma) \phi\left(e^{i \theta}\right)=\sum_{\ell=-\infty}^{+\infty} e^{i \ell \theta} \sigma(\ell) \widehat{\phi}(\ell)
$$

where the function $\phi$ admits the Fourier expansion $\phi\left(e^{i \theta}\right)=\sum_{\ell=-\infty}^{+\infty} e^{i \ell \theta} \widehat{\phi}(\ell)$. Clearly $\operatorname{Op}(\sigma)$ is a linear operator well defined on the space of functions that have only a finite number of non-zero Fourier coefficients for instance. Furthermore the Plancherel formula implies that the corresponding multiplier operator admits a unique extension as a linear operator bounded on $L^{2}(\mathbb{T})$ if and only if the symbol is a bounded sequence.

All this is easily generalised to the case of an arbitrary compact Lie group in the following way.

Definition 3.2. We denote by $L^{2}(\widehat{G}: \Sigma)$ the Hilbert space of $\sigma \in \Sigma$ such that

$$
\|\sigma\|_{L^{2}(\widehat{G}: \Sigma)}^{2}:=\sum_{\pi \in \widehat{G}} d_{\pi}\|\sigma(\pi)\|_{H S\left(\mathcal{H}_{\pi}\right)}^{2}
$$

is finite.

The Peter-Weyl theorem (Theorem 3.1) implies that the Hilbert space $L^{2}(\widehat{G}: \Sigma)$ is isometrically isomorphic to $L^{2}(G)$ via the Fourier transform.

Definition 3.3. We denote by $L_{f i n}^{2}(\widehat{G}: \Sigma)$ the space of symbols $\sigma$ such that $\sigma(\pi)=0$ for all $\pi \in \widehat{G}$ but a finite number.

Naturally $L_{\text {fin }}^{2}(\widehat{G}: \Sigma)$ is the subspace of $L^{2}(\widehat{G}: \Sigma)$ isometric to $L_{f i n}^{2}(G)$ via the Fourier transform, see (3.2), and $L_{\text {fin }}^{2}(\widehat{G}: \Sigma)$ is a dense subspace of $L^{2}(\widehat{G}: \Sigma)$. 
Definition 3.4. The Fourier multiplier operator with symbol $\sigma \in \Sigma$ is the operator $\operatorname{Op}(\sigma)$ defined on $L_{\text {fin }}^{2}(G)$ via

$$
\operatorname{Op}(\sigma) \phi(x)=\sum_{\pi \in \widehat{G}} d_{\pi} \operatorname{Tr}(\pi(x) \sigma(\pi) \widehat{\phi}(\pi)), \quad \phi \in L_{f i n}^{2}(G), x \in G .
$$

By definition of $L_{f i n}^{2}(G)$, the summation above is finite and this defines a linear operator $\mathrm{Op}(\sigma): L_{\text {fin }}^{2}(G) \rightarrow L_{\text {fin }}^{2}(G)$ satisfying

$$
\mathcal{F}_{G}\{\mathrm{Op}(\sigma) \phi\}=\sigma \widehat{\phi}
$$

Conversely, if $T$ is a linear operator defined on $L_{\text {fin }}^{2}(G)$ (and with image some complex-valued functions of $x \in G$ ) and if $T$ is invariant under left-translations, then the symbol is recovered via

$$
\sigma(\pi)=\pi(x)^{*}(T \pi)(x), \quad \text { that is, } \quad[\sigma(x, \pi)]_{i, j}=\sum_{k} \overline{\pi_{k i}(x)}\left(T \pi_{k j}\right)(x),
$$

for any $x \in G$, for instance $x=e_{G}$; here one has fixed a matrix realisation of $\pi$ but (3.5) is in fact independent of this realisation. This can be easily checked using (3.3). This shows that the quantisation Op defined above is injective. Moreover (3.5) makes sense for any $\pi \in \operatorname{Rep}(G)$ and one checks easily that this coincides with the natural extension of $\sigma$ to a collection over $\operatorname{Rep}(G)$. In other words, the identification (2.2) may be realised via

$$
\sigma(\pi):=(\operatorname{Op}(\sigma)(\pi))\left(e_{G}\right), \quad \pi \in \operatorname{Rep}(G) .
$$

The considerations above imply that the space $\Sigma$ stands in bijection with the space of Fourier multipliers. For this reason, the elements of $\Sigma$ may be called symbols.

Roughly speaking, any 'reasonable' convolution operator may be viewed as a Fourier multiplier:

Example 3.5. If $\kappa \in \mathcal{D}^{\prime}(G)$, then the convolution operator $T_{\kappa}: \mathcal{D}(G) \rightarrow \mathcal{D}^{\prime}(G), T_{\kappa}(\phi)=\phi * \kappa$ with kernel $\kappa$ is the extension of the Fourier multiplier $\operatorname{Op}(\widehat{\kappa})$ with symbol $\widehat{\kappa}$. Indeed, we have

$$
\widehat{T_{\kappa} \phi}=\widehat{\kappa} \widehat{\phi}, \quad \phi \in \mathcal{D}(G)
$$

Convention: in this paper, we allow ourselves to keep the same notation for a linear operator $T$ with $T: L_{\text {fin }}^{2}(G) \rightarrow L_{\text {fin }}^{2}(G)$ or $T: \mathcal{D}(G) \rightarrow \mathcal{D}^{\prime}(G)$ and any of its possible extension as a continuous operator on a topological spaces of functions on of $G$ as long as such an extension exists and is unique.

Let us give more concrete examples.

Example 3.6. The identity operator on $\mathcal{D}(G)$ is a Fourier multiplier with kernel $\delta_{e_{G}}$ and symbol $\widehat{\delta}_{e_{G}}=$ I. More generally any left-invariant differential operator is a Fourier multiplier since, for any $\beta \in \mathbb{N}_{0}^{n}$, the operator $X^{\beta}$ is a Fourier multiplier with kernel $\left(X^{\beta}\right)^{t} \delta_{e_{G}}$ and symbol

$$
\mathcal{F}_{G}(X)^{\beta}:=\left\{\pi(X)^{\beta}, \pi \in \widehat{G}\right\}
$$


Convention: Here, $\left(X^{\beta}\right)^{t}$ denotes the transpose of $X^{\beta}:\left(X^{\beta}\right)^{t}=(-1)^{|\beta|} X_{n}^{\beta_{n}} \ldots X_{1}^{\beta_{1}}$. We have also used the usual identification of $\mathfrak{g}$ with the space of vector fields on $G$ which are invariant under left translations, and consequently of $\mathfrak{U}\left(\mathfrak{g}_{\mathbb{C}}\right)$ with the Lie algebra of the differential operators on $G$ which are invariant under left translations.

Definition 3.7. We denote by $L^{\infty}(\widehat{G}: \Sigma)$ the Banach space of $\sigma \in \Sigma$ such that

$$
\|\sigma\|_{L^{\infty}(\widehat{G}: \Sigma)}:=\sup _{\pi \in \widehat{G}}\|\sigma(\pi)\|_{\mathscr{L}\left(\mathcal{H}_{\pi}\right)}=\sup _{\pi \in \operatorname{Rep}(G)}\|\sigma(\pi)\|_{\mathscr{L}\left(\mathcal{H}_{\pi}\right)}
$$

is finite.

Recall that if an operator $T \in \mathscr{L}\left(L^{2}(G)\right)$ is left-invariant, that is, invariant under lefttranslation: $T\left(f\left(x_{0} \cdot\right)\right)(x)=(T f)\left(x_{0} x\right), x, x_{0} \in G, f \in L^{2}(G)$, then the Schwartz kernel theorem implies that it is a right convolution operator in the sense that there exists $\kappa \in \mathcal{D}^{\prime}(G)$ such that $T=T_{\kappa}: \phi \mapsto \phi * \kappa$ on $\mathcal{D}(G)$. The Peter-Weyl theorem implies that the Banach space of operators which are left-invariant and bounded on $L^{2}(G)$ is isometric to $L^{\infty}(\widehat{G}: \Sigma)$. Indeed, if $\sigma \in L^{\infty}(\widehat{G}: \Sigma)$, then the corresponding Fourier mulitplier $\operatorname{Op}(\sigma)$ is bounded on $L^{2}(G)$ with operator norm

$$
\|\mathrm{Op}(\sigma)\|_{\mathscr{L}\left(L^{2}(G)\right)}=\|\sigma\|_{L^{\infty}(\widehat{G}: \Sigma)}
$$

The converse holds easily: if $\operatorname{Op}(\sigma)$ is bounded on $L^{2}(G)$ then $\|\sigma\|_{L^{\infty}(\widehat{G}: \Sigma)}$ is finite. Furthermore, Equation (3.7) yields

$$
\left\|T_{\kappa}\right\|_{\mathscr{L}\left(L^{2}(G)\right)}=\sup _{\pi \in \widehat{G}}\left\|\mathcal{F}_{G} \kappa(\pi)\right\|_{\mathscr{L}\left(\mathcal{H}_{\pi}\right)}
$$

3.3. The Laplace-Beltrami operator and its Fourier transform. The (positive) LaplaceBeltrami operator is

$$
\mathcal{L}:=-X_{1}^{2}-\ldots-X_{n}^{2}
$$

where $X_{1}, \ldots, X_{n}$ is an orthonormal basis of $\mathfrak{g}$; here we assume that $\mathfrak{g}$ is equipped with of a scalar product invariant under the adjoint representation of $G$ (this is always possible).

The operator $\mathcal{L}$ does not depend on a particular choice of such a basis. It is invariant under left and right translations and its group Fourier transform is scalar:

$$
\forall \pi \in \widehat{G} \quad \exists ! \lambda_{\pi} \in[0, \infty) \quad \pi(\mathcal{L})=\lambda_{\pi} \mathrm{I}_{\pi} .
$$

We keep the same notation for $\mathcal{L}$ and its self-adjoint extension on $L^{2}(G)$ having as domain of definition the space of all functions $f \in L^{2}(G)$ such that $\mathcal{L} f \in L^{2}(G)$. Then $\mathcal{L}$ is a positive self-adjoint operator on $L^{2}(G)$. The Peter-Weyl Theorem yields an explicit spectral decomposition for $\mathcal{L}$ and of its spectrum $\operatorname{Spec}(\mathcal{L})=\left\{\lambda_{\pi}, \pi \in \widehat{G}\right\}$.

For any function $f:[0, \infty) \rightarrow \mathbb{C}$ the spectral multiplier $f(\mathcal{L})$ is a well defined linear operator on $L_{\text {fin }}^{2}(G)$ with symbol $f(\widehat{\mathcal{L}}):=\left\{f\left(\lambda_{\pi}\right) \mathrm{I}_{\pi}, \pi \in \widehat{G}\right\}$. If $f(\mathcal{L})$ extends to a continuous operator $\mathcal{D}(G) \rightarrow \mathcal{D}^{\prime}(G)$, then by the Schwartz kernel theorem, it admits a distributional convolution kernel which we denote by $f(\mathcal{L}) \delta_{e_{G}} \in \mathcal{D}^{\prime}(G)$ :

$$
f(\mathcal{L}) \phi=\phi *\left(f(\mathcal{L}) \delta_{e_{G}}\right), \quad \phi \in \mathcal{D}(G) .
$$

Naturally the group Fourier transform of this distribution is the symbol of the operator:

$$
\mathcal{F}\left(f(\mathcal{L}) \delta_{e_{G}}\right)(\pi)=f\left(\lambda_{\pi}\right) \mathrm{I}_{\pi}, \quad \pi \in \widehat{G}
$$


The operators $f(\mathcal{L})$ and their kernels have been extensively studied, see e.g. Theorem 5.3 and its proof for a sample of results and references. In the context of our argument, we will study these operators in more details in Section 5. Furthermore, the proof given below of the implication (2) $\Rightarrow(11)$ in Lemma 2.12 will use some properties of $\lambda_{\pi}$ which come from the very rigid structure of weights and roots of compact Lie groups.

3.4. Proof of (2) $\Rightarrow(1)$ in Lemma 2.12, Before starting the proof of the missing implication in Lemma 2.12, let us summarise the properties of the eigenvalues of $\mathcal{L}$ which will be used in the proof. We will use the notion of fundamental representations $\varphi_{1}, \ldots, \varphi_{s}$ explained in Section 2.4 and their corresponding highest weights which we call fundamental weights.

Lemma 3.8. (1) Writing $\omega_{\pi}$ for the highest weight of a representation $\pi \in \widehat{G}$, we have $1+\left|\omega_{\pi}\right| \asymp\left(1+\lambda_{\pi}\right)^{1 / 2}$, in the sense that there exists $C>1$ such that

$$
\forall \pi \in \widehat{G} \quad C^{-1}\left(1+\left|\omega_{\pi}\right|\right) \leq\left(1+\lambda_{\pi}\right)^{1 / 2} \leq C\left(1+\left|\omega_{\pi}\right|\right) .
$$

(2) The highest weight of a representation $\pi \in \widehat{G}$ can be written as a linear combination $\omega_{\pi}=m_{1} \omega_{1}+\ldots+m_{f} \omega_{f}$ of the fundamental weights $\omega_{1}, \ldots, \omega_{f}$, and we have $\sum_{j} m_{j} \asymp$ $1+\left|\omega_{\pi}\right|$ in the sense that there exists $C>1$ such that

$$
\forall \pi \in \widehat{G} \quad C^{-1}\left(1+\left|\omega_{\pi}\right|\right) \leq \sum_{j} m_{j} \leq C\left(1+\left|\omega_{\pi}\right|\right) .
$$

(3) For any $\pi, \rho \in \widehat{G}$, if $\rho \in \widehat{G}$ intervenes in the decomposition of $\tau \otimes \pi$ into irreducibles for some $\tau \in \operatorname{Fund}(G)$ then $\left(1+\lambda_{\rho}\right) \asymp\left(1+\lambda_{\pi}\right)$. Furthermore the supremum over $\pi, \rho \in \widehat{G}$ and $\tau \in \operatorname{Fund}(G)$ such that $\rho \subset \tau \otimes \pi$

$$
\sup \left|\lambda_{\rho}-\lambda_{\pi}\right|<\infty
$$

is finite.

(4) Let $s \in \mathbb{N}$. For any $\pi, \rho \in \widehat{G}$, if $\rho \in \widehat{G}$ occurs in the decomposition of $\tau_{1} \otimes \ldots \otimes \tau_{s} \otimes \pi$ into irreducibles for some $\tau_{1}, \ldots, \tau_{s} \in \operatorname{Fund}(G)$ then $\left(1+\lambda_{\rho}\right) \asymp\left(1+\lambda_{\pi}\right)$. Furthermore the supremum over $\pi, \rho \in \widehat{G}$ and $\tau_{1}, \ldots, \tau_{s} \in \operatorname{Fund}(G)$ such that $\rho \subset \tau_{1} \otimes \ldots \otimes \tau_{s} \otimes \pi$

$$
\sup \left|\lambda_{\rho}-\lambda_{\pi}\right|<\infty
$$

is finite.

Proof of Lemma 3.8. The $\mathcal{L}$-eigenvalue $\lambda_{\pi}$ on $L_{\pi}^{2}(\widehat{G})$ can be written in terms of the weight $\omega_{\pi}$ of $\pi \in \widehat{G}$ as $\lambda_{\pi}=\left|\omega_{\pi}+\delta\right|^{2}-|\delta|^{2}$, where $\delta$ denotes the half sum of the positive roots; see [14, Proposition 5.28] for the semi-simple case, which extends readily to the general case. This implies Part (11) and Part (2) since there are only a finite number of fundamental representations.

Let us prove Part (3). Let $\pi, \rho \in \widehat{G}$ and $\tau \in \operatorname{Fund}(G)$ such that $\rho \in \widehat{G}$ occurs in the decomposition of $\tau \otimes \pi$ into irreducibles. By [14, Proposition 9.72], $\omega_{\rho}=\omega_{\pi}+\mu$ for some weight $\mu$ of $\tau$. The number of fundamental representations and of their weights are finite, so Part (3) follows from Part (11). Part (4) is proved recursively from Part (3).

We can now start the proof of the implication (2) $\Rightarrow(1)$ in Lemma 2.12. Let $\sigma \in \Sigma$ satisfying $\sigma\left(1_{\widehat{G}}\right)=0$ and Property (2) of Lemma 2.12. 
Let us show that $\operatorname{Op}(\sigma): L_{f i n}^{2}(G) \rightarrow \mathcal{D}(G)$ extends uniquely to a continuous linear operator $H^{1}(G) \rightarrow L^{2}(G)$. Let us recall [9, Section 2.3] that the Sobolev spaces $H^{s}(G)$, $s \geq 0$, on $G$ may be defined via local maps and the Euclidean Sobolev spaces on $\mathbb{R}^{n}$, or globally as the closure of $\mathcal{D}(G)$ for the norm:

$$
\|\phi\|_{H^{s}}:=\left\|(\mathrm{I}+\mathcal{L})^{s / 2} \phi\right\|_{L^{2}(G)}=\|\phi\|_{H^{s}} .
$$

Furthermore the space $L_{f i n}^{2}(G)$ is dense in each Hilbert space $H^{s}$ and in the topological vector space $\mathcal{D}(G)=\cap_{s \geq 0} H^{s}=\cap_{s \in \mathbb{N}} H^{s}$. Hence it suffices to show that

$$
\exists C>0 \quad \forall \pi \in \widehat{G} \quad\|\sigma(\pi)\|_{\mathscr{L}\left(\mathcal{H}_{\pi}\right)} \leq C\left(1+\lambda_{\pi}\right)^{1 / 2} .
$$

Let $\pi \in \widehat{G}$. Denoting by $\omega_{j}$ the highest weight of each $\varphi_{j} \in \operatorname{Fund}(G)$, we can write the highest weight of $\pi$ as $\omega_{\pi}=m_{1} \omega_{1}+\ldots+m_{f} \omega_{f}$ and the representation $\pi$ occurs in the decomposition of $\varphi^{\otimes m}$ where $m=\left(m_{1}, \ldots, m_{f}\right) \in \mathbb{N}_{0}^{f}$. Therefore, we have:

$$
\left.\|\sigma(\pi)\|_{\mathscr{L}\left(\mathcal{H}_{\pi}\right)} \leq\left\|\sigma\left(\varphi^{\otimes m}\right)\right\|_{\mathscr{L}\left(\mathcal{H}_{\varphi} \otimes m\right.}\right) .
$$

Applying (2.7) recursively and taking the operator norm yield:

$$
\begin{aligned}
\left\|\sigma\left(\varphi^{\otimes m}\right)\right\|_{\mathscr{L}\left(\mathcal{H}_{\varphi \otimes m}\right)} & \leq m_{1}\left\|\sigma\left(\varphi_{1}\right)\right\|_{\mathscr{L}\left(\mathcal{H}_{\varphi_{1}}\right)}+\ldots+m_{f}\left\|\sigma\left(\varphi_{f}\right)\right\|_{\mathscr{L}\left(\mathcal{H}_{\varphi_{f}}\right)} \\
& \leq \sum_{j} m_{j} \max _{\varphi \in \text { Fund } G}\|\sigma(\varphi)\|_{\mathscr{L}\left(\mathcal{H}_{\varphi}\right) .}
\end{aligned}
$$

By Lemma 3.8 Parts (11) and (21), $\sum_{j} m_{j} \asymp\left(1+\lambda_{\pi}\right)^{1 / 2}$. We have therefore obtained (3.9)), and $\operatorname{Op}(\sigma)$ extends uniquely in a continuous linear operator $H^{1}(G) \rightarrow L^{2}(G)$.

Let us show that

$$
\forall f_{1}, f_{2} \in \mathcal{D}(G) \quad \mathrm{Op}(\sigma)\left(f_{1} f_{2}\right)(x)=\operatorname{Op}(\sigma)\left(f_{1}\right)(x) f_{2}(x)+f_{1}(x) \operatorname{Op}(\sigma)\left(f_{2}\right)(x) .
$$

We have just proved that the linear operator $\operatorname{Op}(\sigma): H^{1}(G) \rightarrow L^{2}(G)$ is continuous. As $L_{\text {fin }}^{2}(G)$ is a dense subspace of $\mathcal{D}(G)$ for the $H^{1}(G)$-norm, it suffices to prove (3.10) for $f_{1}, f_{2} \in L_{f i n}^{2}(G)$, and in fact for $f_{1} \in L_{\pi_{1}}^{2}$ and $f_{2} \in L_{\pi_{2}}^{2}$ for some $\pi_{1}, \pi_{2} \in \widehat{G}$, and furthermore for $f_{1}(x)=\left\langle\pi_{1}(x) v_{1}, w_{1}\right\rangle, f_{2}(x)=\left\langle\pi_{2}(x) v_{2}, w_{2}\right\rangle$ for some unit vector $v_{1}, w_{1} \in \mathcal{H}_{\pi_{1}}, v_{2}, w_{2} \in$ $\mathcal{H}_{\pi_{2}}$. Let us assume that $f_{1}, f_{2}$ are such functions.

The Peter-Weyl theorem (Theorem 3.1) implies readily

$$
\widehat{f}_{1}(\pi): v \longmapsto \delta_{\pi=\pi_{1}} \frac{1}{d_{\pi_{1}}}\left\langle v, v_{1}\right\rangle w_{1}, \quad \text { and } \quad \operatorname{Op}(\sigma)\left(f_{1}\right)(x)=\left\langle\pi_{1}(x) \sigma\left(\pi_{1}\right) w_{1}, v_{1}\right\rangle .
$$

We decompose $\pi_{1} \otimes \pi_{2}=\sum_{\rho} \rho$ into a finite sum of $\rho \in \widehat{G}$. We also decompose the vectors $v_{1} \otimes v_{2}=\sum_{\rho} a_{\rho} v_{\rho}$ and $w_{1} \otimes w_{2}=\sum_{\rho} b_{\rho} w_{\rho}$ with $v_{\rho}, w_{\rho}$ unit vectors of $\mathcal{H}_{\rho}$ and $a_{\rho}, b_{\rho} \in \mathbb{C}$. We have $f_{1} f_{2}=\sum_{\rho} g_{\rho}$ with $g_{\rho}(x)=a_{\rho} b_{\rho}\left\langle\rho(x) v_{\rho}, w_{\rho}\right\rangle$, so

$$
\operatorname{Op}(\sigma)\left(f_{1} f_{2}\right)(x)=\sum_{\rho} \operatorname{Op}(\sigma)\left(g_{\rho}\right)(x)=\sum_{\rho} a_{\rho} b_{\rho}\left\langle\rho(x) \sigma(\rho) w_{\rho}, v_{\rho}\right\rangle,
$$

having used the computations in (3.11) with $g_{\rho}$ instead of $f_{1}$. Using tensor notation, this can be summarised as

$$
\mathrm{Op}(\sigma)\left(f_{1} f_{2}\right)(x)=\left\langle\left(\pi_{1} \otimes \pi_{2}\right)(x) \sigma\left(\pi_{1} \otimes \pi_{2}\right) w_{1} \otimes w_{2}, v_{1} \otimes v_{2}\right\rangle .
$$


We now use $\left(\pi_{1} \otimes \pi_{2}\right)(x)=\pi_{1}(x) \otimes \pi_{2}(x)$ and (2.7) to obtain

$$
\begin{aligned}
\mathrm{Op}(\sigma)\left(f_{1} f_{2}\right)(x)=\langle & \left.\pi_{1}(x) \sigma\left(\pi_{1}\right) w_{1}, v_{1}\right\rangle\left\langle\pi_{2}(x) \sigma\left(\pi_{2}\right) w_{2}, v_{2}\right\rangle \\
& +\left\langle\pi_{1}(x) \sigma\left(\pi_{1}\right) w_{1}, v_{1}\right\rangle\left\langle\pi_{2}(x) \sigma\left(\pi_{2}\right) w_{2}, v_{2}\right\rangle .
\end{aligned}
$$

Thanks to (3.11), we recognise the right-hand side of (3.10) for our choice of functions $f_{1}, f_{2}$ above.

This shows that (3.10) holds for any $f_{1}, f_{2} \in \mathcal{D}(G)$. In other words, $\operatorname{Op}(\sigma)$ is a derivation of $\mathcal{D}(G)$. Therefore, it is a vector field on $G$. As the operator $\operatorname{Op}(\sigma)$ is left-invariant, it must coincide with some left-invariant vector field $X$ identified with an element of $\mathfrak{g}_{\mathbb{C}}$. This shows Property (11). This concludes the proof of Lemma 2.12.

\section{Homogeneous Sobolev spaces on $\widehat{G}$}

In this section, we define homogeneous Sobolev-type spaces on $\widehat{G}$. The motivations behind their definitions is not only their formal appeal but also the fact that they have already appeared indirectly for instance in various works of Coifman and Weiss on $S U(2)$ in the 70's, see Remark 4.5. Beside the definitions, basic properties and characterisations, we discuss weak Leibniz estimates, see Section 4.5. In Section 5, we will discuss the example of functions of $\widehat{\mathcal{L}}$.

4.1. First definition. Before defining the homogeneous Sobolev spaces, we recall [8] that on the space of measurable fields of bounded operators over a standard set one can define naturally two important subspaces: the Banach space (in fact, von Neumann algebra) of fields with bounded essential supremum, and the Banach space (in fact Hilbert space) of square-integrable fields of Hilbert-Schmidt operators. Concretely in the case of $\Sigma_{\tau}$, this leads to the Banach space $L^{\infty}\left(\widehat{G}: \Sigma_{\tau}\right)$ given by the norm

$$
\|\sigma\|_{L^{\infty}\left(\widehat{G}: \Sigma_{\tau}\right)}:=\sup _{\pi \in \widehat{G}}\|\sigma(\pi)\|_{\mathscr{L}\left(\mathcal{H}_{\tau} \otimes \mathcal{H}_{\pi}\right)}, \quad \sigma \in \Sigma_{\tau}
$$

and to the Hilbert space $L^{2}\left(\widehat{G}: \Sigma_{\tau}\right)$ given by the norm

$$
\|\sigma\|_{L^{2}\left(\widehat{G}: \Sigma_{\tau}\right)}^{2}:=\sum_{\pi \in \widehat{G}} d_{\pi}\|\sigma(\pi)\|_{H S\left(\mathcal{H}_{\tau} \otimes \mathcal{H}_{\pi}\right)}^{2} \quad \sigma \in \Sigma_{\tau} .
$$

Naturally, in the case $\tau=1_{\widehat{G}}$, we recover the Banach spaces $L^{\infty}(\widehat{G}: \Sigma)$ and $L^{2}(\widehat{G}: \Sigma)$ defined in Section 3.2 .

Definition 4.1. Let $s \in \mathbb{N}$. The homogeneous Sobolev space $\dot{H}^{s}(\widehat{G})$ on $\widehat{G}$ is the set of $\sigma \in \Sigma$ such that $\Delta^{\alpha} \sigma \in L^{2}\left(\widehat{G}, \Sigma_{\varphi} \otimes \alpha\right)$ for every $\alpha \in \mathbb{N}_{0}^{f},|\alpha|=s$. In this case, the quantity

$$
\|\sigma\|_{\dot{H}^{s}(\widehat{G})}^{2}:=\sum_{|\alpha|=s}\left\|\Delta^{\alpha} \sigma\right\|_{L^{2}\left(\widehat{G}, \Sigma_{\varphi} \otimes \alpha\right.}^{2},
$$

is called the homogeneous Sobolev norm.

Remark 4.2. Note that we will define an equivalent homogeneous Sobolev norm in the case $s=2,3, \ldots$ for which we may use the same notation as in Definition 4.1. See Definition 4.7 and Remark 4.8. 
For instance, the homogeneous Sobolev space $\dot{H}^{1}(\widehat{G})$ on $\widehat{G}$ is the set of symbol $\sigma \in \Sigma$ such that the following quantity is finite:

$$
\|\sigma\|_{\dot{H}^{1}(\widehat{G})}^{2}:=\sum_{\varphi \in \operatorname{Fund}(G)} \sum_{\pi \in \widehat{G}} d_{\pi}\left\|\Delta_{\varphi} \sigma(\pi)\right\|_{H S\left(\mathcal{H}_{\pi} \otimes \mathcal{H}_{\varphi}\right)}^{2} .
$$

The map $\sigma \mapsto\|\sigma\|_{\dot{H}^{1}(\widehat{G})}$ is a semi-norm or in other words a non-definite norm since by Proposition 2.11, we have for any $\sigma \in \Sigma$,

$$
\|\sigma\|_{\dot{H}^{1}(\widehat{G})}=0 \quad \Longleftrightarrow \quad \sigma=\sigma\left(1_{\widehat{G}}\right) \mathrm{I} .
$$

We can define the associated non-definite Hilbert product

$$
\left(\sigma_{1}, \sigma_{2}\right)_{\dot{H}^{1}(\widehat{G})}=\sum_{\varphi \in \operatorname{Fund}(G)} \sum_{\pi \in \widehat{G}} d_{\pi} \operatorname{Tr}_{\mathcal{H}_{\pi} \otimes \mathcal{H}_{\varphi}}\left(\Delta_{\varphi} \sigma_{1}(\pi)\left(\Delta_{\varphi} \sigma_{2}(\pi)\right)^{*}\right) .
$$

More generally, for $s \in \mathbb{N}$, the homogeneous Sobolev space $\dot{H}^{s}(\widehat{G})$ on $\widehat{G}$ is the space of $\sigma \in \Sigma$ such that the semi-norm

$$
\|\sigma\|_{\dot{H}^{s}(\widehat{G})}=\sqrt{\left.\sum_{|\alpha|=s} \sum_{\pi \in \widehat{G}} d_{\pi}\left\|\Delta^{\alpha} \sigma(\pi)\right\|_{H S\left(\mathcal{H}_{\varphi} \otimes \alpha\right.}^{2} \otimes \mathcal{H}_{\pi}\right)}
$$

is finite. By Proposition 2.11 we have for any $\sigma \in \dot{H}^{s}(\widehat{G})$ :

$$
\|\sigma\|_{\dot{H}^{s}(\widehat{G})}=0 \Longleftrightarrow \sigma \in \mathcal{F}_{G}\left(\mathfrak{U}_{s-1}\left(\mathfrak{g}_{\mathbb{C}}\right)\right)
$$

We can define the non-definite Hilbert inner product of $\sigma_{1}, \sigma_{2} \in \dot{H}^{s}(\widehat{G})$ via:

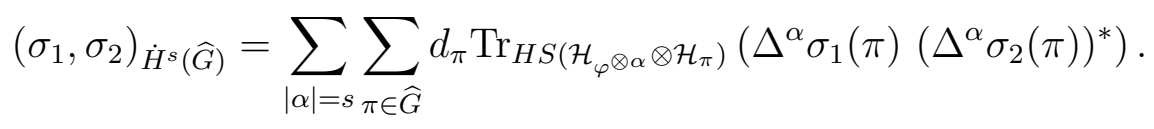

4.2. First properties of $\dot{H}^{s}(\widehat{G}), s \in \mathbb{N}$. This section is devoted to the proof of the following theorem, as well as its consequences:

Theorem 4.3. - Case $s=1$ : The homogeneous Sobolev space $\dot{H}^{1}(\widehat{G})$ quotiented by the kernel $\mathbb{C I}$ of its seminorm is a Hilbert space isometrically isomorphic to $L^{2}\left(q_{1}\right)$, where $q_{1}$ is the function defined via:

$$
q_{1}(x):=\sum_{\varphi \in \operatorname{Fund}(G)}\|\varphi(x)-\mathrm{I}\|_{H S\left(\mathcal{H}_{\varphi}\right)}^{2}=\sum_{\varphi \in \operatorname{Fund}(G)} \sum_{1 \leq i, j \leq d_{\varphi}}\left|[\varphi-\mathrm{I}]_{i, j}(x)\right|^{2} .
$$

- Case $s \in \mathbb{N}:$ More generally, for any $s \in \mathbb{N}$, the homogeneous Sobolev space $\dot{H}^{s}(\widehat{G})$ quotiented by the kernel $\mathcal{F}_{G}\left(\mathfrak{U}_{s-1}\left(\mathfrak{g}_{\mathbb{C}}\right)\right)$ of its seminorm is a Hilbert space isometrically isomorphic to $L^{2}\left(q_{s}\right)$ where

$$
q_{s}(x)=\sum_{|\alpha|=s} \sum_{i, j} \Pi_{\ell=1}^{f}\left|\left[\varphi_{\ell}(x)-\mathrm{I}\right]_{i_{1, \ell}, j_{1, \ell}} \ldots\left[\varphi_{\ell}(x)-\mathrm{I}\right]_{i_{\alpha_{\ell}, \ell}, j_{\alpha_{\ell}, \ell}}\right|^{2},
$$

the sum $\sum_{i, j}$ being a shorthand for $i_{1, \ell}, j_{1, \ell}, \ldots, i_{\alpha_{\ell}, \ell}, j_{\alpha_{\ell}, \ell}$. 
As is customary, for $p \in[1,+\infty)$ and $\omega: G \rightarrow \mathbb{C}$ a non-negative measurable function, $L^{p}(\omega(x) d x)$ denotes the Banach space of measurable function $f: G \rightarrow \mathbb{C}$ such that $\omega(x)|f(x)|^{p}$ is integrable.

The proof of Theorem 4.3 will follow from the statements of this section. Let us first study the function $q_{1}$ and the case $s=1$ :

Proposition 4.4. (1) The function $q_{1}$ is smooth, non-negative, vanishes only at $e_{G}$ and is equivalent to $|x|^{2}$ in the sense that

$$
\exists C>0 \quad \forall x \in G \quad C|x|^{2} \leq q_{1}(x) \leq C^{-1}|x|^{2} .
$$

Consequently, $L^{2}\left(q_{1}\right)=L^{2}\left(|x|^{2} d x\right)$.

(2) The group Fourier transform of a smooth function $f \in \mathcal{D}(G)$ is in $\dot{H}^{1}(\widehat{G})$ and we have:

$$
\|\widehat{f}\|_{\dot{H}^{1}(\widehat{G})}=\|f\|_{L^{2}\left(q_{1}\right)}
$$

(3) The group Fourier transform of a distribution $f \in \mathcal{D}^{\prime}(G)$ is in $\dot{H}^{1}(\widehat{G})$ if and only if $f \in L^{2}\left(|x|^{2}\right)$. Conversely, for every $\sigma \in \dot{H}^{1}(\widehat{G})$ there exists a unique function $f$ locally integrable on $G \backslash\left\{e_{G}\right\}$ satisfying $f \in L^{2}\left(q_{1}\right)$ with $\|f\|_{L^{2}\left(q_{1}\right)}=\|\sigma\|_{\dot{H}^{1}(\widehat{G})}$ and

$$
\forall \phi \in \mathcal{D}(G) \quad(\phi, f)_{L^{2}\left(q_{1}\right)}=(\widehat{\phi}, \sigma)_{\dot{H}^{1}(\widehat{G})} .
$$

The ideas of the proof of Proposition 4.4 Part (1) are essentially the same as in [9, Lemma $5.11]$.

Proof of Proposition 4.4 Part (1). Clearly $q_{1}$ is a non-negative smooth function. As the $q_{i, j}^{(\tau)}$, s vanish at $e_{G}$ so does $q_{1}$. Moreover we have

$$
q_{1}\left(x_{0}\right)=0 \quad \Longleftrightarrow \quad \forall \varphi \in \operatorname{Fund}(G) \quad \varphi\left(x_{0}\right)=\mathrm{I}_{\varphi} \quad \Longleftrightarrow \quad \forall \tau \in \operatorname{Rep}(G) \quad \tau\left(x_{0}\right)=\mathrm{I}_{\tau},
$$

since any representation on $G$ occurs in the decomposition of tensor of fundamental representation. This together with the inversion formula shows that a zero $x_{0}$ of $q_{1}$ must satisfy $f\left(x_{0}\right)=f\left(e_{G}\right)$ for any $f \in \mathcal{D}(G)$, and therefore $x_{0}=e_{G}$. In other words, $e_{G}$ is the only zero of $q_{1}$.

Having fixed an orthonormal basis $\left\{X_{1}, \ldots, X_{n}\right\}$ of $\mathfrak{g}$, we compute easily

$$
X_{k} q_{1}=\sum_{\tau \in \operatorname{Fund}(G)} \sum_{1 \leq i, j \leq d_{\tau}} q_{i, j}^{(\tau)} X_{k} \bar{q}_{i, j}^{(\tau)}+\bar{q}_{i, j}^{(\tau)} X_{k} q_{i, j}^{(\tau)}
$$

so that $X_{k} q_{1}\left(e_{G}\right)=0$, and

$$
X_{\ell} X_{k} q_{1}\left(e_{G}\right)=2 \Re \sum_{\tau \in \operatorname{Fund}(G)} \sum_{1 \leq i, j \leq d_{\tau}} \tau_{i, j}\left(X_{\ell}\right) \bar{\tau}_{i, j}\left(X_{k}\right) .
$$


Let us show that the Hessian matrix $\operatorname{Hess}\left(q_{1}\right)\left(e_{G}\right)=\left[X_{\ell} X_{k} q_{1}\left(e_{G}\right)\right]_{1 \leq \ell, k \leq n}$ is positive definite. If $v=\left(v_{1}, \ldots, v_{n}\right)^{t} \in \mathbb{R}^{n}$, then

$$
\begin{aligned}
\left(\operatorname{Hess}\left(q_{1}\right)\left(e_{G}\right) v\right) \cdot v & =\sum_{1 \leq \ell, k \leq n} X_{\ell} X_{k} q_{1}\left(e_{G}\right) v_{\ell} v_{k} \\
& =2 \Re \sum_{\tau \in \operatorname{Fund}(G)} \sum_{1 \leq i, j \leq d_{\tau}} \sum_{1 \leq \ell, k \leq n} \tau_{i, j}\left(X_{\ell}\right) \bar{\tau}_{i, j}\left(X_{k}\right) v_{\ell} v_{k} \\
& =2 \Re \sum_{\tau \in \operatorname{Fund}(G)} \sum_{1 \leq i, j \leq d_{\tau}}\left|\sum_{1 \leq \ell \leq n} v_{\ell} \tau_{i, j}\left(X_{\ell}\right)\right|^{2} \\
& =\sum_{\tau \in \operatorname{Fund}(G)}\left\|\tau\left(\sum_{1 \leq \ell \leq n} v_{\ell} X_{\ell}\right)\right\|_{H S\left(\mathcal{H}_{\tau}\right)}^{2} .
\end{aligned}
$$

So this quantity is non-negative. Furthermore if it is zero for some $v \in \mathbb{R}^{n}$, then the vector $\sum_{1 \leq \ell \leq n} v_{\ell} X_{\ell} \in \mathfrak{g}$ is in the kernel of every infinitesimal fundamental representation, therefore of any representation of the reductive Lie algebra $\mathfrak{g}$; hence this vector has to be trivial and $v=0$. This shows that $\operatorname{Hess}\left(q_{1}\right)\left(e_{G}\right)$ is positive definite and concludes the proof.

Proof of Proposition 4.4 Part (2). For any $f \in \mathcal{D}(G), \varphi \in \operatorname{Fund}(G)$ and $1 \leq i, j \leq d_{\varphi}$, we observe that $\mathcal{F}_{G}\left([\varphi-\mathrm{I}]_{i, j} f\right)$ is the $i, j$-component of $\Delta_{\varphi} \widehat{f}(\pi)$ in the $\mathcal{H}_{\varphi}$-part of the tensor. So the Plancherel formula implies

$$
\sum_{1 \leq i, j \leq d_{\varphi}}\left\|[\varphi-\mathrm{I}]_{i, j} f\right\|_{L^{2}(G)}^{2}=\sum_{\pi \in \widehat{G}} d_{\pi}\left\|\Delta_{\varphi} \widehat{f}(\pi)\right\|_{H S\left(\mathcal{H}_{\varphi} \otimes \mathcal{H}_{\pi}\right)}^{2} .
$$

Summing over $\varphi \in \operatorname{Fund}(G)$, one obtains $\|f\|_{L^{2}\left(q_{1}\right)}^{2}$ on the left hand-side and $\|\widehat{f}\|_{\dot{H}^{1}(\widehat{G})}$ on the right.

Proof of Proposition 4.4 Part (3). Part (2) implies that the map $\phi \mapsto(\widehat{\phi}, \sigma)_{\dot{H}^{1}(\widehat{G})}$ is linear on $\mathcal{D}(G)$ and satisfies

$$
\left|(\widehat{\phi}, \sigma)_{\dot{H}^{1}(\widehat{G})}\right| \leq\|\widehat{\phi}\|_{\dot{H}^{1}(\widehat{G})}\|\sigma\|_{\dot{H}^{1}(\widehat{G})}=\|\phi\|_{L^{2}\left(q_{1}\right)}\|\sigma\|_{\dot{H}^{1}(\widehat{G})} .
$$

Therefore this linear form extends continuously on the Hilbert space $L^{2}(q)$ and is given by $\phi \mapsto(\phi, f)_{L^{2}\left(q_{1}\right)}$ for a unique $f \in L^{2}\left(q_{1}\right)$. Moreover $\|f\|_{L^{2}\left(q_{1}\right)}$ is equal to the norm of this linear form, which is $\leq\|\sigma\|_{\dot{H}^{1}(\widehat{G})}$. One shows that $\|f\|_{L^{2}\left(q_{1}\right)}=\|\sigma\|_{\dot{H}^{1}(\widehat{G})}$ by considering the sequence of functions $\phi_{\ell}$ such that $\widehat{\phi}_{\ell}(\pi)=\sigma(\pi)$ for $\lambda_{\pi} \leq \ell$ and 0 otherwise and let $\ell \in \mathbb{N}$ tends to $+\infty$. The rest of the proof is given by routine arguments.

Remark 4.5. Note that in the case of $S U(2)$, there is only one fundamental representation $\varphi$; it is the linear action on $\mathbb{C}^{2}$, or in other word $\varphi(g)=g$ for all $g \in S U(2)$. It is then easy to see that the $L^{2}(\widehat{G}: \Sigma)$ norm of the difference operator $\Delta^{2}$ defined in 4, Ch. IV $\S 3$ and Ch. V] applied to a symbol equals the norm $\dot{H}^{1}(\widehat{G})$ of the symbol.

As in the case $s=1$, we prove easily:

Lemma 4.6. Let $s \in \mathbb{N}$.

(1) The function $q_{s}$ is smooth, non-negative, vanishes only at $e_{G}$ and is equivalent to $q_{1}^{s}$ and to $|x|^{2 s}$. Consequently, $L^{2}\left(q_{s}(x) d x\right)=L^{2}\left(q_{1}^{s}(x) d x\right)=L^{2}\left(|x|^{2 s} d x\right)$ for any $s \in \mathbb{R}$. 
(2) For every $f \in \mathcal{D}(G)$, we have:

$$
\|f\|_{L^{2}\left(q_{s}\right)}^{2}=\|\widehat{f}\|_{\dot{H}^{s}(\widehat{G})}^{2} .
$$

(3) For every $\sigma \in \dot{H}^{s}(\widehat{G})$ there exists a unique function $f$ locally integrable on $G \backslash\left\{e_{G}\right\}$ satisfying $f \in L^{2}\left(q_{1}^{s}(x) d x\right)$ with $\|f\|_{L^{2}\left(q_{1}^{s}(x) d x\right)}=\|\sigma\|_{\dot{H}^{s}(\widehat{G})}$ and

$$
\forall \phi \in \mathcal{D}(G) \quad(\phi, f)_{L^{2}\left(q_{1}^{s}(x) d x\right)}=(\widehat{\phi}, \sigma)_{\dot{H}^{s}(\widehat{G})} .
$$

Proof. Part (1) is easily proved. For Part (2), the same arguments as in the proof of Proposition 4.4 Part (2) show:

$$
\sum_{|\alpha|=s} \sum_{i, j} \Pi_{\ell=1}^{f}\left|\left[\varphi_{\ell}(x)-\mathrm{I}\right]_{i_{1, \ell}, j_{1, \ell}} \ldots\left[\varphi_{\ell}(x)-\mathrm{I}\right]_{i_{\alpha_{\ell}, \ell}, j_{\alpha_{\ell}, \ell}}\right|^{2}=\sum_{|\alpha|=s} \sum_{\pi \in \widehat{G}} d_{\pi}\left\|\Delta^{\alpha} \widehat{f}(\pi)\right\|_{H S\left(\mathcal{H}_{\varphi} \otimes \alpha \otimes \mathcal{H}_{\pi}\right)}^{2} .
$$

We recognise $\|f\|_{L^{2}\left(q_{1}\right)}^{2}$ on the left hand-side and $\|\widehat{f}\|_{\dot{H}^{s}(\widehat{G})}$ on the right. We conclude in the same way as in the proof of Proposition 4.4.

This finishes the proof of Theorem 4.3 .

In Parts (3) of Proposition 4.4 and Lemma 4.6, we may abuse the notation and set

$$
f:=\mathcal{F}_{G}^{-1} \sigma \text {. }
$$

This allows us to say that the isometry used in the statement is given by the Fourier transform and its extension.

4.3. Definition and properties of $\dot{H}^{s}(\widehat{G}), s \in \mathbb{R}$. From the results in Section 4.2, we see that the space $\dot{H}^{s}(\widehat{G})$ are growing with $s \in \mathbb{N}$, and that there is a natural extension of the definition for $s \in \mathbb{R}$ :

Definition 4.7. Let $s \in \mathbb{R}$. The homogeneous Sobolev space $\dot{H}^{s}(\widehat{G})$ is defined as

- the set of symbol $\sigma \in L^{2}(\widehat{G}: \Sigma)$ such that $\mathcal{F}_{G}^{-1} \sigma \in L^{2}\left(|x|^{s} d x\right)$ if $s \leq 0$,

- in Definition 4.1 if $s>0$ and $s \in \mathbb{N}$,

- the set of symbol $\sigma \in \dot{H}^{[s]+1}(\widehat{G})$ such that $\mathcal{F}_{G}^{-1} \sigma \in L^{2}\left(|x|^{s} d x\right)$ if $s>0$ and $s \notin \mathbb{N}$.

In all cases, we can define the non-definite norm as

$$
\|\sigma\|_{\dot{H}^{s}(\widehat{G})}:=\left\|\mathcal{F}_{G}^{-1} \sigma\right\|_{L^{2}\left(q_{1}^{s}(x) d x\right)} .
$$

Here $[s]$ denotes the integer part of $s>0$, that is, the largest integer $s_{0} \geq 0$ such that $s_{0} \leq s$.

Remark 4.8. In the cases $s=2,3, \ldots$, in Definitions 4.1 and 4.7, we use the same notation $\|\cdot\|_{\dot{H}^{s}(\widehat{G})}$ for two generally different semi-norms; we allow ourselves this abuse of notation since the two semi-norms are equivalent by Lemma 4.6.

These homogeneous Sobolev spaces $\dot{H}^{s}(\widehat{G})$ enjoy the following properties:

Proposition 4.9. Let $s \in \mathbb{R}$.

(1) The quotient of $\dot{H}^{s}(\widehat{G})$ by the kernel of its norm is a Hilbert space isometrically isomorphic to $L^{2}\left(q_{1}^{s}\right)$, and we have $L^{2}\left(q_{1}^{s}\right)=L^{2}\left(|x|^{2 s} d x\right)$. The kernel of its norm is 0 if $s \leq 0, \mathcal{F}_{G}\left(\mathfrak{U}_{s-1}\left(\mathfrak{g}_{\mathbb{C}}\right)\right)$ if $s \in \mathbb{N}$, and $\mathcal{F}_{G}\left(\mathfrak{U}_{[s]-1}\left(\mathfrak{g}_{\mathbb{C}}\right)\right)$ if $s \in(0,+\infty) \backslash \mathbb{N}$. 
(2) For all $s_{2} \geq s_{1}$, we have $\dot{H}^{s_{2}}(\widehat{G}) \subset \dot{H}^{s_{1}}(\widehat{G})$ and there exists $C>0$ such that

$$
\forall \sigma \in \dot{H}^{s_{2}}(\widehat{G}) \quad\|\sigma\|_{\dot{H}^{s_{1}}(\widehat{G})} \leq C\|\sigma\|_{\dot{H}^{s_{2}}(\widehat{G})} .
$$

(3) If $s<n / 2$ and $\sigma \in \dot{H}^{s}(\widehat{G})$ then $\mathcal{F}_{G}^{-1} \sigma \in L^{1}(G)$ with $\left\|\mathcal{F}_{G}^{-1} \sigma\right\|_{L^{1}(G)} \leq C_{s}\|\sigma\|_{\dot{H}^{s}(\widehat{G})}$.

(4) Let $s_{1}, s_{2} \in \mathbb{R}$. For any $\sigma \in \dot{H}^{s_{1}}(\widehat{G}) \cap \dot{H}^{s_{2}}(\widehat{G})$, we have $\sigma \in \dot{H}^{s}(\widehat{G})$ for any $s \in\left[s_{1}, s_{2}\right]$ and if we write $s$ as $s=\theta s_{1}+(1-\theta) s_{2}$ with $\theta \in[0,1]$ then we have the inequality:

$$
\|\sigma\|_{\dot{H}^{s}(\widehat{G})} \leq\|\sigma\|_{\dot{H}^{s_{1}}(\widehat{G})}^{\theta}\|\sigma\|_{\dot{H}^{s_{2}}(\widehat{G})}^{1-\theta} .
$$

Proof. The properties follow readily from Section 4.1, and

- the inclusion $L^{2}\left(|x|^{2 s_{2}} d x\right) \subset L^{2}\left(|x|^{2 s_{1}} d x\right)$ holds and is continuous when $s_{1} \geq s_{2}$,

- the Cauchy-Schwartz inequality on $G$ implies that $\forall s_{1}, s_{2} \in \mathbb{R}, \theta \in[0,1] \quad\|f\|_{L^{2}\left(|x|^{2\left(\theta s_{1}+(1-\theta) s_{2}\right)} d x\right)} \leq\|f\|_{L^{2}\left(|x|^{2 s_{1}} d x\right)}^{\theta}\|f\|_{L^{2}\left(|x|^{2 s_{2}} d x\right)}^{1-\theta}$, and that

$$
\|f\|_{L^{1}(G)} \leq\left\||\cdot|^{-s}\right\|_{L^{2}} \||\cdot|^{s} f_{L^{2}} .
$$

4.4. The spaces $\dot{L}_{s}^{\infty}(\widehat{G}: \Sigma)$. In this section we define and study subspaces $\dot{L}_{s}^{\infty}(\widehat{G}: \Sigma)$ of $\Sigma$ which will be useful in the study of Leibniz-type estimates in Section 4.5.

Definition 4.10. Let $s \in \mathbb{N}$. The space $\dot{L}_{s}^{\infty}(\widehat{G}: \Sigma)$ is the set of symbol $\sigma \in \Sigma$ such that $\Delta^{\alpha} \sigma \in L^{\infty}\left(\widehat{G}, \Sigma_{\varphi} \otimes \alpha\right)$ for every $\alpha \in \mathbb{N}_{0}^{f},|\alpha|=s$. In this case, we define the quantity

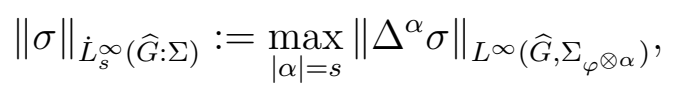

The map $\sigma \mapsto\|\sigma\|_{\dot{L}_{s}^{\infty}(\widehat{G}: \Sigma)}$ is a semi-norm or in other words a non-definite norm since by Proposition 2.11, we have for any $\sigma \in \Sigma$,

$$
\|\sigma\|_{L_{s}^{\infty}(\widehat{G})}=0 \Longleftrightarrow \sigma \in \mathcal{F}_{G}\left(\mathfrak{U}_{s-1}\left(\mathfrak{g}_{\mathbb{C}}\right)\right) .
$$

We define the case $s=0$ as coinciding with $L^{\infty}(\widehat{G}: \Sigma)$ :

$$
\dot{L}_{0}^{\infty}(\widehat{G}: \Sigma):=L^{\infty}(\widehat{G}: \Sigma) \quad \text { and } \quad\|\sigma\|_{\dot{L}_{0}^{\infty}(\widehat{G})}:=\|\sigma\|_{L^{\infty}(\widehat{G}: \Sigma)} .
$$

The following shows that the Fourier transform of integrable functions are in $L_{s}^{\infty}(\widehat{G})$ :

Lemma 4.11. For any $s \in \mathbb{N}_{0}$, there exists $C>0$ such that

$$
\forall \kappa \in L^{1}(G) \quad\|\widehat{\kappa}\|_{\dot{L}_{s}^{\infty}(\widehat{G}: \Sigma)} \leq C\|\kappa\|_{L^{1}\left(|x|^{s} d x\right)} .
$$

Proof. Let $\kappa \in L^{1}(G)$. We have

So

$$
\Delta_{\varphi} \widehat{\kappa}(\pi)=\widehat{\kappa}(\varphi \otimes \pi)-\widehat{\kappa}\left(\mathrm{I}_{\varphi} \otimes \pi\right)=\int_{G} \kappa(x)(\varphi(x)-\mathrm{I})^{*} \otimes \pi(x) d x,
$$

$$
\begin{aligned}
\left\|\Delta_{\varphi} \widehat{\kappa}(\pi)\right\|_{\mathscr{L}\left(\mathcal{H}_{\pi} \otimes \mathcal{H}_{\varphi}\right)} & \leq \int_{G}|\kappa(x)|\left\|(\varphi(x)-\mathrm{I})^{*} \otimes \pi(x)\right\|_{\mathscr{L}\left(\mathcal{H}_{\pi} \otimes \mathcal{H}_{\varphi}\right)} d x \\
& \leq \int_{G}|\kappa(x)|\|\varphi(x)-\mathrm{I}\|_{\mathscr{L}\left(\mathcal{H}_{\varphi}\right)} d x .
\end{aligned}
$$


Now all the operator norms on the finite dimensional space $\mathcal{H}_{\varphi}$ are equivalent, so we may replace the $\mathscr{L}$-operator norm with the Hilbert Schmidt norm:

$$
\|\varphi(x)-\mathrm{I}\|_{\mathscr{L}\left(\mathcal{H}_{\varphi}\right)} \asymp\|\varphi(x)-\mathrm{I}\|_{H S\left(\mathcal{H}_{\varphi}\right)},
$$

so that

$$
\left\|\Delta_{\varphi} \widehat{\kappa}(\pi)\right\|_{\mathscr{L}\left(\mathcal{H}_{\pi} \otimes \mathcal{H}_{\varphi}\right)} \lesssim \int_{G}|\kappa(x)|\|\varphi(x)-\mathrm{I}\|_{H S\left(\mathcal{H}_{\varphi}\right)} d x
$$

Taking the maximum over the finite number of fundamental representations, we obtain:

$$
\|\widehat{\kappa}(\pi)\|_{\dot{L}_{1}^{\infty}(\widehat{G}, \Sigma)} \lesssim \int_{G}|\kappa(x)| \tilde{q}(x) d x
$$

with $\tilde{q}(x):=\max _{\varphi \in \operatorname{Fund}(G)}\|\varphi(x)-\mathrm{I}\|_{H S(\mathcal{H} \varphi)}$. As there are only a finite number of fundamental representations, $\tilde{q}(x) \asymp \sqrt{q_{1}(x)} \asymp|x|$, see Section 4.2. This shows the case $s=1$.

The general case is proved in a similar way.

Lemma 4.12. For any $s_{1}, s_{2} \in \mathbb{N}_{0}$ with $s_{1} \leq s_{2}$ the inclusion $\dot{L}_{s_{1}}^{\infty}(\widehat{G}: \Sigma) \subset \dot{L}_{s_{2}}^{\infty}(\widehat{G})$ holds and there exists $C>0$ such that

$$
\forall \sigma \in \dot{L}_{s_{1}}^{\infty}(\widehat{G}: \Sigma) \quad\|\sigma\|_{\dot{L}_{s_{2}}^{\infty}(\widehat{G})} \leq C\|\sigma\|_{\dot{L}_{s_{1}}^{\infty}(\widehat{G}: \Sigma)} .
$$

Proof. We observe that

$$
\left\|\Delta_{\varphi} \sigma(\pi)\right\|_{\mathscr{L}\left(\mathcal{H}_{\varphi} \otimes \mathcal{H}_{\pi}\right)} \leq\|\sigma(\varphi \otimes \pi)\|_{\mathscr{L}\left(\mathcal{H}_{\varphi} \otimes \mathcal{H}_{\pi}\right)}+\left\|\mathrm{I}_{\varphi} \otimes \sigma(\pi)\right\|_{\mathscr{L}\left(\mathcal{H}_{\varphi} \otimes \mathcal{H}_{\pi}\right)} \leq 2\|\sigma\|_{L^{\infty}(\widehat{G}: \Sigma)} .
$$

This implies the case $s_{1}=0, s_{2}=1$.

The general case is proved using similar considerations.

Thanks to the introduction of $\dot{L}_{s}^{\infty}(\widehat{G}: \Sigma)$, we can now state and prove Leibniz-type estimates.

4.5. Weak Leibniz estimates. Here we collect some results about estimates in homogeneous Sobolev norms of the products of two symbols. We will use the space $\dot{L}_{s}^{\infty}(\widehat{G}: \Sigma)$, $s \in \mathbb{N}_{0}$ defined in Section 4.4. It will be convenient to modify slightly our previous notation for the homogeneous Sobolev spaces

$$
\dot{L}_{s}^{2}(\widehat{G}: \Sigma):=\dot{H}^{s}(\widehat{G}) \text { for any } s \in \mathbb{N}_{0}
$$

Proposition 4.13. For any $s \in \mathbb{N}_{0}$ and $p=2, \infty$, there exists $C=C_{p, s}$ such that for any $\sigma_{1}, \sigma_{2} \in \Sigma$

$$
\left\|\sigma_{1} \sigma_{2}\right\|_{\dot{L}_{s}^{p}(\widehat{G}: \Sigma)} \leq C \sum_{s_{1}+s_{2}=s}\left\|\sigma_{1}\right\|_{\dot{L}_{s_{1}}^{\infty}(\widehat{G}: \Sigma)}\left\|\sigma_{2}\right\|_{\dot{L}_{s_{2}}^{p}(\widehat{G}: \Sigma)},
$$

in the sense that if the right-hand side is finite then the left-hand side is finite and the inequality holds.

Proof. Using the Leibniz-type formula (2.4), we obtain easily with $\|\cdot\|=\|\cdot\|_{H S\left(\mathcal{H}_{\varphi} \otimes \mathcal{H}_{\pi}\right)}$ or $\|\cdot\|=\|\cdot\|_{\mathscr{L}\left(\mathcal{H}_{\varphi} \otimes \mathcal{H}_{\pi}\right)}$ :

$$
\begin{aligned}
\left\|\Delta_{\varphi}\left(\sigma_{1} \sigma_{2}\right)(\pi)\right\| & \leq\left\|\Delta_{\varphi}\left(\sigma_{1}\right)(\pi)\right\|_{\mathscr{L}\left(\mathcal{H}_{\varphi} \otimes \mathcal{H}_{\pi}\right)}\left\|\sigma_{2}\left(\mathrm{I}_{\varphi} \otimes \pi\right)\right\|+\left\|\sigma_{1}(\varphi \otimes \pi)\right\|\left\|_{\mathscr{L}\left(\mathcal{H}_{\varphi} \otimes \mathcal{H}_{\pi}\right)}\right\| \Delta_{\varphi} \sigma_{2}(\pi) \| \\
& \leq\left\|\sigma_{1}\right\|_{\dot{L}_{1}^{\infty}(\widehat{G}: \Sigma)}\left\|\sigma_{2}\left(\mathrm{I}_{\varphi} \otimes \pi\right)\right\|+\left\|\sigma_{1}\right\|_{L^{\infty}(\widehat{G}: \Sigma)}\left\|\Delta_{\varphi} \sigma_{2}(\pi)\right\| .
\end{aligned}
$$


This implies for $p=2$ or $\infty$

$$
\left\|\sigma_{1} \sigma_{2}\right\|_{\dot{L}_{1}^{p}(\widehat{G}: \Sigma)} \lesssim\left\|\sigma_{1}\right\|_{\dot{L}_{1}^{\infty}(\widehat{G}: \Sigma)}\left\|\sigma_{2}\right\|_{L^{p}(\widehat{G}: \Sigma)}+\left\|\sigma_{1}\right\|_{L^{\infty}(\widehat{G}: \Sigma)}\left\|\sigma_{2}\right\|_{\dot{L}_{1}^{p}(\widehat{G}: \Sigma)}
$$

This shows the case $s=1$. The cases $s>1$ are proved in a similar way.

We remark that a different type of Leibniz estimate can be obtained using the isometry of $\dot{H}^{s}$, modulo the kernel of its norm, with $L^{2}\left(q_{2}^{s}(x) d x\right)=L^{2}\left(|x|^{2 s} d x\right)$ and the following lemma. However, this requires to know that the symbols are the Fourier transform of e.g. integrable functions:

Lemma 4.14. Let $s>0$. Then there exists $C_{s}>0$ such that for any two locally integrable functions $f_{1}, f_{2}: G \rightarrow \mathbb{C}$ we have

$$
\left\|f_{1} * f_{2}\right\|_{L^{2}\left(|x|^{2 s} d x\right)} \leq C_{s}\left(\left\|f_{1}\right\|_{L^{2}\left(|x|^{2 s} d x\right)}\left\|f_{2}\right\|_{L^{1}(G)}+\left\|f_{1}\right\|_{L^{2}(G)}\left\|f_{2}\right\|_{L^{1}\left(|x|^{s} d x\right)}\right)
$$

and

$$
\left\|f_{1} * f_{2}\right\|_{L^{2}\left(|x|^{2 s} d x\right)} \leq C_{s}\left(\left\|f_{1}\right\|_{L^{1}(G)}\left\|f_{2}\right\|_{L^{2}\left(|x|^{2 s} d x\right)}+\left\|f_{1}\right\|_{L^{1}\left(|x|^{s} d x\right)}\left\|f_{2}\right\|_{L^{2}(G)}\right) .
$$

Proof. Using the triangle inequality for $|\cdot|$ and the estimate

$$
\forall s>0 \quad \exists C=C_{s}>0 \quad \forall a, b \geq 0 \quad(a+b)^{s} \leq C\left(a^{s}+b^{s}\right),
$$

we obtain

$$
\begin{aligned}
\left\|f_{1} * f_{2}\right\|_{L^{2}\left(|x|^{2 s} d x\right)} & \leq\left\||\cdot|^{s}\left(\left|f_{1}\right| *\left|f_{2}\right|\right)\right\|_{L^{2}(G)} \\
& \leq C\left(\left\|\left(|\cdot|^{s}\left|f_{1}\right|\right) *\left|f_{2}\right|\right\|_{L^{2}(G)}+\left\|\left|f_{1}\right| *\left(|\cdot|^{s}\left|f_{2}\right|\right)\right\|_{L^{2}(G)}\right)
\end{aligned}
$$

and we conclude with Young's convolution inequalities.

In the proof of Lemma 5.5, we will need to estimate the Sobolev norm of $k$-product of the same symbol and obtain an estimate with a precise dependence in $k$. We will use the following technical lemma:

Lemma 4.15. Let $\sigma \in \Sigma$ be such that for any $\varphi \in \operatorname{Fund}(G)$ and any $\pi \in \operatorname{Rep}(G), \sigma(\varphi \otimes \pi)$ commutes with $\sigma\left(\mathrm{I}_{\varphi} \otimes \pi\right)$. Let $k \in \mathbb{N}$.

(1) For any $\varphi \in \operatorname{Fund}(G)$ and any $\pi \in \operatorname{Rep}(G)$, we have

$$
\Delta_{\varphi} \sigma^{k}(\pi)=\Delta_{\varphi} \sigma(\pi) \sum_{j=0}^{k-1} \sigma^{j}(\varphi \otimes \pi) \sigma^{k-1-j}\left(\mathrm{I}_{\varphi} \otimes \pi\right)
$$

(2) For $p=2$ or $\infty$, we have:

$$
\left\|\sigma^{k}\right\|_{\dot{L}_{1}^{p}(\widehat{G}: \Sigma)} \leq\|\sigma\|_{\dot{L}_{1}^{p}(\widehat{G}: \Sigma)} k\|\sigma\|_{\dot{L}^{\infty}(\widehat{G}: \Sigma)}^{k-1} .
$$


(3) Then for $p=2$ or $\infty$, the norm $\left\|\sigma^{k}\right\|_{\dot{L}_{s}^{p}(\widehat{G}: \Sigma)}$ is bounded up to a constant of $s=2,3 \ldots$ by

$$
\begin{aligned}
\|\sigma\|_{\dot{L}_{s}^{p}(\widehat{G}: \Sigma)} k\|\sigma\|_{\dot{L}^{\infty}(\widehat{G}: \Sigma)}^{k-1} & +\|\sigma\|_{\dot{L}_{s-1}^{p}(\widehat{G}: \Sigma)} \sum_{\substack{s_{1}+s_{2}=1 \\
j=0, \ldots, k-1}}\left\|\sigma^{j}\right\|_{\dot{L}_{s_{1}}^{\infty}(\widehat{G}: \Sigma)}\left\|\sigma^{k-1-j}\right\|_{\dot{L}_{s_{2}}^{\infty}(\widehat{G}: \Sigma)}+\ldots \\
& +\|\sigma\|_{\dot{L}_{s-\ell}^{p}(\widehat{G}: \Sigma)} \sum_{\substack{s_{1}+s_{2}=\ell \\
j=0, \ldots, k-1}}\left\|\sigma^{j}\right\|_{\dot{L}_{s_{1}}^{\infty}(\widehat{G}: \Sigma)}\left\|\sigma^{k-1-j}\right\|_{\dot{L}_{s_{2}}^{\infty}(\widehat{G}: \Sigma)}+\ldots \\
& +\|\sigma\|_{\dot{L}_{1}^{p}(\widehat{G}: \Sigma)} \sum_{\substack{s_{1}+s_{2}=s-1 \\
j=0, \ldots, k-1}}\left\|\sigma^{j}\right\|_{\dot{L}_{s_{1}}^{\infty}(\widehat{G}: \Sigma)}\left\|\sigma^{k-1-j}\right\|_{\dot{L}_{s_{2}}^{\infty}(\widehat{G}: \Sigma)} .
\end{aligned}
$$

Proof. Let $\varphi \in \operatorname{Fund}(G)$ and $\pi \in \operatorname{Rep}(G)$. Then

$$
\begin{aligned}
\Delta_{\varphi} \sigma^{k}(\pi) & =\sigma^{k}(\varphi \otimes \pi)-\sigma^{k}\left(\mathrm{I}_{\varphi} \otimes \pi\right) \\
& =\left(\sigma(\varphi \otimes \pi)-\sigma\left(\mathrm{I}_{\varphi} \otimes \pi\right)\right) \sum_{j=0}^{k-1} \sigma^{j}(\varphi \otimes \pi) \sigma^{k-1-j}\left(\mathrm{I}_{\varphi} \otimes \pi\right),
\end{aligned}
$$

and this yields Part (1).

In the formula of Part (1), applying the norm $\|\cdot\|=\|\cdot\|_{H S\left(\mathcal{H}_{\varphi} \otimes \mathcal{H}_{\pi}\right)}$ or $\|\cdot\|=\|\cdot\|_{\mathscr{L}\left(\mathcal{H}_{\varphi} \otimes \mathcal{H}_{\pi}\right)}$, we obtain:

$$
\left\|\Delta_{\varphi} \sigma^{k}(\pi)\right\| \leq\left\|\Delta_{\varphi} \sigma(\pi)\right\|\left\|\sum_{j=0}^{k-1} \sigma^{j}(\varphi \otimes \pi) \sigma^{k-1-j}\left(\mathrm{I}_{\varphi} \otimes \pi\right)\right\|_{\mathscr{L}\left(\mathcal{H}_{\varphi} \otimes \mathcal{H}_{\pi}\right)}
$$

and we easily check that

$$
\left\|\sum_{j=0}^{k-1} \sigma^{j}(\varphi \otimes \pi) \sigma^{k-1-j}\left(\mathrm{I}_{\varphi} \otimes \pi\right)\right\|_{\mathscr{L}\left(\mathcal{H}_{\varphi} \otimes \mathcal{H}_{\pi}\right)} \leq \sum_{j=0}^{k-1}\|\sigma(\varphi \otimes \pi)\|_{\mathscr{L}\left(\mathcal{H}_{\varphi} \otimes \mathcal{H}_{\pi}\right)}^{j}\|\sigma(\pi)\|_{\mathscr{L}\left(\mathcal{H}_{\pi}\right)}^{k-1-j}
$$

is bounded by $k\|\sigma\|_{L^{\infty}(\widehat{G}: \Sigma)}$. This yields Part (2).

Using Part (1) and the Leibniz type formula (2.4) and proceeding as in Part (2) and in the proof of Proposition 4.13, Part (3) follows.

Remark 4.16. The hypotheses on $\sigma \in \Sigma$ in Lemma 4.15, namely that $\sigma(\varphi \otimes \pi)$ commutes with $\sigma\left(\mathrm{I}_{\varphi} \otimes \pi\right)$ for any $\varphi \in \operatorname{Fund}(G)$ and any $\pi \in \operatorname{Rep}(G)$, are satisfied when $\sigma$ is central but also when $\sigma$ is a function of the group Fourier transform of a left-invariant sub-Laplacian on $G$. In any case, they are satisfied when $\sigma$ is a function of the group Fourier transform of the Laplace-beltrami operator on $G$.

\section{Function of $\widehat{\mathcal{L}}$ IN $\dot{H}^{s}(\widehat{G})$}

In this section, we show that certain types of functions of $\widehat{\mathcal{L}}$ are in $\dot{H}^{s}(\widehat{G})$. The methods and ideas presented here are classical, see e.g. [17, Section 1]. 
5.1. Statements. We will give two types of conditions on the functions: a first one of Hörmander type and the second one using Euclidean Sobolev spaces. More precisely, we will show the following two propositions:

Proposition 5.1. Let $G$ be a connected compact Lie group of dimension $n$.

(1) Let $s>0$. There exist $C=C_{s, G}>0$ and $d \in \mathbb{N}_{0}$ such that for any continuous function $f: \mathbb{R} \rightarrow \mathbb{C}$ supported in $[0,1]$ we have

$$
\forall t \in(0,1) \quad\|f(t \widehat{\mathcal{L}})\|_{\dot{H}^{s}(\widehat{G})} \leq C t^{\frac{1}{2}\left(s-\frac{n}{2}\right)} \sup _{\lambda \geq 0, \ell=0, \ldots, d}\left|f^{(\ell)}(\lambda)\right|,
$$

in the sense that if the left-hand side is finite then $f(t \widehat{\mathcal{L}}) \in \dot{H}^{s}(\widehat{G})$ for any $t \in(0,1)$ and the inequality holds.

(2) Let $s>n / 2$. There exist $C=C_{s, G}>0$ and $d \in \mathbb{N}_{0}$ such that for any continuous function $f: \mathbb{R} \rightarrow \mathbb{C}$ we have

$$
\|f(\widehat{\mathcal{L}})\|_{\dot{H}^{s}(\widehat{G})} \leq C \sup _{\lambda \geq 0, \ell=0, \ldots, d}(1+\lambda)^{\ell}\left|f^{(\ell)}(\lambda)\right|,
$$

in the sense that if the left-hand side is finite then $f(\widehat{\mathcal{L}}) \in \dot{H}^{s}(\widehat{G})$ and the inequality holds.

The proof of Proposition 5.1 relies on the properties of the $\dot{H}^{s}(G)$ and of the kernel of $f(t \mathcal{L})$; it is given in Section 5.2 .

Proposition 5.2. Let $G$ be a compact Lie group of dimension n. Let $s^{\prime}>s>\frac{n}{2}$. There exist a constant $C>0$ and a function $\eta \in \mathcal{D}(0, \infty)$ such that for every continuous function $f: \mathbb{R} \rightarrow \mathbb{C}$, we have

$$
\|f(\widehat{\mathcal{L}})\|_{\dot{H}^{s}(\widehat{G})} \leq C \sup _{r>0}\|f(r \cdot) \eta\|_{H^{s^{\prime}(\mathbb{R})}},
$$

in the sense that if the left-hand side is finite then $f(\widehat{\mathcal{L}}) \in \dot{H}^{s}(\widehat{G})$ and the inequality holds.

The proof of Proposition 5.2 will be given in Section 5.3 .

5.2. Proof of Proposition 5.1. Before proving Proposition 5.1, we recall some well known estimates for the heat kernel and other kernels of spectral multipliers of the Laplace-Beltrami operator $\mathcal{L}$ :

Theorem 5.3. (1) For each $t>0$, the heat kernel $p_{t}:=e^{-t \mathcal{L}} \delta_{e_{G}}$ is a positive smooth function on $G$ which satisfies

$$
\forall s, t>0 \quad \int_{G} p_{t}(x) d x=1, \quad p_{t}\left(x^{-1}\right)=p_{t}(x), \quad \text { and } \quad p_{t} * p_{s}=p_{t+s} .
$$

and there exists $C>0$ such that

$$
\forall x \in G, t>0 \quad 0<p_{t}(x) \leq C V(\sqrt{t})^{-1} e^{-\frac{|x|^{2}}{C t}} .
$$

(2) For any $s \geq 0$ and any $\alpha \in \mathbb{N}_{0}^{n}$, there exists a constant $C>0$ such that

$$
\forall t>0 \quad\left\||x|^{s} p_{t}\right\|_{L^{1}(G)} \leq C \sqrt{t}^{s} \quad \text { and } \quad\left\||x|^{s} p_{t}\right\|_{L^{2}(G)} \leq C \sqrt{t}^{s-\frac{n}{2}} .
$$


(3) For any $s \geq 0$, and any $\alpha \in \mathbb{N}_{0}^{n}$, there exists a constant $C>0$ and $d \in \mathbb{N}_{0}$ such that for any $f: \mathbb{R} \rightarrow \mathbb{C}$ continuous and supported in $[0,1]$, we have

$$
\forall t \in(0,1] \quad\left\||x|^{s} X^{\alpha} f(t \mathcal{L}) \delta_{e_{G}}\right\|_{L^{1}(G)} \leq C \sqrt{t}^{s-|\alpha|} \sup _{\substack{\lambda \geq 0 \\ \ell=0, \ldots, d}}\left|f^{(\ell)}(\lambda)\right|,
$$

and

$$
\forall t \in(0,1] \quad\left\||x|^{s} f(t \mathcal{L}) \delta_{e_{G}}\right\|_{L^{2}(G)} \leq C \sqrt{t}^{s-\frac{n}{2}} \sup _{\substack{\lambda \geq 0 \\ \ell=0, \ldots, d}}\left|f^{(\ell)}(\lambda)\right|,
$$

in the sense that if the right-hand sides above are finite, then the left-hand sides are also finite and the inequalities hold.

Above, $|x|=d\left(x, e_{G}\right)$ denotes the Riemannian distance on the Riemann between $x$ and the neutral element $e_{G}$ as it is always possible to define a left-invariant Riemannian distance on $G$, denoted by $d(\cdot, \cdot)$. We also denote by $B(r):=\{|x|<r\}$ the ball about $e_{G}$ of radius $r>0$. The volume $V(r)$ of the ball $B(r)$. It may be estimated via $V(r):=|B(r)| \sim r^{n}$ for $r$ small.

Proof of Theorem 5.3. For Part (1) see [29].

For Part (2), with $p=1,2$, we decompose the integral $\int\left(|x|^{s} p_{t}\right)^{p}$ as $\int_{|x| \leq \sqrt{t}}+\sum_{j=0}^{\infty} \sum_{|x| \sim 2^{j} \sqrt{t}}$. Using the estimates of Part (1) together with $\int_{G} e^{-\frac{|x|^{2}}{C t}} d x \leq C V(\sqrt{t})$, see [29, p.111], we obtain the bound.

Part (3) was proved in [9, Lemma Appendix A.6] for the $L^{1}$-norms using the estimates of the heat kernel and its derivatives which can be deduced from [29]. The methods were classical and mainly due to Alexopoulos, see [1]. They can be easily adapted to the case of the $L^{2}$-norms.

We can now prove Proposition 5.1. Part (1) follows from Proposition 4.9 and Theorem 5.3 Part (3). Let us prove Part (2). We fix a dyadic decomposition, that is, a function $\eta_{0} \in \mathcal{D}(0, \infty)$ valued in $[0,1]$ such that

$$
\sum_{j \in \mathbb{Z}} \eta_{j}=1 \quad \text { on }(0, \infty), \quad \text { where } \eta_{j}(\lambda):=\eta_{0}\left(2^{-j} \lambda\right), \lambda \in \mathbb{R}, j \in \mathbb{Z} .
$$

As the spectrum of $\mathcal{L}$ is a discrete subset of $[0, \infty)$ with no accumulation point, there exists $j_{0}$ depending on $\eta_{0}$ and $G$, such that $\eta_{j}(\widehat{\mathcal{L}})=0$ for all $j<j_{0}$. Furthermore the 0-eigenspace is $\mathbb{C} 1_{G}$, where $1_{G}$ denotes the function on $G$ constantly equal to 1 . Recall $\widehat{1}_{G}=\delta_{1_{\widehat{G}}}$. We have:

$$
f(\widehat{\mathcal{L}})=f(0) \delta_{1_{\widehat{G}}}+\sum_{j \in \mathbb{Z}}\left(f \eta_{j}\right)(\widehat{\mathcal{L}}) .
$$

The sum over $j \in \mathbb{Z}$ is in fact over $j>j_{0}$ and we have

$$
\left\|\delta_{1_{\widehat{G}}}\right\|_{\dot{H}^{s}(\widehat{G})}=\left\|1_{G}\right\|_{L^{2}\left(q_{1}^{s}(x) d x\right)}=\left\|q_{1}^{s}\right\|_{L^{1}(G)}<\infty .
$$

Hence we have obtained

$$
\|f(\widehat{\mathcal{L}})\|_{\dot{H}^{s}(\widehat{G})} \leq|f(0)|\left\|q_{1}^{s}\right\|_{L^{1}(G)}+\sum_{j>j_{0}}\left\|\left(f\left(2^{j} \cdot\right) \eta_{1}\right)\left(2^{-j} \widehat{\mathcal{L}}\right)\right\|_{\dot{H}^{s}(\widehat{G})} .
$$


We estimate each term in the sum using Part (1):

$$
\begin{aligned}
\left\|\left(f \eta_{j}\right)(\widehat{\mathcal{L}})\right\|_{\dot{H}^{s}(\widehat{G})} & =\left\|\left(f\left(2^{j} \cdot\right) \eta_{1}\right)\left(2^{-j} \widehat{\mathcal{L}}\right)\right\|_{\dot{H}^{s}(\widehat{G})} \lesssim 2^{-\frac{j}{2}\left(s-\frac{n}{2}\right)} \sup _{\lambda \geq 0, \ell=0, \ldots, d} \mid \partial_{\lambda}^{\ell}\left\{\left(f\left(2^{j} \lambda\right) \eta_{1}(\lambda)\right\} \mid,\right. \\
& \lesssim 2^{-\frac{j}{2}\left(s-\frac{n}{2}\right)} \sup _{\lambda \geq 0, \ell_{1}=0, \ldots, d}(1+\lambda)^{\ell_{1}}\left|f^{\left(\ell_{1}\right)}(\lambda)\right| .
\end{aligned}
$$

Therefore

$$
\|f(\widehat{\mathcal{L}})\|_{\dot{H}^{s}(\widehat{G})} \lesssim|f(0)|+\sum_{j>j_{0}} 2^{-\frac{j}{2}\left(s-\frac{n}{2}\right)} \sup _{\lambda \geq 0, \ell_{1}=0, \ldots, d}(1+\lambda)^{\ell_{1}}\left|f^{\left(\ell_{1}\right)}(\lambda)\right|,
$$

and the conclusion follows.

5.3. Proof of Proposition 5.2. The main technical point in the proof of Proposition 5.2 is the following estimates:

Lemma 5.4. Let $G$ be a compact Lie group of dimension $n$.

(1) Let $s, s^{\prime} \in \mathbb{R}$ with $s^{\prime}>s+\frac{1}{2}$ and $s \geq 1$. There exists a constant $C>0$ such that for every $f \in H^{s}(\mathbb{R})$ supported in $[0,1]$ and every $t \in(0,1)$ we have

$$
\|f(t \widehat{\mathcal{L}})\|_{\dot{H}^{s}(\widehat{G})} \leq C t^{\frac{1}{2}\left(s-\frac{n}{2}\right)}\|f\|_{H^{s^{\prime}}(\mathbb{R})} .
$$

(2) There exists a constant $C>0$ such that for every function $f: \mathbb{R} \rightarrow \mathbb{C}$ continuous and supported in $[0,1]$, and every $t \in(0,1)$ we have

$$
\|f(t \widehat{\mathcal{L}})\|_{L^{2}(\widehat{G}: \Sigma)} \leq C t^{-\frac{n}{4}} \sup _{\lambda \geq 0}|f(\lambda)| .
$$

Admitting this lemma, the proof of Proposition 5.2 follows easily:

Proof of Proposition 5.2. Proceeding as in the proof of Proposition 5.1 Part (2), we obtain the inequality (5.1). Note that

$$
|f(0)| \leq \sup _{\lambda \geq 0}|f(\lambda)| \lesssim \sup _{r>0}\left\|f(r \cdot) \eta_{1}\right\|_{H^{s_{1}}},
$$

for any $s_{1}>1 / 2$ by the Sobolev embeddings on $\mathbb{R}$.

To estimate each term of the sum in (5.1), we proceed as follows. Thanks to Lemma 5.4, we can interpolate the operator $\phi \mapsto\left(\phi \eta_{1}\right)(t \mathcal{L}) \delta_{e_{G}}$ between $H_{0}^{s^{\prime}}(\Omega)=W_{0}^{s^{\prime}, 2}(\Omega) \rightarrow L^{2}\left(q_{1}^{s}(x) d x\right)$ and $W_{0}^{\epsilon, \infty}(\Omega) \rightarrow L^{2}(d x), \epsilon>0$; here $\Omega$ denotes an open interval of $(0, \infty)$ containing the support of $\eta_{1}$, and the subscript 0 means that the Sobolev spaces are obtained by density of the smooth functions with compact support in $\Omega$. This yields for any $s>s^{\prime}>0$ :

$$
\exists C>0 \quad \forall t \in(0,1) \quad\left\|\left(\phi \eta_{1}\right)(t \widehat{\mathcal{L}})\right\|_{\dot{H}^{s}(\widehat{G})} \lesssim t^{\frac{1}{2}\left(s-\frac{n}{2}\right)}\|\phi\|_{W_{0}^{s^{\prime}, p}(\Omega)},
$$

where $p>2$ is such that $s \geq \theta, s^{\prime}-s \geq \theta \epsilon, \epsilon>\theta / 2$, with $\epsilon \in(0,1)$ arbitrarily small and $\theta:=2 / p$. The Sobolev embeddings gives $\|\phi\|_{W_{0}^{s^{\prime}, p}(\Omega)} \lesssim\|\phi\|_{W_{0}^{\tilde{s}^{\prime}, 2}(\Omega)}$ whenever $\tilde{s}^{\prime}-s^{\prime} \geq \frac{1}{2}-\frac{1}{p}$. Therefore, choosing $p$ large enough, we have obtained:

$$
\forall s^{\prime}>s>0 \quad \forall t \in(0,1) \quad\left\|\left(\phi \eta_{1}\right)(t \widehat{\mathcal{L}})\right\|_{\dot{H}^{s}(\widehat{G})} \lesssim_{s^{\prime}, s} t^{\frac{1}{2}\left(s-\frac{n}{2}\right)}\|\phi\|_{H_{0}^{s^{\prime}}(\Omega)} .
$$


We then apply this to $t=2^{-j}$ and $\phi=f\left(2^{j} \cdot\right) \tilde{\eta}_{1}$, where $\tilde{\eta}_{1} \in \mathcal{D}(\Omega)$ is such that $\tilde{\eta}_{1} \equiv 1$ on the support of $\eta_{1}$. We have therefore obtained for any $s^{\prime}>s>0$ and any $s_{1}>n / 2$ :

$$
\|f(\widehat{\mathcal{L}})\|_{\dot{H}^{s}(\widehat{G})} \lesssim s^{\prime}, s, s_{1} \sup _{r>0}\left\|f(r \cdot) \eta_{1}\right\|_{H^{s_{1}}}+\sum_{j>j_{0}} 2^{-\frac{j}{2}\left(s-\frac{n}{2}\right)}\left\|f\left(2^{j} \cdot\right) \tilde{\eta}_{1}\right\|_{H_{0}^{s^{\prime}}(\Omega)},
$$

and the conclusion follows.

It remains to show Lemma 5.4 .

Proof of Lemma 5.4. Part (2) follows by functional calculus and Theorem 5.3 Part (31). Let us prove Part $(1)$. Let $f \in \mathcal{D}(\mathbb{R})$ supported in $[0,1]$. We write $f(\lambda)$ as $g\left(e^{-\lambda}\right)$ with $g \in H^{s^{\prime}}(\mathbb{R})$ supported in $\left[e^{-1}, 1\right]$. Decomposing $g$ into Fourier series, we have $g(\mu)=\sum_{k \in \mathbb{Z}} a_{k} e^{i k \mu}$ for all $\mu \in(-\pi, \pi)$. Note that

$$
\sum_{k \in \mathbb{Z}}\left|a_{k}\right|^{2}\left(1+k^{2}\right)^{s^{\prime}} \asymp\|g\|_{H^{s^{\prime}}}^{2} \asymp\|f\|_{H^{s^{\prime}}}^{2}
$$

is finite. We see $f(\lambda)=\sum_{k \in \mathbb{Z}} a_{k} e^{i k e^{-\lambda}}$ for any $\lambda \geq 0$, and by functional calculus,

$$
\begin{aligned}
\|f(t \widehat{\mathcal{L}})\|_{\dot{H}^{s}(\widehat{G})} & \leq \sum_{k \in \mathbb{Z}}\left|a_{k}\right|\left\|e^{i k e^{-t \widehat{\mathcal{L}}}}\right\|_{\dot{H}^{s}(\widehat{G})} \\
& \leq\left(\sum_{k \in \mathbb{Z}}\left|a_{k}\right|^{2}\left(1+k^{2}\right)^{s^{\prime}}\right)^{\frac{1}{2}}\left(\sum_{k \in \mathbb{Z}}\left(1+k^{2}\right)^{-s^{\prime}}\left\|e^{i k e^{-t \widehat{\mathcal{L}}}}\right\|_{\dot{H}^{s}(\widehat{G})}^{2}\right)^{\frac{1}{2}},
\end{aligned}
$$

by Cauchy-Schwartz' inequality.

The conclusion follows from (5.2) and Lemma 5.5 below.

Lemma 5.5. Let $G$ be a compact Lie group of dimension $n$. For any $s \geq 1$ there exists $C=C_{s, p}>0$ such that

$$
\forall t \in(0,1), \quad \forall k \in \mathbb{Z} \quad\left\|e^{i k e^{-t \widehat{\mathcal{L}}}}\right\|_{\dot{H}^{s}(\widehat{G})} \leq C \sqrt{t}^{s-\frac{n}{2}}|k|^{s} .
$$

Proof of Lemma 5.5. First let us see that Lemma 5.5 is implied by the following property: for any $s \in \mathbb{N}$ and $p=2, \infty$, there exists $C=C_{s, p}>0$ such that

$$
\forall t \in(0,1), \quad \forall k \in \mathbb{N} \quad\left\|e^{i k e^{-t \widehat{\mathcal{L}}}}\right\|_{\dot{L}_{s}^{p}(\widehat{G}: \Sigma)} \leq C \sqrt{t}^{s-\frac{n}{p}} k^{s} .
$$

By convention, $1 / p=0$ if $p=\infty$. The spaces $\dot{L}_{s}^{p}$ have been defined in Section 4.4.

The case $p=2$ will imply Lemma 5.5 for $s \in \mathbb{N}$ and $k \in \mathbb{N}$. By interpolation (see Proposition 4.9 (4) ), this will imply the cases $s \geq 1$. The case $k=0$ in Lemma 5.5 is trivial, see Example 2.3. The case $k<0$ follows from the case $k>0$ by complex conjugation.

Hence establishing (5.3) will indeed imply Lemma 5.5.

We observe that the symbol $\sigma=e^{i e^{-t \widehat{\mathcal{L}}}}$ satisfies the hypotheses of Lemma 4.15, see Remark 4.16. Using Part (3) of this lemma and assuming that the estimates in (5.3) hold for $k=$ $1,2, \ldots, k_{0}$, we obtain:

$$
\left\|e^{i\left(k_{0}+1\right) e^{-t \widehat{\mathcal{L}}}}\right\|_{\dot{L}_{s}^{p}(\widehat{G}: \Sigma)} \leq C \sqrt{t}^{s-\frac{n}{p}} S,
$$


where

$$
\begin{aligned}
S & :=\sum_{\ell=0}^{s-1} \sum_{s_{1}+s_{2}=\ell} \sum_{j=0}^{k_{0}-1} j^{s_{1}}\left(k_{0}-1-j\right)^{s_{2}} \\
& \leq \sum_{\ell=0}^{s-1} \sum_{s_{1}+s_{2}=\ell} \sum_{j=0}^{k_{0}-1} k_{0}^{\ell+1} \int_{j / k_{0}}^{(j+1) / k_{0}} x^{s_{1}}(1-x)^{s_{2}} d x \\
& \left.\leq \sum_{\substack{s_{1}, s_{2} \in \mathbb{N}_{0} \\
s_{1}+s_{2} \leq s-1}} \int_{0}^{1} x^{s_{1}}(1-x)^{s_{2}} d x\right) \sum_{\ell=0}^{s-1} k_{0}^{\ell+1} \lesssim_{s} k_{0}^{s} ;
\end{aligned}
$$

this is the same estimate in (5.3) for $k_{0}+1$. This will show (5.3) once we have established this estimate for $k=1$; this is what we now do. We want to estimate

$$
\left\|e^{i e^{-t \widehat{\mathcal{L}}}}\right\|_{\dot{L}_{s}^{p}(\widehat{G}: \Sigma)}=\left\|\sum_{\ell=0}^{\infty} \frac{i^{\ell}}{\ell !} e^{-t \ell \widehat{\mathcal{L}}}\right\|_{\dot{L}_{s}^{p}(\widehat{G}: \Sigma)} \leq \sum_{\ell=1}^{\infty} \frac{1}{\ell !}\left\|e^{-t \ell \widehat{\mathcal{L}}}\right\|_{\dot{L}_{s}^{p}(\widehat{G}: \Sigma)} .
$$

By Proposition 4.9 Part (1) and Lemma 4.11, we have

$$
\left\|e^{-t \widehat{\mathcal{L}}}\right\|_{\dot{H}^{s}(\widehat{G})} \asymp\left\|p_{t}\right\|_{L^{2}\left(|x|^{2 s} d x\right)} \quad \text { and } \quad\left\|e^{-t \widehat{\mathcal{L}}}\right\|_{\dot{L}_{s}^{\infty}(\widehat{G}: \Sigma)} \lesssim\left\|p_{t}\right\|_{L^{1}\left(|x|^{s} d x\right)},
$$

so that the estimates on the heat kernel $p_{t}=e^{-t \mathcal{L}} \delta_{0}$ given in Theorem 5.3 yields

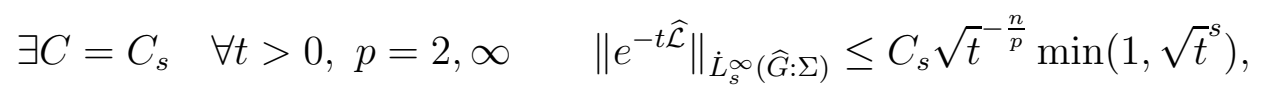

with the convention that $\frac{n}{p}=0$ if $p=\infty$. We use these estimates in

$$
\begin{aligned}
\left\|e^{i e^{-t \widehat{\mathcal{L}}}}\right\|_{\dot{L}_{s}^{p}(\widehat{G}: \Sigma)} & \leq \sum_{\ell=1}^{\infty} \frac{1}{\ell !}\left\|e^{-t \ell \widehat{\mathcal{L}}}\right\|_{\dot{L}_{s}^{p}(\widehat{G}: \Sigma)}=\sum_{\ell: t \ell \leq 1}+\sum_{\ell: t \ell>1} \lesssim \sum_{\ell: t \ell \leq 1} \frac{1}{\ell !}(t \ell)^{\frac{1}{2}\left(s-\frac{n}{p}\right)}+\sum_{\ell: t \ell>1} \frac{1}{\ell !}(t \ell)^{-\frac{n}{2 p}} \\
& \lesssim C_{1} t^{\frac{1}{2}\left(s-\frac{n}{p}\right)}+t^{-\frac{n}{2 p}} \sum_{\ell: t \ell>1} \frac{1}{\ell !},
\end{aligned}
$$

where $C_{1}$ is the finite constant $\sum_{\ell=0}^{\infty} \frac{1}{\ell !} \ell^{\frac{1}{2}\left(s-\frac{n}{p}\right)}$. The last term above is a sum over $\ell>t^{-1}$ which can be viewed as the Taylor remainder of the exponential function on $\mathbb{R}$ between 0 and 1 with order $\left[t^{-1}\right]$. Therefore it is bounded by $e^{1} /\left[t^{-1}\right] ! \lesssim t^{\frac{1}{2}+\frac{1}{t}}$ by the Stirling formula. This implies (5.3) for $k=1$.

This concludes the proofs of (5.3) for any $k \in \mathbb{N}$, thus of Lemma 5.5 .

This concludes the proof of Proposition 5.2 .

\section{Multiplier theOREMS}

In this section we state and prove our multiplier theorems. We start by giving some historical perspectives on the subject in Section 6.1. This leads us to defining the space $\mathcal{M}_{s}$ of symbols of compact Lie groups in Section 6.2. The membership in $\mathcal{M}_{s}$ is a 'Hörmander condition' and we show in Section 6.3 that this implies $L^{p}$-boundedness of the corresponding multiplier operator. We also discuss there the sharpness of the result and a Mihlin-type theorem. The proof of the Hörmander version is given in Section 6.4. A Marcinkiewicz-type 
theorem is discussed in the last section of this paper, recovering as a particular case the historical theorem given at the beginning of the introduction.

6.1. Historical perspectives. The very first multiplier theorem was on the one-dimensional torus $\mathbb{T}$ and its statement (see theorem below) strikingly suggests the use of discrete derivatives and differential structure on the dual of $\mathbb{T}$ :

Theorem 6.1. [Marcinkiewicz multiplier theorem (1939) [16]] If $\sigma: \mathbb{Z} \rightarrow \mathbb{C}$ is a sequence such that the suprema

$$
\sup _{\ell}|\sigma(\ell)|<\infty \quad \text { and } \quad \sup _{j \in \mathbb{N}_{0}} \sum_{2^{j} \leq \ell^{2} \leq 2^{j+1}}|\sigma(\ell)-\sigma(\ell+1)|
$$

are finite, then the corresponding Fourier multiplier is bounded on $L^{p}(\mathbb{T}), 1<p<\infty$.

The next known result on Fourier multipliers is on $\mathbb{R}^{n}$ :

Theorem 6.2 (Mihlin's multiplier theorem (1956) [18]). If a function $\sigma$ defined on $\mathbb{R}^{n} \backslash\{0\}$ has at least $[n / 2]+1$ continuous derivatives that satisfy

$$
\forall \alpha \in \mathbb{N}_{0}^{n},|\alpha| \leq[n / 2]+1, \quad\left|\partial^{\alpha} \sigma(\xi)\right| \leq C_{\alpha}|\xi|^{-|\alpha|},
$$

(where $[t]$ is the integer part of $t$ ), then the Fourier multiplier operator $\operatorname{Op}(\sigma)$ associated with $\sigma$, initially defined on Schwartz functions via

$$
\operatorname{Op}(\sigma) \phi:=\mathcal{F}^{-1}\{\sigma \widehat{\phi}\}
$$

(where $\mathcal{F} \phi=\widehat{\phi}$ denotes the Euclidean Fourier transform) admits a bounded extension on $L^{p}\left(\mathbb{R}^{n}\right)$ for all $1<p<\infty$; for $p=1, \operatorname{Op}(\sigma)$ is bounded from $L^{1}$ to weak- $L^{1}$.

Shortly afterwards, Hörmander gave a further generalisation and simplification of Mihlin's results:

Theorem 6.3 (Hormander's multiplier theorem (1960)). If $\sigma$ is locally uniformly in a Sobolev space $H^{s}\left(\mathbb{R}^{n}\right)$ for some $s>n / 2$, that is, requiring that the quantity

$$
\|\sigma\|_{l . u . H^{s}\left(\mathbb{R}^{n}\right), \eta}:=\sup _{r>0}\|\sigma(r \cdot) \eta\|_{H^{s}\left(\mathbb{R}^{n}\right)}
$$

is finite for some non-zero smooth function $\eta \in \mathcal{D}(0, \infty)$, then the Fourier multiplier operator $\operatorname{Op}(\sigma)$ admits a bounded extension on $L^{p}\left(\mathbb{R}^{n}\right)$ for all $1<p<\infty$; for $p=1, \operatorname{Op}(\sigma)$ is bounded from $L^{1}$ to weak- $L^{1}$.

Note that the proof only requires a supremum over $r=2^{j / 2}, j \in \mathbb{Z}$.

Multipliers problems have been extensively studied in various contexts. In his textbook on singular integrals [26], E. Stein presented results and related problems in the Euclidean context. Fourier multipliers have been explored in completely abstract functional settings, see the 1971 textbook by Larsen [15]. However in this paper we restrict our attention to MihlinHörmander type theorems. On any compact manifold, general (and often sharp) results for Fourier integral or pseudo-differential operators have been known for nearly thirty years, see e.g. [23, 22]. In the context of Lie groups, the focus of the problem 'in the Hörmander sense' has been on spectral multipliers in operators such as sub-Laplacians: see for instance [1, 17, 22, 24, 27] to cite only the results or methods mentioned in this paper. The list 
of references (known to the author at the time of writing) on Fourier multipliers 'in the Hörmander sense' is much shorter: for compact Lie groups [4, 5, 6, 21] and [3, 25, 28, 30, 31, 32] with an emphasis on central multipliers, and for non-compact Lie groups [19, 7, 10], see the discussion of these matters in the introduction (Section 11).

The multiplier problems continue to be an active subject of research in non-commutative harmonic and functional analysis, see e.g. [13].

The Hörmander condition. It is a routine exercise to check that the quantity in (6.3) is finite independently of a choice of $\eta$, in the sense that if $\eta_{1}, \eta_{2} \in \mathcal{D}(0, \infty)$ are non-zero, then $\|\sigma\|_{l . u . H^{s}\left(\mathbb{R}^{n}\right), \eta_{1}} \asymp\|\sigma\|_{l . u . H^{s}\left(\mathbb{R}^{n}\right), \eta_{2}}$. The Sobolev embeddings imply that for $s>n / 2$, if the quantity in (6.3) is finite then $\sigma$ is continuous and bounded with

$$
\sup _{\mathbb{R}^{n}}|\sigma| \lesssim\|\sigma\|_{l . u . H^{s}\left(\mathbb{R}^{n}\right), \eta} .
$$

Furthermore

$$
s>n / 2 \Longrightarrow\|\sigma\|_{l . u . H^{s}\left(\mathbb{R}^{n}\right), \eta} \asymp \sup _{\mathbb{R}^{n}}|\sigma|+\sup _{r>0} r^{s-n / 2}\left\|\sigma \eta\left(r^{-1} \cdot\right)\right\|_{H^{s}\left(\mathbb{R}^{n}\right)},
$$

having denoted $\dot{H}^{s}\left(\mathbb{R}^{n}\right)$ the homogeneous Sobolev space on $\mathbb{R}^{n}$. Hörmander's multipliers theorem is sharp in the range of $s$, even when restricted to radial multipliers. In fact the sharpness may be obtained by considering the imaginary power $\operatorname{Op}\left(|\xi|^{2 i \alpha}\right)=\Delta^{i \alpha}$ of the Laplace operator $\Delta=-\partial_{1}^{2}-\ldots-\partial_{n}^{2}$, see [24, p.1746] and [26, p.51-52].

The Hörmander condition with $s$ near enough $n / 2$ implies Mihlin's condition in (6.1) since we check easily

$$
s \in \mathbb{N} \Longrightarrow\|\sigma\|_{l . u . H^{s}\left(\mathbb{R}^{n}\right), \eta} \lesssim \max _{\alpha \in \mathbb{N}_{0}^{n},|\alpha| \leq s} \sup _{\xi \in \mathbb{R}^{n}}|\xi|^{\alpha}\left|\partial^{\alpha} \sigma(\xi)\right|
$$

In the next section, we will define a Hörmander condition thanks to the equivalence (6.5). The main issue is to define a relevant cut-off function $\eta$ that can be dilated. On $\mathbb{R}^{n}$, we can restrict ourselves to radial cut-offs, i.e. $\eta=\eta_{1}\left(|\cdot|^{2}\right)$, which can be viewed as the Fourier transform of the spectral multiplier $\eta_{1}(\Delta)$ of the Laplace operator on $\mathbb{R}^{n}$. An analogue cut-off function as spectral multiplier in $\mathcal{L}$ can be defined in our context; it can also be dilated.

6.2. The space $\mathcal{M}_{s}$. The previous section leads us to define the space $\mathcal{M}_{s}$ of symbol satisfying the following condition of Hörmander type:

Definition 6.4. Let $s>n / 2$ and $\eta \in \mathcal{D}(0, \infty)$. We denote by $\mathcal{M}_{s, \eta}$ the space of bounded symbols $\sigma \in L^{\infty}(\widehat{G}: \Sigma)$ such that the following quantity is finite:

$$
\sup _{r>0} r^{s-\frac{n}{2}}\left\|\sigma \eta\left(r^{-2} \widehat{\mathcal{L}}\right)\right\|_{\dot{H}^{s}(\widehat{G})} .
$$

We then set

$$
\|\sigma\|_{\mathcal{M}_{s}, \eta}:=\|\sigma\|_{L^{\infty}(\widehat{G}: \Sigma)}+\sup _{r>0} r^{s-\frac{n}{2}}\left\|\sigma \eta\left(r^{-2} \widehat{\mathcal{L}}\right)\right\|_{\dot{H}^{s}(\widehat{G})} .
$$

In dealing with these spaces we will often use the fact that in the supremum above we may assume $r$ large: 
Lemma 6.5. Let $s>n / 2$ and $\eta \in \mathcal{D}(0, \infty)$. For any $r_{0}>0$, there exists $C>0$ depending only on $r_{0}, s, \eta$ and the structure of the group $G$ such that we have for any $\sigma \in \Sigma$ :

$$
\|\sigma\|_{\mathcal{M}_{s}, \eta} \leq C\left(\|\sigma\|_{L^{\infty}(\widehat{G}: \Sigma)}+\sup _{r>r_{0}} r^{s-\frac{n}{2}}\left\|\sigma \eta\left(r^{-2} \widehat{\mathcal{L}}\right)\right\|_{H^{s}(\widehat{G})}\right),
$$

in the sense that if the right-hand side is finite then the left-hand side is finite and the inequality holds.

Proof. Let $\eta \in \mathcal{D}(0, \infty)$ with supp $\eta \subset\left(a_{0}, b_{0}\right) \subset(0, \infty)$. By Proposition 4.9 Part (2),

$$
\left\|\sigma \eta\left(r^{-2} \widehat{\mathcal{L}}\right)\right\|_{H^{s}(\widehat{G})} \lesssim\left\|\sigma \eta\left(r^{-2} \widehat{\mathcal{L}}\right)\right\|_{\dot{H}^{[s]}(\widehat{G})}=\sqrt{\sum_{|\alpha|=s}\left\|\Delta^{\alpha} \sigma \eta\left(r^{-2} \widehat{\mathcal{L}}\right)(\pi)\right\|_{L^{2}\left(\widehat{G}, \Sigma_{\varphi} \otimes \alpha\right.}^{2}} .
$$

By Lemma 3.8 Part (4), if $r \leq r_{0}$ the summation over $\pi \in \widehat{G}$ is in fact over a finite number of $\pi \in \widehat{G}$ since they will satisfy $\lambda_{\pi}<C+r_{0}^{2} b_{0}$. Using

$$
\left\|\Delta^{\alpha} \sigma \eta\left(r^{-2} \widehat{\mathcal{L}}\right)(\pi)\right\|_{L^{2}\left(\widehat{G}, \Sigma_{\varphi} \otimes \alpha\right)} \leq C_{\pi, \alpha}\|\sigma\|_{L^{\infty}(\widehat{G}: \Sigma)},
$$

we obtain

$$
r \leq r_{0} \Longrightarrow\left\|\sigma \eta\left(r^{-2} \widehat{\mathcal{L}}\right)\right\|_{\dot{H}^{[s]}(\widehat{G})} \leq C_{[s], G, C, a_{0}, r_{0}}^{\prime}\|\sigma\|_{L^{\infty}(\widehat{G}: \Sigma)} .
$$

The statement follows.

The definition of $\mathcal{M}_{s, \eta}$ is independent of any (non trivial) $\eta$ and $\|\sigma\|_{\mathcal{M}_{s}, \eta} \asymp\|\sigma\|_{\Sigma_{\zeta}, \zeta}$ for any two non-trivial $\eta, \zeta \in \mathcal{D}(0, \infty)$ since we have:

Lemma 6.6. Let $s>n / 2$ and let $\eta, \zeta \in \mathcal{D}(0, \infty)$. We assume $\eta \not \equiv 0$. Then we have

$$
\|\sigma\|_{\mathcal{M}_{s}, \zeta} \leq C\|\sigma\|_{\mathcal{M}_{s}, \eta}
$$

where $C=C_{s, G, \eta, \zeta}>0$ is a constant independent of $\sigma \in \Sigma$, in the sense that if the left-hand side is finite, then the right-hand side is finite and the inequality holds.

Proof. Let $\eta$ and $\zeta$ as in the statement. We may assume $\eta$ real valued (otherwise we consider separately its real and imaginary parts). Let $c_{o}>0$ such that $2^{c_{o}} I$ intersects $I$ where $I$ is an open interval inside the support of $\eta$. For $\lambda \in \mathbb{R}$ and $j \in \mathbb{Z}$, we set

$$
\eta_{j}(\lambda)=\eta\left(2^{-c_{o} j} \lambda\right) \quad \text { and } \quad \alpha(\lambda):=\sum_{j \in \mathbb{Z}} \eta_{j}^{2}(\lambda) .
$$

We check easily that $\alpha \equiv 0$ on $(-\infty, 0]$ and that on $(0, \infty), \alpha$ is a smooth positive function which does not vanish. Furthermore

$$
\forall \lambda \in \mathbb{R}, j \in \mathbb{Z} \quad \alpha\left(2^{j c_{o}} \lambda\right)=\alpha(\lambda), \quad \text { thus } \quad \forall \lambda>0 \quad \sum_{j \in \mathbb{Z}} \frac{\eta_{j}^{2}}{\alpha}(\lambda)=1 .
$$

Hence for any $\pi \in \widehat{G} \backslash\left\{1_{\widehat{G}}\right\}$, we have $\mathrm{I}_{\mathcal{H}_{\pi}}=\sum_{j \in \mathbb{Z}} \frac{\eta_{j}^{2}}{\alpha}\left(r^{-2} \pi(\mathcal{L})\right)$ in $\mathscr{L}\left(\mathcal{H}_{\pi}\right)$. Inserting the sum, we obtain

$$
\left\|\sigma \zeta\left(r^{-2} \widehat{\mathcal{L}}\right)\right\|_{\dot{H}^{s}(\widehat{G})} \leq \sum_{j \in \mathbb{Z}}\left\|\sigma\left(\frac{\eta_{j}^{2}}{\alpha} \zeta\right)\left(r^{-2} \widehat{\mathcal{L}}\right)\right\|_{\dot{H}^{s}(\widehat{G})} .
$$


In fact the sum is over $j$ such that $2^{j c_{o}}$ supp $\eta$ and supp $\zeta$ have a non-empty intersection, so this summation is finite and independent of $r$ and $\sigma$. Proposition 4.9 Part (1) and Lemma 4.14 yield

$$
\begin{aligned}
\left\|\sigma\left(\frac{\eta_{j}^{2}}{\alpha} \zeta\right)\left(r^{-2} \widehat{\mathcal{L}}\right)\right\|_{\dot{H}^{s}(\widehat{G})} \lesssim & \left\|\sigma \eta_{j}\left(r^{-2} \widehat{\mathcal{L}}\right)\right\|_{\dot{H}^{s}(\widehat{G})}\left\|\left(\frac{\eta_{j}}{\alpha} \zeta\right)\left(r^{-2} \mathcal{L}\right) \delta_{e_{G}}\right\|_{L^{1}(G)} \\
& +\left\|\sigma \eta_{j}\left(r^{-2} \widehat{\mathcal{L}}\right)\right\|_{L^{2}(\widehat{G}: \Sigma)}\left\|\left(\frac{\eta_{j}}{\alpha} \zeta\right)\left(r^{-2} \mathcal{L}\right) \delta_{e_{G}}\right\|_{L^{1}\left(|x|^{s} d x\right)} .
\end{aligned}
$$

By the Peter Weyl theorem,

$$
\left\|\sigma \eta_{j}\left(r^{-2} \widehat{\mathcal{L}}\right)\right\|_{L^{2}(\widehat{G}: \Sigma)} \leq\|\sigma\|_{L^{\infty}(\widehat{G}: \Sigma)}\left\|\eta_{j}\left(r^{-2} \widehat{\mathcal{L}}\right)\right\|_{L^{2}(\widehat{G}: \Sigma)} \lesssim\|\sigma\|_{L^{\infty}(\widehat{G}: \Sigma)}\left(2^{j} r^{2}\right)^{\frac{n}{4}}
$$

by Theorem 5.3 Part (3), which also provides estimates for the $L^{1}$-norms in $\left(\frac{\eta_{j}}{\alpha} \zeta\right)\left(r^{-2} \mathcal{L}\right) \delta_{e_{G}}$. We obtain

$$
\left\|\sigma \zeta\left(r^{-2} \widehat{\mathcal{L}}\right)\right\|_{\dot{H}^{s}(\widehat{G})} \lesssim\|\sigma\|_{\mathcal{M}_{s}, \eta} r^{-\left(s-\frac{n}{2}\right)} .
$$

This implies $\|\sigma\|_{\mathcal{M}_{s, \zeta}} \lesssim\|\sigma\|_{\mathcal{M}_{s}, \eta}$ and concludes the proof.

Hence we may write

$$
\mathcal{M}_{s}:=\mathcal{M}_{s, \eta} \text { for any non-trivial } \eta \in \mathcal{D}(0, \infty) .
$$

A first criteria of membership of $\mathcal{M}_{s}$ independent of a choice of a $\sigma \in \mathcal{D}(0, \infty)$ is given by the following property:

Lemma 6.7. For any $0<a_{0}<b_{0}, s>n / 2$ and any $\eta \in \mathcal{D}(0, \infty)$, there exists $C=$ $C_{s, a_{0}, b_{0}, \eta}>0$ such that we have for any $\sigma \in \Sigma$ :

$$
\|\sigma\|_{\mathcal{M}_{s}, \eta} \leq C\left(\|\sigma\|_{L^{\infty}(\widehat{G}: \Sigma)}+\sup _{r>0} r^{s-\frac{n}{2}}\left\|\sigma 1_{\left[r^{2} a_{0}, r^{2} b_{0}\right]}(\widehat{\mathcal{L}})\right\|_{H^{s}(\widehat{G})}\right) .
$$

Proof. First let us fix $0<a_{0}<b_{0}$ and consider $\eta \in \mathcal{D}(0, \infty)$ with $\eta \equiv 1$ on $\left[a_{0}, b_{0}\right]$. Then $\sigma \eta\left(r^{-2} \widehat{\mathcal{L}}\right)=\sigma_{r, a_{0}, b_{0}} \eta\left(r^{-2} \widehat{\mathcal{L}}\right)$ and we have by Lemma 4.14

$$
\begin{aligned}
& \left\|\sigma \eta\left(r^{-2} \widehat{\mathcal{L}}\right)\right\|_{\dot{H}^{s}(\widehat{G})}=\left\|\sigma_{r, a_{0}, b_{0}} \eta\left(r^{-2} \widehat{\mathcal{L}}\right)\right\|_{\dot{H}^{s}(\widehat{G})} \\
& \quad \lesssim\left\|\sigma_{r, a_{0}, b_{0}}\right\|_{\dot{H}^{s}(\widehat{G})}\left\|\eta\left(r^{-2} \mathcal{L}\right) \delta_{e_{G}}\right\|_{L^{1}(G)}+\left\|\sigma_{r, a_{0}, b_{0}}\right\|_{\dot{H}^{0}(\widehat{G})}\left\|\eta\left(r^{-2} \mathcal{L}\right) \delta_{e_{G}}\right\|_{L^{1}\left(|x|^{s} d x\right)} .
\end{aligned}
$$

Using Theorem 5.3 Part (3) to estimate the $L^{1}$-norms, and proceeding as in (6.7) for the $\dot{H}^{0}(\widehat{G})=L^{2}(\widehat{G}: \Sigma)$-norm, we obtain:

$$
\left\|\sigma \eta\left(r^{-2} \widehat{\mathcal{L}}\right)\right\|_{H^{s}(\widehat{G})} \lesssim\left\|\sigma_{r, a_{0}, b_{0}}\right\|_{H^{s}(\widehat{G})}+\|\sigma\|_{L^{\infty}(\widehat{G}: \Sigma)} r^{-s+\frac{n}{2}} .
$$

This together with Lemma 6.6 implies the statement.

A criteria for membership in $\mathcal{M}_{s}$ analogue to (6.6) is the following:

Lemma 6.8. Let $s \in \mathbb{N}$, and let $\eta \in \mathcal{D}(0, \infty)$. There exists a constant $C=C_{s, G, \eta}>0$ such that for any multiplier $\sigma$ we have for any $r>0$

$$
\|\sigma\|_{\mathcal{M}_{s}, \eta} \leq C \max _{\substack{\alpha \in \mathbb{N}_{0}^{f} \\|\alpha| \leq s}} \sup _{\pi \in \widehat{G}}\left(1+\lambda_{\pi}\right)^{\frac{|\alpha|}{2}}\left\|\Delta^{\alpha} \sigma(\pi)\right\|_{\mathscr{L}\left(\mathcal{H}_{\varphi \otimes \alpha} \otimes \mathcal{H}_{\pi}\right)},
$$

in the sense that if the left-hand side is finite then $\eta\left(r^{-2} \widehat{\mathcal{L}}\right) \sigma \in \dot{H}^{s}(\widehat{G})$ is finite for any $r>0$ and the inequality holds. 
Proof. Let $s \in \mathbb{N}$ with $s>n / 2$. Let $\eta \in \mathcal{D}(0, \infty)$. By Lemmata 6.5 and 6.6, we may assume supp $\eta=\left[\frac{1}{2}, 2\right]$.

The Leibniz formula (2.4) implies

$$
\begin{aligned}
& \left.\sum_{|\alpha|=s}\left\|\Delta^{\alpha}\left(\sigma \eta\left(r^{-2} \widehat{\mathcal{L}}\right)\right)(\pi)\right\|_{H S\left(\mathcal{H}_{\varphi} \otimes \alpha\right.} \otimes \mathcal{H}_{\pi}\right) \\
& \quad \sum_{s} \sum_{\left|\alpha_{1}\right|+\alpha_{2} \mid=s}\left\|\Delta^{\alpha_{1}} \sigma(\pi)\right\|_{\mathscr{L}\left(\mathcal{H}_{\varphi} \otimes \alpha_{1} \otimes \mathcal{H}_{\pi}\right)}\left\|\Delta^{\alpha_{2}} \sigma(\pi)\right\|_{H S\left(\mathcal{H}_{\varphi} \otimes \alpha_{2} \otimes \mathcal{H}_{\pi}\right)}
\end{aligned}
$$

By Lemma 3.8 Part (4), the supremum $C_{0}:=\sup \left|\lambda_{\rho}-\lambda_{\pi}\right|$ over $\pi, \rho \in \widehat{G}$ and $\alpha_{2} \in \mathbb{N}_{0}^{f}$, $\left|\alpha_{2}\right| \leq s$ such that $\rho \subset \varphi^{\otimes \alpha_{2}} \otimes \pi$ is finite. Hence we have

$$
\Delta^{\alpha_{2}} \eta\left(r^{-2} \widehat{\mathcal{L}}\right)(\pi)=0 \text { when } \lambda_{\pi} \notin\left[\frac{r^{2}}{2}-C_{0}, 2 r^{2}+C_{0}\right] .
$$

We fix $r_{0}>0$ such that $1<\frac{r^{2}}{2}-C_{0}<2 r^{2}+C_{0}$ for all $r>r_{0}$. Assuming that

$$
M_{s^{\prime}}:=\max _{\left|\alpha_{1}\right|=s^{\prime}} \sup _{\pi \in \widehat{G}}\left(1+\lambda_{\pi}\right)^{\frac{s^{\prime}}{2}}\left\|\Delta^{\alpha_{1}} \sigma(\pi)\right\|_{\mathscr{L}\left(\mathcal{H}_{\varphi} \otimes \alpha_{1} \otimes \mathcal{H}_{\pi}\right)},
$$

is finite for $s^{\prime}=0, \ldots, s$, we have for every $r>r_{0}$ :

$$
\sum_{|\alpha|=s}\left\|\Delta^{\alpha}\left(\sigma \eta\left(r^{-2} \widehat{\mathcal{L}}\right)\right)(\pi)\right\|_{H S\left(\mathcal{H}_{\varphi} \otimes \alpha \otimes \mathcal{H}_{\pi}\right)} \lesssim s \sum_{\left|\alpha_{2}\right|+s^{\prime}=s} M_{s^{\prime}} r^{s^{\prime}}\left\|\Delta^{\alpha_{2}} \sigma(\pi)\right\|_{H S\left(\mathcal{H}_{\varphi} \otimes \alpha_{2} \otimes \mathcal{H}_{\pi}\right)}
$$

and

$$
\begin{aligned}
& \left\|\sigma \eta\left(r^{-2} \widehat{\mathcal{L}}\right)\right\|_{\dot{H}^{s}(\widehat{G})}^{2}=\sum_{|\alpha|=s} \sum_{\pi \in \widehat{G}} d_{\pi}\left\|\Delta^{\alpha}\left(\sigma \eta\left(r^{-2} \widehat{\mathcal{L}}\right)\right)(\pi)\right\|_{H S\left(\mathcal{H}_{\varphi} \otimes \ldots \otimes \mathcal{H}_{\tau_{s}} \otimes \mathcal{H}_{\pi}\right)}^{2} \\
& \quad \max _{s^{\prime}=0, \ldots, s} M_{s^{\prime}}^{2} r^{2 s^{\prime}}\left\|\eta\left(r^{-2} \widehat{\mathcal{L}}\right)\right\|_{\dot{H}^{s-s^{\prime}}(\widehat{G})}^{2} .
\end{aligned}
$$

By Lemma 4.6 and Theorem 5.3 Part (3), we have

$$
\left\|\eta\left(r^{-2} \widehat{\mathcal{L}}\right)\right\|_{\dot{H}^{s-s^{\prime}}(\widehat{G})}=\left\|\eta\left(r^{-2} \mathcal{L}\right) \delta_{e_{G}}\right\|_{L^{2}\left(q_{1}^{s-s^{\prime}}\right)} \asymp\left\|\eta\left(r^{-2} \mathcal{L}\right) \delta_{e_{G}}\right\|_{L^{2}\left(|x|^{2\left(s-s^{\prime}\right)} d x\right)} \lesssim r^{s-s^{\prime}-\frac{n}{2}}
$$

We have obtained for any $r>r_{0}$

$$
\left\|\sigma \eta\left(r^{-2} \widehat{\mathcal{L}}\right)\right\|_{\dot{H}^{s}(\widehat{G})} \lesssim s \max _{s^{\prime}=0, \ldots, s} M_{s^{\prime}} r^{s^{\prime}} r^{s-s^{\prime}-\frac{n}{2}}=\max _{s^{\prime}=0, \ldots, s} M_{s^{\prime}} r^{s-\frac{n}{2}}
$$

By Lemma 6.5, this concludes the proof.

The spectral multiplier in the Laplace-Beltrami operator provides examples of multipliers in $\mathcal{M}_{s}$ :

Proposition 6.9. Let $G$ be a compact Lie group of dimension $n$. Let $s^{\prime}>s>\frac{n}{2}$. For every $f$ locally uniformly in $H^{s^{\prime}}(\mathbb{R})$, the spectral multiplier $f(\widehat{\mathcal{L}})$ is in $\mathcal{M}_{s}$. Moreover for every non-trivial $\eta, \eta_{1} \in \mathcal{D}(0, \infty)$ there exists a constant $C>0$ independent of $f$ such that

$$
\|f(\widehat{\mathcal{L}})\|_{\mathcal{M}_{s}, \eta} \leq C\|f\|_{l . u . H^{s^{\prime}}(\mathbb{R}), \eta_{1}} .
$$

The norm $\|\cdot\|_{l . u . H^{s^{\prime}}(\mathbb{R}), \eta}$ was defined via $(\underline{6.3})$ on $\mathbb{R}^{n}$. 
Proof. The properties of the functional calculus and (6.4) imply

$$
\|f(\widehat{\mathcal{L}})\|_{L^{\infty}(\widehat{G}: \Sigma)} \leq\|f\|_{L^{\infty}(\mathbb{R})} \lesssim\|f\|_{l \cdot u \cdot H^{s^{\prime}}(\mathbb{R}), \eta} .
$$

Let us apply Proposition 5.2 with a function $\eta_{1} \in \mathcal{D}(0, \infty)$ to the function $\lambda \mapsto f(\lambda) \eta\left(r^{-2} \lambda\right)$ :

$$
\left\|f(\widehat{\mathcal{L}}) \eta\left(r^{-2} \widehat{\mathcal{L}}\right)\right\|_{\dot{H}^{s}(\widehat{G})} \lesssim \sup _{r_{1}>0}\left\|f\left(r_{1} \cdot\right) \eta\left(r^{-2} r_{1} \cdot\right) \eta_{1}\right\|_{H^{s^{\prime}}(\mathbb{R})} \cdot
$$

Since the mapping $\psi \mapsto \psi \chi$ is continuous on $H^{s^{\prime}}(\mathbb{R})$ for any function $\chi \in \mathcal{D}(\mathbb{R})$, we obtain easily

$$
\sup _{r>0}\left\|f(\widehat{\mathcal{L}}) \eta\left(r^{-2} \widehat{\mathcal{L}}\right)\right\|_{\dot{H}^{s}(\widehat{G})} \lesssim \sup _{r, r_{1}>0}\left\|f\left(r_{1} \cdot\right) \eta\left(r^{-2} r_{1} \cdot\right) \eta_{1}\right\|_{H^{s^{\prime}}(\mathbb{R})} \lesssim \sup _{r>0}\left\|f\left(r_{1} \cdot\right) \eta_{1}\right\|_{H^{s^{\prime}}(\mathbb{R})} \cdot
$$

Therefore, we have:

$$
\|f(\widehat{\mathcal{L}})\|_{\mathcal{M}_{s}, \eta} \leq C\|f\|_{l . u . H^{s^{\prime}}(\mathbb{R}), \eta_{1}}
$$

We conclude with the equivalence of two norms $\|\cdot\|_{l . u . H^{s^{\prime}}(\mathbb{R}), \eta}$ for two non trivial functions $\eta \in \mathcal{D}(0, \infty)$.

6.3. Multiplier theorems on $G$. The main result of the paper are the analogues of both Mihlin and Hörmander-type conditions for the Fourier multiplier theorem.

We start with the analogue of the Hörmander-type condition. This will use the space $\mathcal{M}_{s}$ defined in Section 6.2.

Theorem 6.10 (Hormander-type multiplier theorem). Let $G$ be a compact Lie group of dimension $n$. If the multiplier $\sigma=\{\sigma(\pi), \pi \in \widehat{G}\}$ is in $\mathcal{M}_{s}$ for some $s>n / 2$ then the Fourier multiplier operator $\mathrm{Op}(\sigma)$ is bounded on $L^{p}(G)$ for any $1<p<\infty$, and $L^{1}-L^{1, \infty}$ for $p=1$. Furthermore, for $p \in(1, \infty)$,

$$
\|\mathrm{Op}(\sigma)\|_{\mathscr{L}\left(L^{p}(G)\right)} \leq C_{\eta, p, G}\|\sigma\|_{\mathcal{M}_{s, \eta}}
$$

for some non-zero $\eta \in \mathcal{D}(0, \infty)$, where the constant $C_{\eta, p, G}$ depends on $\eta, p$ and the structure of $G$ but not on $\sigma$. We have a similar bound for $p=1$.

As in the case of Fourier multipliers on $\mathbb{R}^{n}$, Theorem 6.10 is sharp in $s$ for Fourier multipliers even when restricted to spectral multipliers in $\mathcal{L}$. Indeed by Theorem 6.10 and Proposition 6.9 we have

$$
\forall s^{\prime}>n / 2, \forall \alpha \in \mathbb{R} \quad\left\|\mathcal{L}^{i \alpha}\right\|_{\mathscr{L}\left(L^{1}(G), L^{1, \infty}(G)\right)} \lesssim_{s^{\prime}, G}\left\|\lambda^{i \alpha}\right\|_{l . u \cdot H^{s^{\prime}}(\mathbb{R}), \eta} \lesssim_{s^{\prime}, G}(1+|\alpha|)^{s^{\prime}}
$$

whereas the arguments of Sikora and Wright [24, Theorem 1] show that

$$
\forall \alpha \in \mathbb{R} \quad\left\|\mathcal{L}^{i \alpha}\right\|_{\mathscr{L}\left(L^{1}(G), L^{1, \infty}(G)\right)} \gtrsim_{G}(1+|\alpha|)^{n / 2} .
$$

Note that Theorem 6.10 and Proposition 6.9 yield the Hörmander theorem for spectral multipliers of a Laplace-beltrami $\mathcal{L}$ on $G$, but this result is a particular case of the celebrated result of Seeger and Sogge valid for a much larger class of operators on any compact manifold [22].

We now give the Mihlin-type Fourier multiplier theorem which as in the case of $\mathbb{R}^{n}$ can be obtained from the Hörmander version: indeed Theorem 6.11 follows readily from Theorem 6.10 together with Lemma 6.8: 
Theorem 6.11 (Mihlin-type multiplier theorem). Let $G$ be a compact Lie group of dimension n. Let $\sigma \in L^{\infty}(\widehat{G}, \Sigma)$. If there exists a constant $M \geq 0$ such that

$$
\forall \pi \in \widehat{G}, \quad \forall \alpha \in \mathbb{N}_{0}^{f},|\alpha| \leq[n / 2]+1 \quad\left\|\Delta^{\alpha} \sigma(\pi)\right\|_{\mathscr{L}\left(\mathcal{H}_{\varphi \otimes} \otimes \mathcal{H}_{\pi}\right)} \leq M\left(1+\lambda_{\pi}\right)^{-\frac{|\alpha|}{2}},
$$

then the Fourier multiplier operator $\operatorname{Op}(\sigma)$ is bounded on $L^{p}(G)$ for any $1<p<\infty$, and $L^{1}-L^{1, \infty}$ for $p=1$. Furthermore, for $p \in(1, \infty)$,

$$
\|\mathrm{Op}(\sigma)\|_{\mathscr{L}\left(L^{p}(G)\right)} \leq C_{p, G}\left(\|\sigma\|_{L^{\infty}(\widehat{G}: \Sigma)}+\max _{s^{\prime}=1, \ldots, s} M_{s^{\prime}}\right) .
$$

where the constant $C_{p, G}$ depends on $p$ and the structure of $G$ but not on $\sigma$. We have a similar bound for $p=1$.

Note that Theorem 6.11] is sharper than the main result in [21] of Ruzhansky and Wirth who obtain a similar result but with $s^{\prime}$ being an even integer strictly greater than $n / 2$. Furthermore the results of [21] applied to the case of the torus $\mathbb{T}$ do not recover the original 1939 result of Marcinkiewicz (Theorem 6.1), whereas it will be the case for the Marcinkiewicz type theorem proved in Section 6.5, as a consequence of (the proof of) Theorem 6.10.

6.4. Proof of Theorem 6.10. Again as in the case of $\mathbb{R}^{n}$, to show the Hörmander version of our Fourier multiplier theorem (Theorem 6.10), it suffices to prove the case ' $r=2^{j / 2}$, that is:

Proposition 6.12. Let $G$ be a compact Lie group of dimension n. Let $\sigma=\{\sigma(\pi), \pi \in \widehat{G}\} \in$ $L^{\infty}(\widehat{G}: \Sigma)$ be a bounded multiplier. If there exists a constant $C_{0}>0$ satisfying

$$
\forall j \in \mathbb{Z} \quad\left\|\sigma \eta\left(2^{-j} \widehat{\mathcal{L}}\right)\right\|_{\dot{H}^{s}(\widehat{G})} \leq C_{0} 2^{\frac{j}{2}\left(\frac{n}{2}-s\right)},
$$

for some $s>n / 2$ and some non-zero $\eta \in \mathcal{D}(0, \infty)$, then the Fourier multiplier operator $\operatorname{Op}(\sigma)$ is bounded on $L^{p}(G)$ for any $1<p<\infty$, and $L^{1}-L^{1, \infty}$ for $p=1$. Furthermore, for $p \in(1, \infty)$,

$$
\|\mathrm{Op}(\sigma)\|_{\mathscr{L}\left(L^{p}(G)\right)} \leq C_{p, G}\left(\|\sigma\|_{L^{\infty}(\widehat{G}: \Sigma)}+C_{0}\right) .
$$

where the constant $C_{p, G}$ depends on $p$ and the structure of $G$ but not on $\sigma$. We have a similar bound for $p=1$.

In this section, we prove Proposition 6.12, thereby proving the main result of this paper, i.e. Theorem 6.10. The method is classical, and we allow ourselves to sketch the ideas rather than dwelling on technical details.

We choose the function $\eta \in \mathcal{D}(0, \infty)$ yielding a dyadic decomposition:

$$
\operatorname{supp} \eta \subset(1,2) \quad \text { and } \quad \forall \lambda>0 \quad \sum_{j \in \mathbb{Z}} \eta\left(2^{-j} \lambda\right)=1,
$$

and we set $\eta_{j}(\lambda):=\eta\left(2^{-j} \lambda\right)$. Note that since the spectrum of $\mathcal{L}$ is a discrete subset of $[0, \infty)$ with no accumulation point, there exists $j_{0}$ depending on $\eta$ and $G$, such that $\eta_{j}(\widehat{\mathcal{L}})=0$ for all $j<j_{0}$.

Note that if $\sigma=\delta_{\pi=1_{\widehat{G}}}$ in the sense that $\sigma(\pi)=0$ unless $\pi=1_{\widehat{G}}$ and $\sigma\left(1_{\widehat{G}}\right)=1$, then the inversion formula yields $\operatorname{Op}\left(\delta_{\pi=1_{\widehat{G}}}\right) \phi \equiv \int_{G} \phi$ for any $\phi \in \mathcal{D}(G)$ so that $\left\|\operatorname{Op}\left(\delta_{\pi=1_{\widehat{G}}}\right)\right\|_{\mathscr{L}\left(L^{p}(G)\right)} \leq$ 
1 for any $p \in[1, \infty)$. Therefore it suffices to prove Proposition 6.12 for $\sigma \in L^{\infty}(\widehat{G}: \Sigma)$ satisfying $\sigma\left(1_{\widehat{G}}\right)=0$.

Let $\sigma \in L^{\infty}(\widehat{G}: \Sigma)$ such that $\sigma\left(1_{\widehat{G}}\right)=0$. We assume that there exist $s>n / 2$ and $C_{0}>0$ such that

$$
\forall j \in \mathbb{Z} \quad\left\|\sigma_{j}\right\|_{\dot{H}^{s}(\widehat{G})} \leq C_{0} 2^{-\frac{j}{2}\left(s-\frac{n}{2}\right)},
$$

where $\sigma_{j}:=\sigma \eta_{j}(\widehat{\mathcal{L}})$. The sum $\sum_{j} \mathrm{Op}\left(\sigma_{j}\right)$ converges to $\mathrm{Op}(\sigma)$ in the strong operator topology of $\mathscr{L}\left(L^{2}(G)\right)$.

Let $\kappa, \kappa_{j} \in \mathcal{D}^{\prime}(G)$ be such that $\sigma=\widehat{\kappa}$ and $\sigma_{j}=\widehat{\kappa}_{j}$. The sum $\sum_{j} \kappa_{j}$ converges to $\kappa$ in $\mathcal{D}^{\prime}(G)$. Since $\kappa_{j} \in L_{\text {fin }}^{2}(\widehat{G}: \Sigma)$ is smooth, by hypothesis and Proposition 4.9 Part (1), $\kappa_{j} \in L^{2}\left(|x|^{2 s} d x\right)$ with

$$
\left\|\kappa_{j}\right\|_{L^{2}\left(|x|^{2 s} d x\right)} \asymp\left\|\sigma_{j}\right\|_{\dot{H}^{s}(\widehat{G})} \leq C_{0} 2^{\frac{j}{2}\left(\frac{n}{2}-s\right)} .
$$

So $\sum_{j}\left\|\kappa_{j}\right\|_{L^{2}\left(|x|^{2 s} d x\right)}<\infty$ and $\kappa$ coincides with a locally integrable function on $G \backslash\left\{e_{G}\right\}$ in $L^{2}\left(|x|^{2 s} d x\right)$, see Proposition 4.9 Part (1). The sum $\sum_{j} \kappa_{j}$ converges to $\kappa \in L^{2}\left(|x|^{2 s} d x\right)$ in $L^{2}\left(|x|^{2 s} d x\right)$.

By the fundamental theorem of singular integral [4, Ch. III], it suffices to show that

$$
\int_{\left|y^{-1} y^{\prime}\right| \lesssim\left|y^{-1} x\right|}\left|\kappa\left(y^{-1} x\right)-\kappa\left(y^{-1} x\right)\right| d x
$$

is finite independently of $y \neq y^{\prime} \in G$, and similarly for $\kappa^{*}: x \mapsto \bar{\kappa}\left(z^{-1}\right)$. We will give the proof for $\kappa$ only, the proof for $\kappa^{*}$ being similar.

It suffices to show that the sum

$$
\sum_{j=j_{0}}^{\infty} I_{j}(h), \quad \text { where } \quad I_{j}(h):=\int_{|h| \lesssim|z|}\left|\kappa_{j}(z)-\kappa_{j}(h z)\right| d z,
$$

is bounded independently of $h \in G \backslash\left\{e_{G}\right\}$.

We see that

$$
I_{j}(h) \leq 2 \int_{|h| \lesssim|z|}\left|\kappa_{j}(z)\right| d z \leq 2\left\|\kappa_{j}\right\|_{L^{2}\left(|x|^{2 s} d x\right)}\left\|1_{|h| \lesssim|z|}\right\|_{L^{2}\left(|z|^{-2 s} d z\right)},
$$

by the Cauchy-Schwartz inequality. We compute easily

$$
\left\|1_{|h| \lesssim|z|}\right\|_{L^{2}\left(|z|^{-2 s} d z\right)}^{2} \asymp \int_{r \sim|h|}^{1} r^{-2 s+n-1} d r \asymp 1+|h|^{-2 s+n} .
$$

Using (6.8), we obtain:

$$
I_{j}(h) \lesssim C_{0} 2^{-\frac{j}{2}\left(s-\frac{n}{2}\right)}\left(1+|h|^{-s+\frac{n}{2}}\right), \quad \text { and } \sum_{j:|h|^{-1} \sum^{j / 2}} \int_{|h| \lesssim|z|}\left|\kappa_{j}(z)-\kappa_{j}(h z)\right| d z \lesssim C_{0},
$$

independently of $h$.

Hence the main problem is the sum for $j \geq j_{0}$ such that $2^{j / 2} \lesssim|h|^{-1}$. We may also assume that $|h|$ is small, and thus that $h$ is in a fixed chart of $e_{G}$ provided by the exponential mapping. We have

$$
I_{j}(h) \leq \sum_{\ell=0}^{m} I_{j, \ell}(h), \quad \text { where } \quad I_{j, \ell}(h):=\int_{|z| \asymp 2^{-\ell}}\left|\kappa_{j}(z)-\kappa_{j}(h z)\right| d z,
$$


and $m \in \mathbb{N}_{0}$ is such that $|h| \asymp 2^{-m}$.

The estimate for the Taylor reminder of order 1 then yields:

$$
I_{j, \ell}(h) \lesssim|h| \int_{|z| \asymp 2^{-\ell}}\left|\nabla \kappa_{j}(z)\right| d z \quad \text { if } \ell \lesssim m .
$$

The Cauchy-Schwartz inequality implies

$$
\int_{|z| \asymp 2^{-\ell}}\left|\nabla \kappa_{j}(z)\right| d z \leq\left\|\nabla \kappa_{j}\right\|_{L^{2}(G)}\left\|1_{|z| \asymp 2^{-\ell}}\right\|_{L^{2}(G)}
$$

We compute $\left\|1_{|z| \asymp 2^{-\ell}}\right\|_{L^{2}(G)} \asymp 2^{-\ell n / 2}$. The functional calculus of $\mathcal{L}$ yields $\left\|\nabla \kappa_{j}\right\|_{L^{2}(G)} \asymp$ $2^{j / 2}\left\|\kappa_{j}\right\|_{L^{2}(G)}$ and we have

$$
\left\|\kappa_{j}\right\|_{L^{2}(G)}=\left\|\sigma_{j}\right\|_{L^{2}(\widehat{G}: \Sigma)} \leq\|\sigma\|_{L^{\infty}(\widehat{G}: \Sigma)}\left\|\eta_{j}(\widehat{\mathcal{L}})\right\|_{\dot{H}^{0}(\widehat{G})} \lesssim\|\sigma\|_{L^{\infty}(\widehat{G}: \Sigma)} 2^{j \frac{n}{4}}
$$

by Proposition 5.1. So we have obtained:

$$
I_{j, \ell}(h) \lesssim|h| 2^{\frac{j}{2}}\|\sigma\|_{L^{\infty}(\widehat{G}: \Sigma)} 2^{j \frac{n}{4}} 2^{-\ell \frac{n}{2}}
$$

and the sum $\sum I_{j, \ell}(h)$ over $j, \ell \in \mathbb{Z}$ satisfying $0 \leq \ell \leq m$ and $j_{0} \leq j \leq 2 \ell$ is finite independently of $|h|$.

To sum over $\ell<j / 2$, we go back to

$$
\int_{|z| \asymp 2^{-\ell}}\left|\nabla \kappa_{j}(z)\right| d z \leq\left\||\cdot|^{s}\left|\nabla \kappa_{j}\right|\right\|_{L^{2}(G)}\left\||\cdot|^{-s} 1_{|z| \asymp 2^{-\ell}}\right\|_{L^{2}(G)} .
$$

We compute $\left\||\cdot|^{-s} 1_{|z| \asymp 2^{-\ell}}\right\|_{L^{2}(G)} \asymp 2^{\ell(s-n / 2)}$. We fix $\tilde{\eta} \in \mathcal{D}(0, \infty)$ such that $\tilde{\eta} \equiv 1$ on supp $\eta$. We set $\tilde{\eta}_{j}(\lambda)=\tilde{\eta}\left(2^{-j} \lambda\right)$. We have $\kappa_{j}=\tilde{\eta}_{j}(\mathcal{L}) \kappa_{j}=\kappa_{j} * \tilde{\eta}_{j}(\mathcal{L}) \delta_{e_{G}}$ and Lemma 4.14 yields:

$$
\left.\left\||\cdot|^{s}\left|\nabla \kappa_{j}\right|\right\|_{L^{2}(G)} \lesssim\left\||\cdot|^{s}\left|\kappa_{j}\right|\right\|_{L^{2}(G)}||\left|\nabla \tilde{\eta}_{j}(\mathcal{L}) \delta_{e_{G}}\right|\left\|_{L^{1}(G)}+\right\| \kappa_{j} \|_{L^{2}(G)}|||\cdot|^{s}\left|\nabla \tilde{\eta}_{j}(\mathcal{L}) \delta_{e_{G}}\right|\right) \|_{L^{1}(G)} .
$$

Using (6.8) and (6.9) for the $L^{2}$-norm in $\kappa_{j}$, and Theorem 5.3 Part (3) for the $L^{1}$-norm with $\eta$ yields:

$\left\||\cdot|^{s}\left|\nabla \kappa_{j}\right|\right\|_{L^{2}(G)} \lesssim C_{0} 2^{\frac{j}{2}\left(\frac{n}{2}-s\right)}\left(2^{-j}\right)^{-\frac{1}{2}}+\|\sigma\|_{L^{\infty}(\widehat{G}: \Sigma)} 2^{j n / 4}\left(2^{-j}\right)^{\frac{s-1}{2}}=\left(C_{0}+\|\sigma\|_{L^{\infty}(\widehat{G}: \Sigma)}\right) 2^{\frac{j}{2}\left(\frac{n}{2}-s+1\right)}$.

Hence we have obtained

$$
I_{j, \ell}(h) \lesssim|h|\left(C_{0}+\|\sigma\|_{L^{\infty}(\widehat{G}: \Sigma)}\right) 2^{\frac{j}{2}\left(\frac{n}{2}-s+1\right)} 2^{\ell\left(s-\frac{n}{2}\right)},
$$

and the sum $\sum I_{j, \ell}(h)$ over $j, \ell \in \mathbb{Z}$ satisfying $0 \leq \ell \leq j / 2$ and $j_{0} \leq j \leq 2 m$ is finite independently of $h$.

This concludes the proof of Proposition 6.12, and of Theorem 6.10.

6.5. A Marcinkiewicz type condition. Our main result Theorem 6.10 , that is, the multiplier theorem of Hörmander type, is in term of the space $\mathcal{M}_{s}$ and can be viewed as a decay of $L^{2}$-weighted norms of the kernel localised in frequency. As a corollary, we obtained a Mihlin version in terms of decay of derivatives of the symbol, see Theorem 6.11, We now present a Marcinkiewicz-type theorem in the sense that the condition is expressed with the partial sum of derivatives being finite.

We will need the following notation: if $s_{0} \in \mathbb{N}$ and $\sigma \in \Sigma$, we set

$$
\|\sigma\|_{\dot{L}_{s_{0}}^{1}(\widehat{G}: \Sigma)}:=\sum_{|\alpha|=s_{0}} \sum_{\pi \in \widehat{G}} d_{\pi} \operatorname{Tr}_{\mathcal{H}_{\varphi} \otimes \alpha \otimes \mathcal{H}_{\pi}}\left|\Delta^{\alpha} \sigma(\pi)\right| .
$$


This quantity may be finite or infinite.

Remark 6.13. Because of the equivalence of matrix norms, we can replace the trace above with any norm of the finite dimensional space of endomorphism in $\mathcal{H}_{\varphi \otimes \alpha}$ valued in the matrix space over $\mathcal{H}_{\pi}$ equipped with the Schatten norm

$$
\|M\|_{S_{1}\left(\mathcal{H}_{\pi}\right)}:=\operatorname{Tr}|M|=\operatorname{Tr}\left(M^{*} M\right)^{\frac{1}{2}} .
$$

The constants involved in this equivalence depend on the spaces $\mathcal{H}_{\varphi}, \varphi \in \operatorname{Fund}(G)$, so on the structure of $G$, and not on $\pi \in \widehat{G}$. So for instance we have:

$$
\begin{aligned}
\|\sigma\|_{\dot{L}_{s_{0}}^{1}(\widehat{G}: \Sigma)} & \asymp \sum_{|\alpha|=s_{0}} \sum_{\pi \in \widehat{G}} d_{\pi}\left\|\Delta^{\alpha} \sigma(\pi)\right\|_{\mathscr{L}\left(\mathcal{H}_{\varphi \otimes \alpha} \otimes, S_{1}\left(\mathcal{H}_{\pi}\right)\right)} \\
& \asymp \sum_{|\alpha|=s_{0}} \sum_{\pi \in \widehat{G}} d_{\pi} \sum_{i_{1}, j_{1}, \ldots, i_{s_{0}}, j_{s_{0}}}\left\|\left[\Delta^{\alpha} \sigma(\pi)\right]_{i_{1}, j_{1}, \ldots, i_{s_{0}}, j_{s_{0}}}\right\|_{S_{1}\left(\mathcal{H}_{\pi}\right)} .
\end{aligned}
$$

This quantity enables us to obtain the following $L^{\infty}$-weighted estimates:

Lemma 6.14. For any $s_{0} \in \mathbb{N}$, there exists a constant $C=C_{G, s_{0}}>0$ such that we have for all $\phi \in \mathcal{D}(G)$ :

$$
\max _{x \in G}|x|^{s_{0}}|\phi(x)| \leq C\|\widehat{\phi}\|_{\dot{L}_{s_{0}}^{1}(\widehat{G}: \Sigma)} .
$$

Proof of Lemma 6.14. Let $\phi \in \mathcal{D}(G)$. The Fourier inversion formula and the definition of the difference operators (see Section 2.3) imply

$$
[\varphi(x)-\mathrm{I}]_{i, j} \phi(x)=\sum_{\pi \in \widehat{G}} d_{\pi} \operatorname{Tr}\left[\Delta_{\varphi} \widehat{\phi}(\pi)\right]_{i, j}, \quad \text { so } \quad\left|[\varphi(x)-\mathrm{I}]_{i, j} \phi(x)\right| \leq \sum_{\pi \in \widehat{G}} d_{\pi} \operatorname{Tr}\left|\left[\Delta_{\varphi} \widehat{\phi}(\pi)\right]_{i, j}\right| .
$$

By Proposition 4.4 Part (1), we have

$$
\max _{x \in G}|x||\phi(x)| \asymp \max _{G} \sqrt{q}_{1}|\phi| \asymp \sum_{\varphi \in \operatorname{Fund}(G)} \sum_{i, j} \max _{x \in G}\left|[\varphi(x)-\mathrm{I}]_{i, j} \phi(x)\right|,
$$

and the computation above then yields:

$$
\max _{x \in G}|x||\phi(x)| \lesssim \sum_{\varphi \in \operatorname{Fund}(G)} \sum_{\pi \in \widehat{G}} d_{\pi} \sum_{i, j} \operatorname{Tr}\left|\left[\Delta_{\varphi} \widehat{\phi}(\pi)\right]_{i, j}\right|
$$

By Remark 6.13, this implies the result for $s_{0}=1$. The proof for $s_{0}>1$ is similar and is left to the reader.

The $L^{\infty}$-weighted estimates in Lemma 6.14 easily yields some $L^{2}$-weighted estimates of localised kernels, and we obtain:

Corollary 6.15. (1) For any $\eta \in \mathcal{D}(0, \infty), s>0$ and any $s_{0} \in \mathbb{N}$, there exists a constant $C=C_{s, G, s, s_{0}, \eta}>0$ such that for any $\sigma \in \Sigma$ and $r \geq 1$, we have

$$
\left\|\sigma \eta\left(r^{-2} \widehat{\mathcal{L}}\right)\right\|_{\dot{H}^{s}(\widehat{G})} \leq C\left(\left\|\sigma \eta\left(r^{-2} \widehat{\mathcal{L}}\right)\right\|_{\dot{L}_{s_{0}}^{1}(\widehat{G}: \Sigma)} r^{-s+s_{0}-\frac{n}{2}}+\|\sigma\|_{L^{\infty}(\widehat{G}: \Sigma)} r^{\frac{n}{2}-s}\right),
$$

in the sense that if $\sigma \|_{L^{\infty}(\widehat{G}: \Sigma)}$ and $\|\sigma\|_{\dot{L}_{s_{0}}^{1}(\widehat{G}: \Sigma)}$ are finite, then the inequality holds. 
(2) If $\sigma \in \Sigma$ is such that $\sigma \|_{L^{\infty}(\widehat{G}: \Sigma)}$ and $\|\sigma\|_{{\dot{s_{0}}}_{s_{0}}(\widehat{G}: \Sigma)}$ are finite with $s_{0} \in \mathbb{N}, s_{0} \leq n$, then $\sigma \in \Sigma_{s}$ for any $s>\frac{n}{2}$. Moreover we have

$$
\|\sigma\|_{\mathcal{M}_{s, \eta}} \leq C\left(\|\sigma\|_{L^{\infty}(\widehat{G}: \Sigma)}+\sup _{r \geq 1}\left\|\sigma \eta\left(r^{-2} \widehat{\mathcal{L}}\right)\right\|_{\dot{L}_{s_{0}}^{1}(\widehat{G}: \Sigma)}\right),
$$

where $\eta \in \mathcal{D}(0, \infty)$ is a fixed non-trivial function, and $C=C_{s, G, s, s_{0}, \eta}>0$ is a constant, both independent of $\sigma$.

Proof. Let $\sigma \in \Sigma$ and $\eta \in \mathcal{D}(0, \infty)$. We set $\kappa_{r}:=\mathcal{F}_{G}^{-1} \sigma \eta\left(r^{-2} \widehat{\mathcal{L}}\right) \in \mathcal{D}(G)$ for any $r>0$. By Proposition 4.9 Part (1),

$$
\left\|\sigma \eta\left(r^{-2} \widehat{\mathcal{L}}\right)\right\|_{H^{s}(\widehat{G})} \asymp\left\|\kappa_{r}\right\|_{L^{2}\left(|x|^{2 s} d x\right)} \leq\left\|\kappa_{r} 1_{|x| \leq r^{-1}}\right\|_{L^{2}\left(|x|^{2 s} d x\right)}+\left\|\kappa_{r} 1_{|x|>r^{-1}}\right\|_{L^{2}\left(|x|^{2 s} d x\right)} .
$$

By Lemma 6.14, we have:

$$
\left\||\cdot|{ }^{s_{0}} \kappa_{r}\right\|_{\infty} \lesssim\left\|\sigma \eta\left(r^{-2} \widehat{\mathcal{L}}\right)\right\|_{\dot{L}_{s_{0}}^{1}(\widehat{G}: \Sigma)},
$$

so for any $r \geq 1$

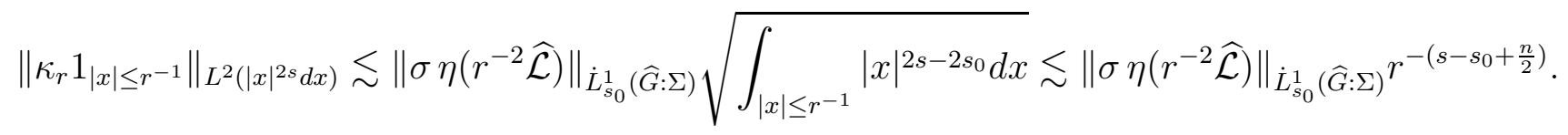

For the second $L^{2}$-norm, we have

$$
\left\|\kappa_{r} 1_{|x|>r^{-1}}\right\|_{L^{2}\left(|x|^{2 s} d x\right)} \leq r^{-s}\left\|\kappa_{r}\right\|_{L^{2}(G)},
$$

and by functional calculus and Theorem 5.3 Part (3),

$$
\left\|\kappa_{r}\right\|_{L^{2}(G)} \leq\|\sigma\|_{L^{\infty}(\widehat{G}: \Sigma)}\left\|\eta\left(r^{-2} \mathcal{L}\right) \delta_{e_{G}}\right\|_{L^{2}(G)} \lesssim\|\sigma\|_{L^{\infty}(\widehat{G}: \Sigma)} r^{\frac{n}{2}}
$$

Collecting the various estimates proves Part (1).

Part (2) follows easily from Part (1) and the properties of $\mathcal{M}_{s}$, see Section 6.2.

Corollary 6.15 Part (2) gives a sufficient condition for the membership of the symbol in $\mathcal{M}_{s}, s>n / 2$, therefore a sufficient condition for $L^{p}$-boundedness of the corresponding operator using Hörmander-type hypotheses in Theorem 6.10.

Here we choose to combine Proposition 6.12 with Corollary 6.15 Part (1) to obtain a sufficient condition 'with $r=2^{j / 2}$, which we view as a Marcinkiewicz-type property:

Theorem 6.16 (Marcinkiewicz-type multiplier theorem). Let $G$ be a compact Lie group of dimension $n$. If the symbol $\sigma=\{\sigma(\pi), \pi \in \widehat{G}\}$ is such that $\sigma \in L^{\infty}(\widehat{G}: \Sigma)$ and

$$
\exists s_{0} \in \mathbb{N}, s_{0} \leq n \quad \exists C_{0}>0 \quad \forall j \geq 0 \quad\left\|\sigma 1_{\left[2^{j}, 2^{j+1}\right]}(\widehat{\mathcal{L}})\right\|_{\dot{L}_{s_{0}}^{1}(\widehat{G}: \Sigma)} \leq C_{0} 2^{\frac{j}{2}\left(n-s_{0}\right)}
$$

then the Fourier multiplier operator $\operatorname{Op}(\sigma)$ is bounded on $L^{p}(G)$ for any $1<p<\infty$, and $L^{1}-L^{1, \infty}$ for $p=1$. Furthermore, for $p \in(1, \infty)$,

$$
\|\mathrm{Op}(\sigma)\|_{\mathscr{L}\left(L^{p}(G)\right)} \leq C_{s_{0}, p, G}\left(C_{0}+\|\sigma\|_{L^{\infty}(\widehat{G}: \Sigma)}\right)
$$

where the constant $C_{s_{0}, p, G}$ depends on $s_{0}, p$ and the structure of $G$ but not on $\sigma$. We have a similar bound for $p=1$. 
Proof. We fix $s>n / 2, \eta \in \mathcal{D}(0, \infty)$ and $j_{0} \in \mathbb{Z}$ as in the proof of Proposition 6.12. In particular, $\eta\left(2^{-j} \widehat{\mathcal{L}}\right)=0$ for $j<j_{0}$. Proceeding as in the proof of Lemma 6.7, we have for any $j \in \mathbb{Z}$ :

$$
\left\|\sigma \eta\left(2^{-j} \widehat{\mathcal{L}}\right)\right\|_{\dot{H}^{s}(\widehat{G})} \lesssim\left\|\sigma 1_{\left[2^{j}, 2^{j+1}\right]}(\widehat{\mathcal{L}})\right\|_{\dot{H}^{s}(\widehat{G})}+2^{-\frac{j}{2}\left(s-\frac{n}{2}\right)}\|\sigma\|_{L^{\infty}(\widehat{G}: \Sigma)},
$$

whereas proceeding as in the proof of Lemma 6.5, we have for any $j \in \mathbb{Z}, j<0$,

$$
\left\|\sigma \eta\left(2^{-j} \widehat{\mathcal{L}}\right)\right\|_{\dot{H}^{s}(\widehat{G})} \lesssim\|\sigma\|_{L^{\infty}(\widehat{G}: \Sigma)} .
$$

By Corollary 6.15 Part (1), we have for any $j \in \mathbb{N}_{0}$

$$
\left\|\sigma 1_{\left[2^{j}, 2^{j+1}\right]}(\widehat{\mathcal{L}})\right\|_{\dot{H}^{s}(\widehat{G})} \lesssim\left\|\sigma 1_{\left[2^{j}, 2^{j+1}\right]}(\widehat{\mathcal{L}})\right\|_{\dot{L}_{s_{0}}^{1}(\widehat{G}: \Sigma)^{2}} 2^{\frac{j}{2}\left(-s+s_{0}-\frac{n}{2}\right)}+\|\sigma\|_{L^{\infty}(\widehat{G}: \Sigma)^{2}} 2^{\frac{j}{2}\left(\frac{n}{2}-s\right)} .
$$

Combining all the estimates above together with the hypothesis of the statement implies that $\sigma$ satisfies the hypotheses of Proposition 6.12 and the conclusion follows.

Applying Theorem 6.16 to the one-dimensional torus $G=\mathbb{T}$ with $s_{0}=n=1$, we recover the historical 1939 theorem stated in Theorem 6.1.

\section{REFERENCES}

[1] Alexopoulos, G., Spectral multipliers on Lie groups of polynomial growth, Proc. Amer. Math. Soc. 120 (1994), No 3, pp 973-979.

[2] Beggs E.J. and Majid S., Quantum Riemannian Geometry, to appear.

[3] Clerc, J.-L., Sommes de Riesz et multiplicateurs sur un groupe de Lie compact, Ann. Inst. Fourier (Grenoble) 24 (1974), No 1, pp 149-172.

[4] Coifman, R. and Weiss, G., Analyse harmonique non-commutative sur certains espaces homogènes, Lecture Notes in Mathematics 242 (1971), Springer-Verlag, Berlin-New York.

[5] Coifman, R. and Weiss, G., Théorèmes sur les multiplicateurs de Fourier sur SU(2) et $\sum_{2}$, C. R. Acad. Sci. Paris Sér. A-B 271 (1970), pp A928-A930.

[6] Coifman, R. and Weiss, G., Multiplier transformations of functions on $\mathrm{SU}(2)$ and $\sum_{2}$, Collection of articles dedicated to Alberto González Domínguez on his sixty-fifth birthday, Rev. Un. Mat. Argentina, 25 (1970/71), pp 145-166.

[7] De Michele, L. and Mauceri, G., $L^{p}$ multipliers on the Heisenberg group, Michigan Math. J. 26 (1979), No 3, pp 361-371.

[8] Dixmier, J., von Neumann algebras, 27, Translated from the second French edition by F. Jellett, NorthHolland Publishing Co., Amsterdam-New York, 1981.

[9] Fischer, V., Intrinsic pseudo-differential calculi on any compact Lie group, J. Funct. Anal. 268 (2015), 11, pp 3404-3477.

[10] Fischer, V. and Ruzhansky, M., Fourier multipliers on graded Lie groups, preprint arXiv: 1411.6950.

[11] Hall, Brian, Lie groups, Lie algebras, and representations, Graduate Texts in Mathematics, Vol. 222, second edition, 2015.

[12] Hörmander, L., Estimates for translation invariant operators in $L^{p}$ spaces, Acta Math. 104 (1960), pp 93-140.

[13] Junge, M. and Mei, T. and Parcet, J., Smooth Fourier multipliers on group von Neumann algebras, Geom. Funct. Anal., 24, (2014), No 6, pp 1913-1980.

[14] Knapp, A., Lie groups beyond an introduction, Progress in Mathematics 140 (1996), Birkhäuser Boston, Inc., Boston, MA.

[15] Larsen, R., An introduction to the theory of multipliers, Die Grundlehren der mathematischen Wissenschaften, Band 175 (1971), Springer-Verlag, New York-Heidelberg.

[16] Marcinkiewicz, J., Sur les multiplicateurs des séries de Fourier, Studia Math. 8 (1939), pp 78-91. 
[17] Mauceri, G. and Meda, S., Vector-valued multipliers on stratified groups, Rev. Mat. Iberoamericana 6 (1990), No 3-4, pp 141-154.

[18] Mihlin, S. G., On the multipliers of Fourier integrals, Dokl. Akad. Nauk SSSR (N.S.) 109 (1956), pp 701-703.

[19] Rubin, R., Multipliers on the rigid motions of the plane and their relations to multipliers on direct products, Proc. Amer. Math. Soc. 59 (1976), No 1, pp 89-98.

[20] Ruzhansky, M. and Turunen, V., Pseudo-differential operators and symmetries, Pseudo-Differential Operators. Theory and Applications 2 (2010), Background analysis and advanced topics, Birkhäuser Verlag, Basel.

[21] Ruzhansky, M. and Wirth, J., $L^{p}$ Fourier multipliers on compact Lie groups, Math. Z. 280 (2015), 3-4, pp 621-642.

[22] Seeger, A. and Sogge, C. D., On the boundedness of functions of (pseudo-) differential operators on compact manifolds, Duke Math. J. 59 (1989), No 3, pp 709-736.

[23] Seeger, A. and Sogge, C. D. and Stein, E., Regularity properties of Fourier integral operators, Ann. of Math. (2) 134 (1991), No 2, pp 231-251,

[24] Sikora, A. and Wright, J., Imaginary powers of Laplace operators, Proc. Amer. Math. Soc. 129 (2001), No 6, pp 1745-1754 (electronic).

[25] Stanton, R., Mean convergence of Fourier series on compact Lie groups, Trans. Amer. Math. Soc. 218 (1976), pp 61-87.

[26] Stein, E., Singular integrals and differentiability properties of functions, Princeton Mathematical Series 30 (1970), Princeton University Press, Princeton, N.J.

[27] Stein, E., Topics in harmonic analysis related to the Littlewood-Paley theory, Annals of Mathematics Studies 63 (1970), Princeton University Press, Princeton, N.J.; University of Tokyo Press, Tokyo.

[28] Strichartz, R., Multiplier transformations on compact Lie groups and algebras, Trans. Amer. Math. Soc. 193 (1974), pp 99-110.

[29] Varopoulos, N. and Saloff-Coste, L. and Coulhon, T., Analysis and Geometry on Groups, Cambridge Tracts in Mathematics 100 (1992), Cambridge Univ. Press, Cambridge.

[30] Vretare, L., On $L_{p}$ Fourier multipliers on a compact Lie-group, Math. Scand. 35 (1974), pp 49-55.

[31] Weiss, N., $L^{p}$ estimates for bi-invariant operators on compact Lie groups, Amer. J. Math. 94 (1972), pp 103-118.

[32] Weiss, N., A multiplier theorem for SU(n), Proc. Amer. Math. Soc. 59 (1976), No 2, pp 366-370.

Department of Mathematical Sciences, University of Bath, Claverton Down, Bath Ba2 7AY, UNITED KINGDOM

E-mail address: v.c.m.fischer@bath.ac.uk 